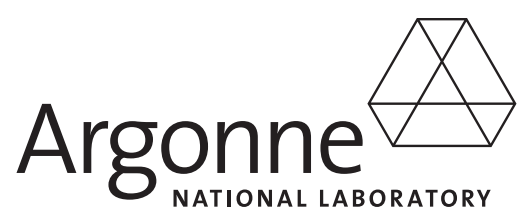

\title{
Control System Options and Strategies for Supercritical CO2 Cycles
}

Nuclear Engineering Division 


\begin{abstract}
About Argonne National Laboratory
Argonne is a U.S. Department of Energy laboratory managed by UChicago Argonne, LLC under contract DE-AC02-06CH11357. The Laboratory's main facility is outside Chicago, at 9700 South Cass Avenue, Argonne, Illinois 60439. For information about Argonne, see www.anl.gov.
\end{abstract}

\title{
Availability of This Report
}

This report is available, at no cost, at http://www.osti.gov/bridge. It is also available on paper to the U.S. Department of Energy and its contractors, for a processing fee, from:

U.S. Department of Energy

Office of Scientific and Technical Information

P.O. Box 62

Oak Ridge, TN 37831-0062

phone (865) 576-8401

fax (865) 576-5728

reports@adonis.osti.gov

\begin{abstract}
Disclaimer
This report was prepared as an account of work sponsored by an agency of the United States Government. Neither the United States Government nor any agency thereof, nor UChicago Argonne, LLC, nor any of their employees or officers, makes any warranty, express or implied, or assumes any legal liability or responsibility for the accuracy, completeness, or usefulness of any information, apparatus, product, or process disclosed, or represents that its use would not infringe privately owned rights. Reference herein to any specific commercial product, process, or service by trade name, trademark, manufacturer, or otherwise, does not necessarily constitute or imply its endorsement, recommendation, or favoring by the United States Government or any agency thereof. The views and opinions of document authors expressed herein do not necessarily state or reflect those of the United States Government or any agency thereof, Argonne National Laboratory, or UChicago Argonne, LLC.
\end{abstract}




\section{Control System Options and Strategies for Supercritical CO2 Cycles}

by

A. Moisseytsev, K. P. Kulesza* and J. J. Sienicki

Nuclear Engineering Division, Argonne National Laboratory

*Oregon State University

August 31, 2006 



\section{Table of Contents}

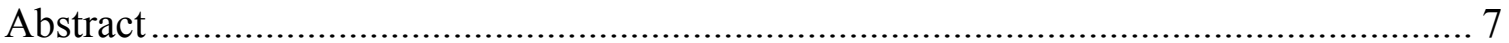

1. $\quad \mathrm{S}-\mathrm{CO}_{2}$ Brayton Cycle Dynamic Control .......................................................... 9

1.1. Justification of the Need for Plant Dynamics Code Development .....................9

1.2. Supercritical Carbon Dioxide Brayton Cycle Energy Converter

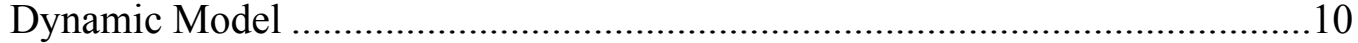

1.2.1. Heat Exchangers .......................................................................... 13

1.2.2. Pipes .............................................................................................. 14

1.2.3. Flow Split and Merge Points and Reversed Flow................................... 14

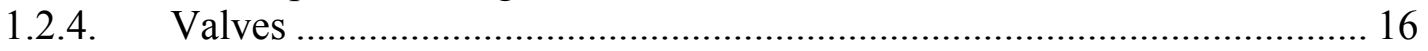

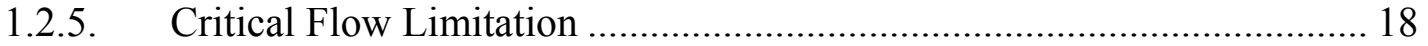

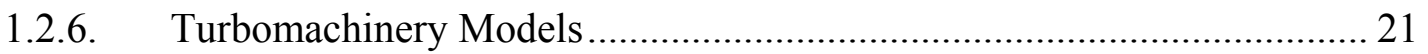

1.2.6.1 Compressor Off-Design Performance Model .................................... 22

1.2.6.2 Turbine Models........................................................................... 26

1.2.6.3 Turbomachinery in the Plant Dynamics Model ................................ 27

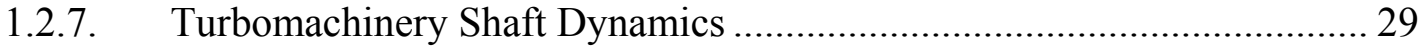

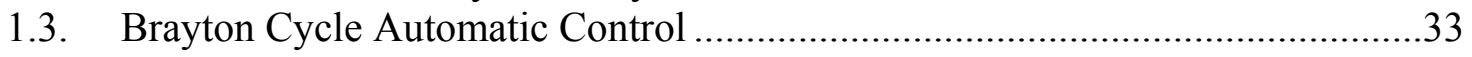

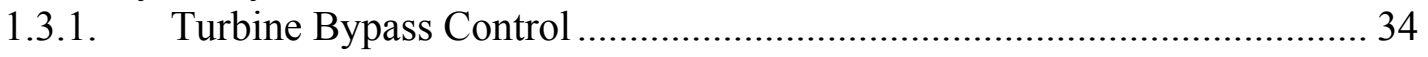

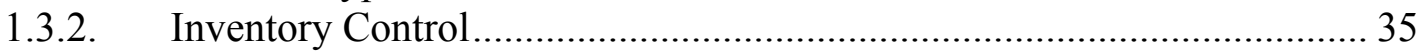

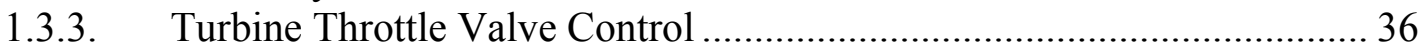

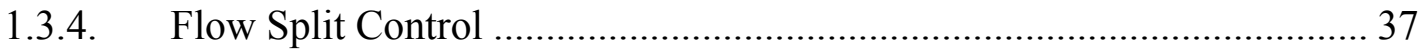

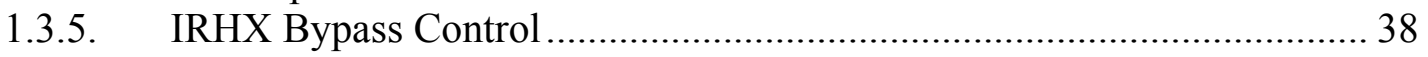

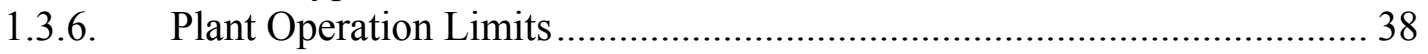

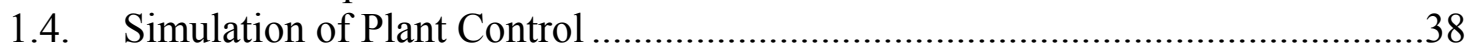

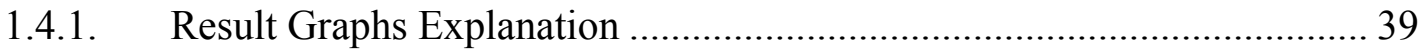

1.4.2. Full Power Operation - Steady-State and Stability Test ........................ 41

1.4.3. Plant Response to Step Changes in Load............................................... 42

1.4.4. Plant Response to Linear Changes in Load ........................................... 42

1.5. Summary and Proposed Future Developments .............................................79

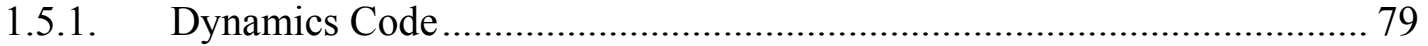

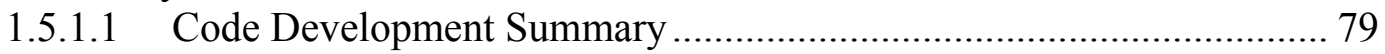

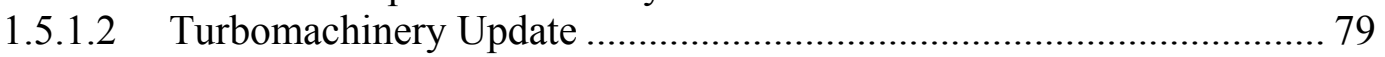

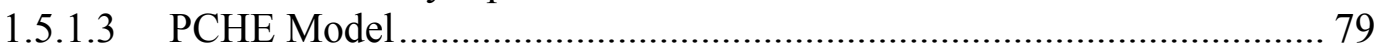

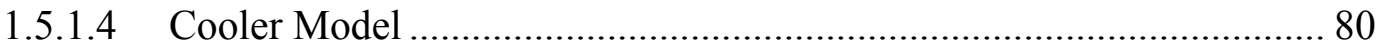

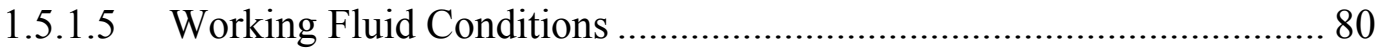

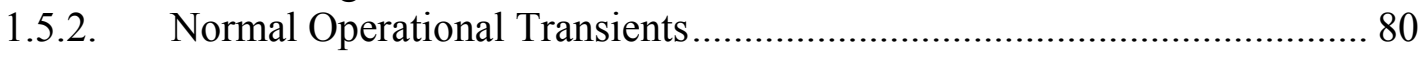

1.5.2.1 Summary of Normal Operation Analysis ....................................... 80

1.5.2.2 Startup and Shutdown, Decay Heat Removal................................... 81

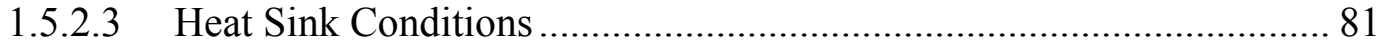

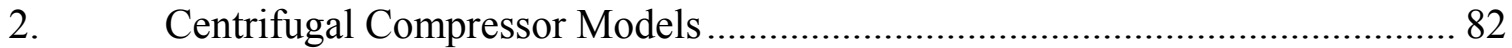

2.1. Centrifugal Compressor Design and Performance Models .............................82

2.2. Implementation of the Centrifugal Compressor Models .................................87

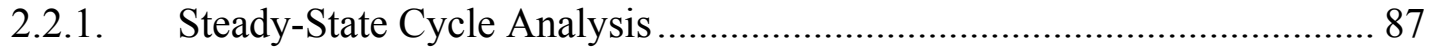

2.2.2. Off-Design Performance Analysis ................................................... 88

2.2.2.1 Compressor Performance Maps .................................................... 88 
2.2.2.2 Turbine Bypass Control …………………..................................... 90

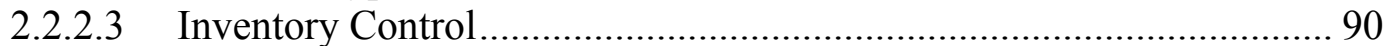

2.2.2.4 Turbine Inlet Valve Control............................................................... 96

2.2.2.5 Summary of Load Following Analysis for Centrifugal Compressors .. 96

3. Radial Inflow Turbine Analysis............................................................... 98

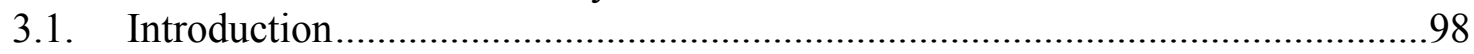

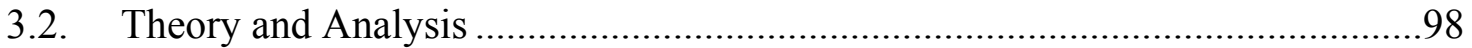

3.2.1. Boundary Layer Loss Coefficient...................................................... 99

3.2.2. Volute Analysis......................................................................... 100

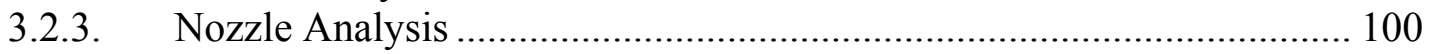

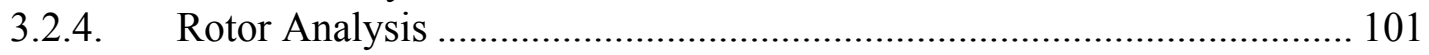

4. Turbomachinery Model Validation........................................................... 105

4.1. Compressor Performance Model Validation ...................................................105

4.2. Summary of Compressor Model Validation ...................................................109

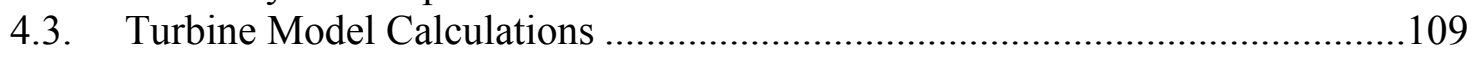

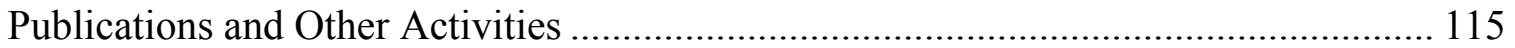

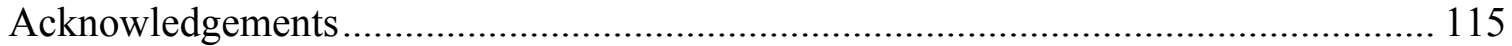

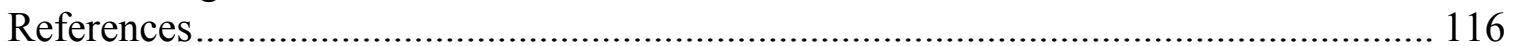




\section{List of Figures}

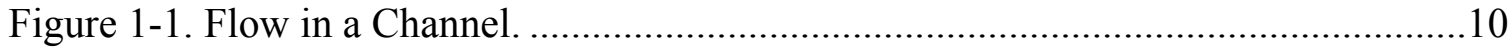

Figure 1-2. Regions for Temperature, Density, and Flow Rate Calculations....................12

Figure 1-3. Plant Dynamics Model Nodes...................................................................15

Figure 1-4. Flow Through a Valve Opening...............................................................18

Figure 1-5. Example of Compressor No. 1 Performance Map. ......................................25

Figure 1-6. Example of Compressor No. 2 Performance Map. ......................................25

Figure 1-7. Example of Turbine Performance Map........................................................27

Figure 1-8. Example of Compressor No. 1 Performance Map. ......................................29

Figure 1-9. Compressor Stage for Moment of Inertia Calculations..................................31

Figure 1-10. STAR-LM S- $\mathrm{CO}_{2}$ Brayton Cycle Layout and Control Mechanisms............33

Figure 1-11. Operation at Full Power. ................................................................43

Figure 1-12. 1\% Step Decrease in Load. ...................................................................49

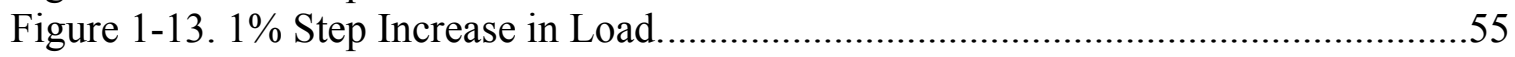

Figure 1-14. 10\% Step Decrease in Load. ..................................................................61

Figure 1-15. 50\% Linear Decrease in Load with Return to Full Load. ...........................67

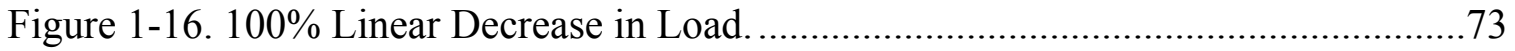

Figure 2-1. Illustration of a Two-Stage Centrifugal Compressor Design.........................83

Figure 2-2. Comparison of the Performance Maps for Axial and Centrifugal S- $\mathrm{CO}_{2}$

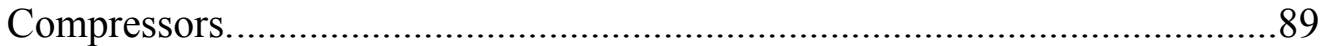

Figure 2-3. Turbine Bypass Control with Centrifugal Compressors. ...............................91

Figure 2-4. Inventory Control with Centrifugal Compressors.......................................93

Figure 2-5. Inventory Accumulation in Tank and Tank Pressure..................................95

Figure 2-6. Turbine Inlet Valve Control with Centrifugal Compressors..........................97

Figure 4-1. Comparison of Compressor Experiment Data with Model Calculations......107

Figure 4-2. Comparison of Compressor Experiment Data with Model Calculations with Flow Rate Modified within Uncertainty Range..................................108

Figure 4-3. Prediction of Radial Inflow Turbine Model for Pressure Ratio versus Mass Flow Rate for Pure Nitrogen at Turbine Inlet Pressure (TIP) of 199

$\mathrm{kPa}$ and Turbine Inlet Temperature (TIT) of $700 \mathrm{~K}$....

Figure 4-4. Prediction of Radial Inflow Turbine Model for Pressure Ratio versus Mass Flow Rate for Pure Nitrogen at Turbine Inlet Pressure (TIP) of $207.5 \mathrm{kPa}$ and Turbine Inlet Temperature (TIT) of $700 \mathrm{~K}$.

Figure 4-5. Prediction of Radial Inflow Turbine Model for Pressure Ratio versus Mass Flow Rate for Pure Nitrogen at Turbine Inlet Pressure (TIP) of $216.5 \mathrm{kPa}$ and Turbine Inlet Temperature (TIT) of $700 \mathrm{~K}$.

Figure 4-6. Prediction of Radial Inflow Turbine Model for Pressure Ratio versus Mass Flow Rate for Pure Nitrogen at Turbine Inlet Pressure (TIP) of $225.5 \mathrm{kPa}$ and Turbine Inlet Temperature (TIT) of $700 \mathrm{~K}$.

Figure 4-7. Prediction of Radial Inflow Turbine Model for Efficiency (Total-toStatic) versus Mass Flow Rate for Pure Nitrogen at Turbine Inlet Pressure (TIP) of $199 \mathrm{kPa}$ and Turbine Inlet Temperature (TIT) of $700 \mathrm{~K}$.

Figure 4-8. Prediction of Radial Inflow Turbine Model for Efficiency (Total-toStatic) versus Mass Flow Rate for Pure Nitrogen at Turbine Inlet 
Pressure (TIP) of $207.5 \mathrm{kPa}$ and Turbine Inlet Temperature (TIT) of 700

$\mathrm{K}$

Figure 4-9. Prediction of Radial Inflow Turbine Model for Efficiency (Total-toStatic) versus Mass Flow Rate for Pure Nitrogen at Turbine Inlet Pressure (TIP) of $216.5 \mathrm{kPa}$ and Turbine Inlet Temperature (TIT) of 700 $\mathrm{K}$.

Figure 4-10. Prediction of Radial Inflow Turbine Model for Efficiency (Total-toStatic) versus Mass Flow Rate for Pure Nitrogen at Turbine Inlet Pressure (TIP) of $225.5 \mathrm{kPa}$ and Turbine Inlet Temperature (TIT) of 700

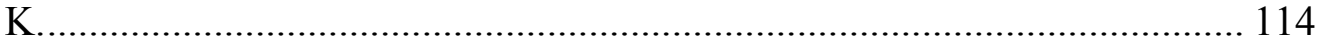




\section{List of Tables}

Table 1-1. Moments of Inertia 32

Table 2-1. Comparison of Axial and Centrifugal Compressors .....................................87

Table 4-1. Compressor Design Parameters for the Performance Model ........................106

Table 4-2. Compressor Experiment Data for Pure Nitrogen ........................................ 107

Table 4-3. Radial Inflow Turbine Model Calculations for Pure Nitrogen at Turbine

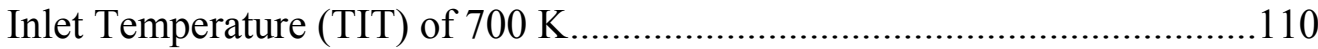




\title{
Control System Options and Strategies for Supercritical $\mathrm{CO}_{2}$ Cycles
}

\author{
by \\ Anton Moisseytsev, Konrad P. Kulesza, ${ }^{1}$ and James J. Sienicki
}

\begin{abstract}
The Supercritical Carbon Dioxide $\left(\mathrm{S}-\mathrm{CO}_{2}\right)$ Brayton Cycle is a promising alternative to Rankine steam cycle and recuperated gas Brayton cycle energy converters for use with Sodium-Cooled Fast Reactors (SFRs), Lead-Cooled Fast Reactors (LFRs), as well as other advanced reactor concepts. The $\mathrm{S}-\mathrm{CO}_{2}$ Brayton Cycle offers higher plant efficiencies than Rankine or recuperated gas Brayton cycles operating at the same liquid metal reactor core outlet temperatures as well as reduced costs or size of key components especially the turbomachinery. A new Plant Dynamics Computer Code has been developed at Argonne National Laboratory for simulation of a $\mathrm{S}-\mathrm{CO}_{2}$ Brayton Cycle energy converter coupled to an autonomous load following liquid metal-cooled fast reactor. The Plant Dynamics code has been applied to investigate the effectiveness of a control strategy for the S-CO $\mathrm{CO}_{2}$ Brayton Cycle for the STAR-LM $181 \mathrm{MWe}(400 \mathrm{MWt})$ Lead-Cooled Fast Reactor. The strategy, which involves a combination of control mechanisms, is found to be effective for controlling the $\mathrm{S}_{-} \mathrm{CO}_{2}$ Brayton Cycle over the complete operating range from 0 to $100 \%$ load for a representative set of transient load changes. While the system dynamic analysis of control strategy performance for STAR$\mathrm{LM}$ is carried out for a $\mathrm{S}-\mathrm{CO}_{2}$ Brayton Cycle energy converter incorporating an axial flow turbine and compressors, investigations of the $\mathrm{S}_{-} \mathrm{CO}_{2}$ Brayton Cycle have identified benefits from the use of centrifugal compressors which offer a wider operating range,

${ }^{1}$ Oregon State University
\end{abstract}


greater stability near the critical point, and potentially further cost reductions due to fewer stages than axial flow compressors. Models have been developed at Argonne for the conceptual design and performance analysis of centrifugal compressors for use in the S$\mathrm{CO}_{2}$ Brayton Cycle. Steady state calculations demonstrate the wider operating range of centrifugal compressors versus axial compressors installed in a $\mathrm{S}_{-} \mathrm{CO}_{2}$ Brayton Cycle as well as the benefits in expanding the range over which individual control mechanisms are effective for cycle control. However, a combination of mechanisms is still required for control of the $\mathrm{S}-\mathrm{CO}_{2}$ Brayton Cycle between 0 and $100 \%$ load. An effort is underway to partially validate the Argonne models and codes by means of comparison with data from tests carried out using the small-scale Sandia Brayton Loop (SBL) recuperated gas closed Brayton cycle facility. The centrifugal compressor model has been compared with data from the SBL operating with nitrogen gas and good agreement is obtained between calculations and the measured data for the compressor outlet pressure versus flow rate, although it is necessary to assume values for certain model parameters which require information about the configuration or dimensions of the compressor components that is unavailable. Unfortunately, the compressor efficiency cannot be compared with experiment data due to the lack of outlet temperature data. A radial inflow turbine model has been developed to enable further comparison of calculations with data from the SBL which incorporates both a radial inflow turbine as well as a radial compressor. Preliminary calculations of pressure ratio and efficiency versus flow rate have been carried out using the radial inflow turbine model. 


\section{1. $\mathrm{S}-\mathrm{CO}_{2}$ Brayton Cycle Dynamic Control}

The previous status report for FY 2005 [1] summarized the development of a S$\mathrm{CO}_{2}$ Brayton cycle control strategy using steady-state load following computer code. This year, control of the cycle was investigated with a detailed system dynamics code. A special Plant Dynamics Computer Code has been developed to address specific dynamic features of the $\mathrm{S}-\mathrm{CO}_{2}$ Brayton cycle. The code was developed for the $\mathrm{S}-\mathrm{CO}_{2}$ Brayton cycle coupled to the Secure Transportable Autonomous Reactor with Liquid Metal coolant (STAR-LM) Lead-Cooled Fast Reactor (LFR).

The initial development of the Plant Dynamics Code was carried out for the case of an axial-flow turbine and compressors. The development of models for radial-flow (i.e., centrifugal) compressors and turbine is described in Section 2.

\subsection{Justification of the Need for Plant Dynamics Code Development}

The $\mathrm{S}-\mathrm{CO}_{2}$ Brayton cycle has several specific features which limit the applicability of the existing computer codes. First, the main feature of the cycle is that it takes advantage of sharp $\mathrm{CO}_{2}$ properties changes near the critical point. Therefore, accurate calculations near the critical point are very important to reliably calculate the cycle performance. At the same time, the majority of the Brayton cycle analysis codes use an ideal-gas approximation for turbomachinery design and performance. Since this approximation is not applicable to the $\mathrm{S}-\mathrm{CO}_{2}$ Brayton, those codes could not be applied here. Also, for the system that is analyzed in which the cycle is used as a power converter for a LFR, the cycle performance affects the reactor behavior, so the Brayton cycle model with accurate representations of the $\mathrm{CO}_{2}$ properties must be tightly coupled to the fastreactor systems dynamics model.

Other incentives for new code development are:

- to have a modular code where parts and models can be changed and replaced with other models for the design and applicability to other reactor system types; and

- to develop a code which has a sufficiently short running time such that many simulations can be performed and investigations of the effects of different changes in design on the transient performance can be carried out (the existing multi-dimensional codes are too slow for such investigations).

For the reasons described above, it was decided to develop a special dynamics code rather than modify and apply an existing code to this system. Still, the code allows for implementation of additional external models, if it would be helpful. For example, the current version of the code uses the point kinetics subroutines from the SAS4A/SASSYS1 code to solve for the reactor power. 


\subsection{Supercritical Carbon Dioxide Brayton Cycle Energy Converter Dynamic Model}

Since the $\mathrm{CO}_{2}$ is a compressible fluid, the development of compressible flow dynamical equations is needed to correctly model the $\mathrm{CO}_{2}$ flow and Brayton cycle.

Consider a flow of a compressible fluid in a heated channel (Figure 1-1).

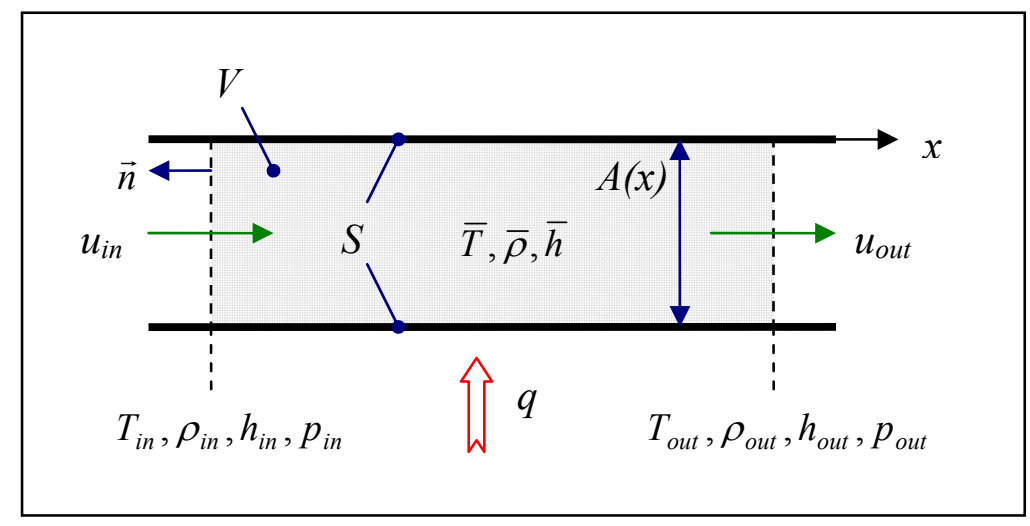

Figure 1-1. Flow in a Channel.

The dynamical behavior of a compressible flow in a channel is described by mass (1-1), momentum (1-2), and energy (1-3) conservation equations [2]:

$$
\begin{aligned}
& \frac{\partial}{\partial t} \int_{V} \rho d V+\int_{S} \rho \vec{u} \cdot \vec{n} d S=0 \\
& \frac{\partial}{\partial t} \int_{V} \rho \vec{u} d V+\int_{S} \rho \vec{u} \vec{u} \cdot \vec{n} d S=\int_{V} \rho \vec{g} d V+\int_{S} \vec{n} \cdot \tau d S \\
& \frac{\partial}{\partial t} \int_{V}\left(\rho e+\frac{1}{2} \rho(\vec{u})^{2}-\rho \vec{g} \cdot \vec{x}\right) d V+\int_{S}\left(\rho e+\frac{1}{2} \rho(\vec{u})^{2}-\rho \vec{g} \cdot \vec{x}\right) \vec{u} \cdot \vec{n} d S \\
& =-\int_{S} \vec{q} \cdot \vec{n} d S+\int_{S} \vec{n} \cdot(\tau \cdot \vec{u}) d S
\end{aligned}
$$

where $S$ = boundary of the volume $V$,

$\vec{n}=$ normal to the surface $S$,

$\rho=$ density,

$\vec{u}=$ velocity,

$\vec{g}=$ gravitational acceleration,

$\tau=$ stress tensor,

$e=$ internal energy,

$\vec{q}=$ heat flux. 
The following assumptions are made to simplify the dynamics equations.

- Ignore gravitational effects.

- Stress is represented by the friction at the channel wall only. This assumption means that the form losses are neglected compared to the frictional losses, which is expected to be true for the $\mathrm{S}-\mathrm{CO}_{2}$ Brayton cycle. Also the equations are to be applied to a channel of constant cross-sectional area $(A=$ const $)$. The treatment of the flow through a valve - where form losses are important - is described separately below.

- Ignore acceleration pressure drop.

- Ignore energy loss due to friction.

- The total energy is represented by the specific enthalpy. This assumption means ignoring the kinetic energy of the flow. Since the kinetic energy is important in turbomachinery, this model is to be applied to the flow in heat exchangers and piping; a separate model is used for turbomachinery.

- The flow in the volume is characterized by mean properties and flow rates.

Under these assumptions, the conservation equations can be expressed in the following forms, respectively [2]:

$$
\begin{aligned}
& \frac{\partial \bar{\rho}}{\partial t}=\frac{\dot{m}_{\text {in }}-\dot{m}_{\text {out }}}{A \Delta x} \\
& \frac{\partial \overline{\dot{m}}}{\partial t}=\left(p_{\text {in }}-p_{\text {out }}\right) \frac{A}{\Delta x}-2 \bar{f} \frac{\overline{\dot{m}}^{2}}{A \bar{\rho} D_{h}} \\
& \frac{\partial \bar{h}}{\partial t}=\frac{1}{\Delta x A \bar{\rho}}\left[\dot{m}_{\text {in }}\left(h_{\text {in }}-\bar{h}\right)-\dot{m}_{\text {out }}\left(h_{\text {out }}-\bar{h}\right)+q^{\prime} \Delta x\right]
\end{aligned}
$$

To apply Equations (1-4) through (1-6) in a computer code, they should be expressed in three variables only - the flow rate and two variables that define the fluid state. Since the $\mathrm{CO}_{2}$ properties are originally defined in terms of the temperature and density, selecting these variables would simplify the $\mathrm{CO}_{2}$ properties calculations and, therefore, increase the computational speed of the dynamics code. The other parameters (enthalpy and pressure) are expressed in terms of the temperature and density as following:

$$
\begin{aligned}
& \frac{\partial h}{\partial t}=\left(\frac{\partial h}{\partial T}\right)_{\rho} \frac{\partial T}{\partial t}+\left(\frac{\partial h}{\partial \rho}\right)_{T} \frac{\partial \rho}{\partial t}=h_{T} \frac{\partial T}{\partial t}+h_{\rho} \frac{\partial \rho}{\partial t} \\
& \frac{\partial p}{\partial t}=\left(\frac{\partial p}{\partial T}\right)_{\rho} \frac{\partial T}{\partial t}+\left(\frac{\partial p}{\partial \rho}\right)_{T} \frac{\partial \rho}{\partial t}=p_{T} \frac{\partial T}{\partial t}+p_{\rho} \frac{\partial \rho}{\partial t}
\end{aligned}
$$

The partial derivatives in Equations (1-7) and (1-8) are derived analytically from the known functions, $p=p(T, \rho), h=h(T, \rho)$. 
The $\mathrm{CO}_{2}$ conditions (temperature and density) are calculated at the region boundaries (inlet and outlet); the flow rate is calculated between the boundaries (Figure 1-1). In order to calculate the density at the boundary, Equation (1-4) is applied to the region consisting of two halves of the temperature regions from each side of the boundary (Figure 1-2).

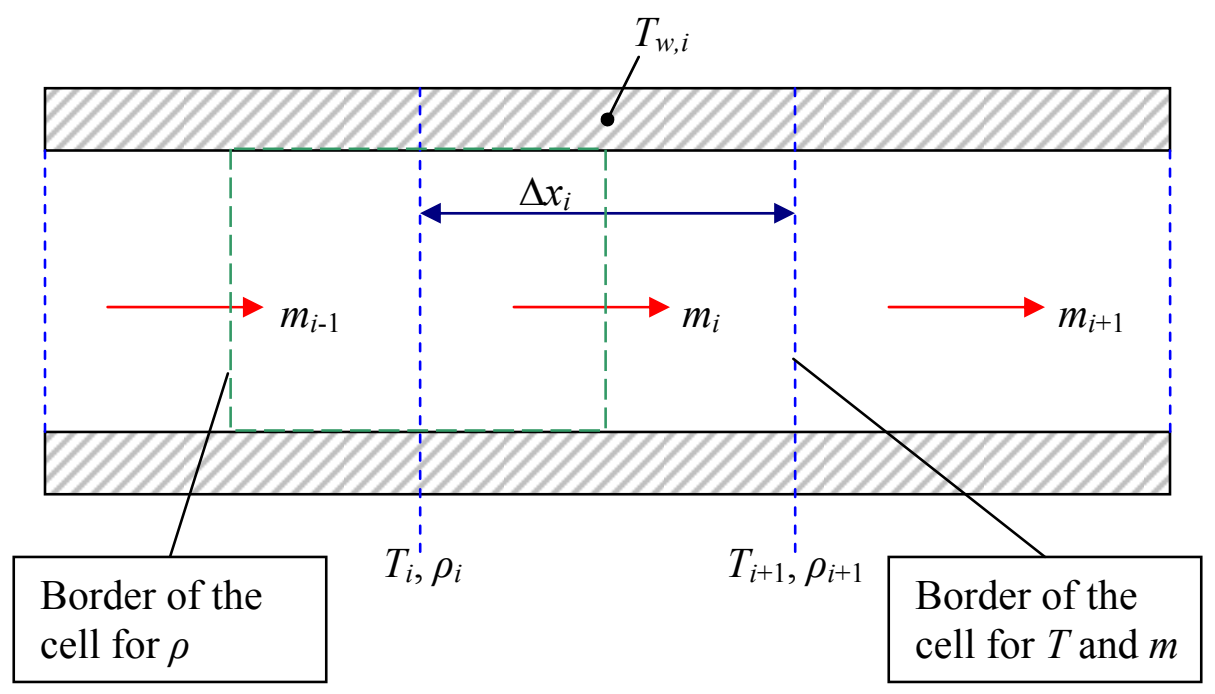

Figure 1-2. Regions for Temperature, Density, and Flow Rate Calculations.

$$
\frac{\partial \rho_{i}}{\partial t}=\frac{1}{A\left(\frac{\Delta x_{i}}{2}+\frac{\Delta x_{i-1}}{2}\right)}\left(\dot{m}_{i}-\dot{m}_{i-1}\right)
$$

The momentum Equation (1-5) applied to the region in Figure 1-2 gives

$$
\frac{\partial \dot{m}_{i}}{\partial t}=\frac{A}{\Delta x_{i}}\left(p_{i}-p_{i+1}\right)-\frac{2 f_{i} \Delta x_{i}}{M_{i} D_{h}} \dot{m}_{i}^{2}
$$

where

$$
M_{i}=A \bar{\rho} \Delta x_{i}=\mathrm{CO}_{2} \text { mass in region } i .
$$

To apply the energy Equation (1-6) to the temperature region in Figure 1-2, an assumption of perfect mixing is made. Under this assumption, the enthalpy of the flow leaving the region is the same as the average enthalpy in the region $\left(h_{\text {out }} \approx \bar{h}\right)$. The heat flux from the wall is defined by the temperature difference between the wall and average $\mathrm{CO}_{2}$ temperature and the thermal resistance between the wall and $\mathrm{CO}_{2}$ flow. The inlet flow rate is calculated as an average between the flow rates adjacent to the inlet boundary. 


$$
\frac{\partial h_{i+1}}{\partial t}=\frac{1}{M_{i}} \frac{\dot{m}_{i}+\dot{m}_{i-1}}{2}\left(h_{i}-h_{i+1}\right)+\frac{\Delta x_{i} N_{t}}{M_{i} \operatorname{res}_{w, C O_{2 i}}}\left(T_{w, i}-\frac{T_{i}+T_{i+1}}{2}\right)
$$

where

$N_{t}=$ total number of channels (tubes) for heat transfer,

res ${ }_{w, \mathrm{CO}_{2}}=$ thermal resistance between the wall and $\mathrm{CO}_{2}$.

All coefficients (like mass and thermal resistance) in Equations (1-9)-(1-11) are assumed to be constant during the time step. To calculate the other properties derivatives, Relationships (1-7) and (1-8) are used:

$$
\begin{aligned}
& \frac{\partial T_{i+1}}{\partial t}=\frac{1}{h_{T_{i+1}}}\left(\frac{\partial h_{i+1}}{\partial t}-h_{\rho_{i+1}} \frac{\partial \rho_{i+1}}{\partial t}\right) \\
& \frac{\partial p_{i+1}}{\partial t}=p_{T_{i+1}} \frac{\partial T_{i+1}}{\partial t}+p_{\rho_{i+1}} \frac{\partial \rho_{i+1}}{\partial t}
\end{aligned}
$$

\subsubsection{Heat Exchangers}

Equations (1-9)-(1-13) are readily applied to the flow in the heat exchangers. For the boundary nodes (HX inlet or outlet), the parameters of the connecting pipes ( $\Delta x$ and $\dot{m}$ ) are used in Equations (1-9) and (1-11), if the index is beyond the heat exchanger (e.g. $\dot{m}_{i-1}$ is equal to the flow rate in the inlet pipe for region $\left.i=1\right)$.

The region indexing is selected such that the border number increases in the direction of the hot flow ${ }^{1}\left(i=1\right.$ for the hot flow inlet). For the cold flow $\left(\mathrm{CO}_{2}\right.$ in IRHX, high pressure flows in the HTR and LTR), the indexing direction is opposite to the flow ( $i=1$ at the outlet).

The HX wall (metal) temperature is calculated based on the energy balance and average temperatures of hot and cold flows, as shown in Equation (1-14). The wall temperature is calculated between flow temperature nodes as indicated in Figure 1-2.

$$
\frac{\partial T_{w, i}}{\partial t}=\frac{\Delta x_{i} N_{t}}{M_{w, i} C p_{w, i}}\left[\frac{1}{r e s_{w, h_{i}}}\left(\frac{T_{h, i}+T_{h, i+1}}{2}-T_{w, i}\right)-\frac{1}{r e s_{w, c_{i}}}\left(T_{w, i}-\frac{T_{c, i}+T_{c, i+1}}{2}\right)\right]
$$

where

$M_{w, i}=A \rho_{w, i} \Delta x_{i}=$ metal mass in region $i$.

$r e s_{w, h}, r e s_{w, c}=$ thermal resistance between the wall and hot and cold flows, respectively.

\footnotetext{
${ }^{1}$ Hot and cold flows are defined based on steady-state conditions.
} 
Although the same approach as for the other heat exchangers could also be applied to the cooler, a simplified model is used for the following reasons. First, it is noticed that the time step required for the momentum Equation (1-10) decreases as $\mathrm{CO}_{2}$ conditions approach the critical point (due to increasing compressibility). So, implementing the same model for the cooler, even with a small number of regions, would result in significant increase in the computational time. Moreover, due to the strong properties variation near the critical point, a large number of the regions is required to accurately represent the conditions inside the cooler (In steady-state mode, 50 regions are used for a cooler while only 10-20 regions are used for the recuperators). This would further slow down the calculations. Therefore, it was decided to implement a simplified model for the cooler. Again, the same model as for the other heat exchangers could be easily applied to the cooler in the future, if the computational time is not an issue. The simplified cooler model assumes that the minimum (cooler-outlet) temperature in the cycle is always maintained at the steady-state value. Thus, the temperature at the cooler outlet is fixed. To calculate the densities at the cooler inlet and outlet, a one-region approach is applied to Equations (1-9) and (1-10) to calculate an average flow rate in the cooler. The cooler-average friction factor is calculated for steady-state conditions and is assumed to be constant during the transient.

\subsubsection{Pipes}

The equations derived above are applied to the flows in the pipes in the Brayton cycle with the following simplifications. First, it is assumed that the pipes are insulated so that there is no heat transfer term in Equation (1-11). Also, the frictional pressure loss inside of the pipes (Equation (1-10)) is neglected.

The indexing of the regions for the pipe is selected such that the temperature and density are calculated at each important point of the cycle, such as the HX, turbine and compressor inlets and outlets, flow merge and split points. The flow rates are calculated between these points. Figure 1-3 shows the selected nodes for whole system including the reactor and Brayton cycle. The squares show the points for the temperature and density; the circles show the flow rate positions. Some additional points for the flow rates are added for a convenience. For example, the flow rate at the HTR inlet, $\dot{m}_{5}$, is used to calculate the density, $\rho_{5}$, according to Equation (1-9) but is actually calculated as a flow rate in the first region in the HTR.

Equation (1-10) assumes that there is no pressure loss due to the form losses. The corrections to the flow rate through the pipes with valves are described below.

\subsubsection{Flow Split and Merge Points and Reversed Flow}

At the flow merge points (such as Point 2 in Figure 1-3) there is more than a single inlet flow. Therefore, Equation (1-9) should have more than one inlet flow term. Also, Equation (1-11) will have more than one term for the heat addition due to inlet flows. Similarly, Equation (1-9) should be modified to include more than one outlet flow for the flow split points (such as Point 23). Also, the volumes for Equation (1-9) would 
include three terms for the merge and split point. Therefore, Equations (1-9) and (1-11) are modified as follows.

Before the dynamics calculations, the volumes for each density point are calculated. These volumes would include two adjustment regions halves for the regular point and three halves for flow split/merge points. Then, in the dynamic calculations, the derivative of the density is calculated as a sum of all of the inlet flow rates minus the sum of all outlet flow rates divided by the volume.

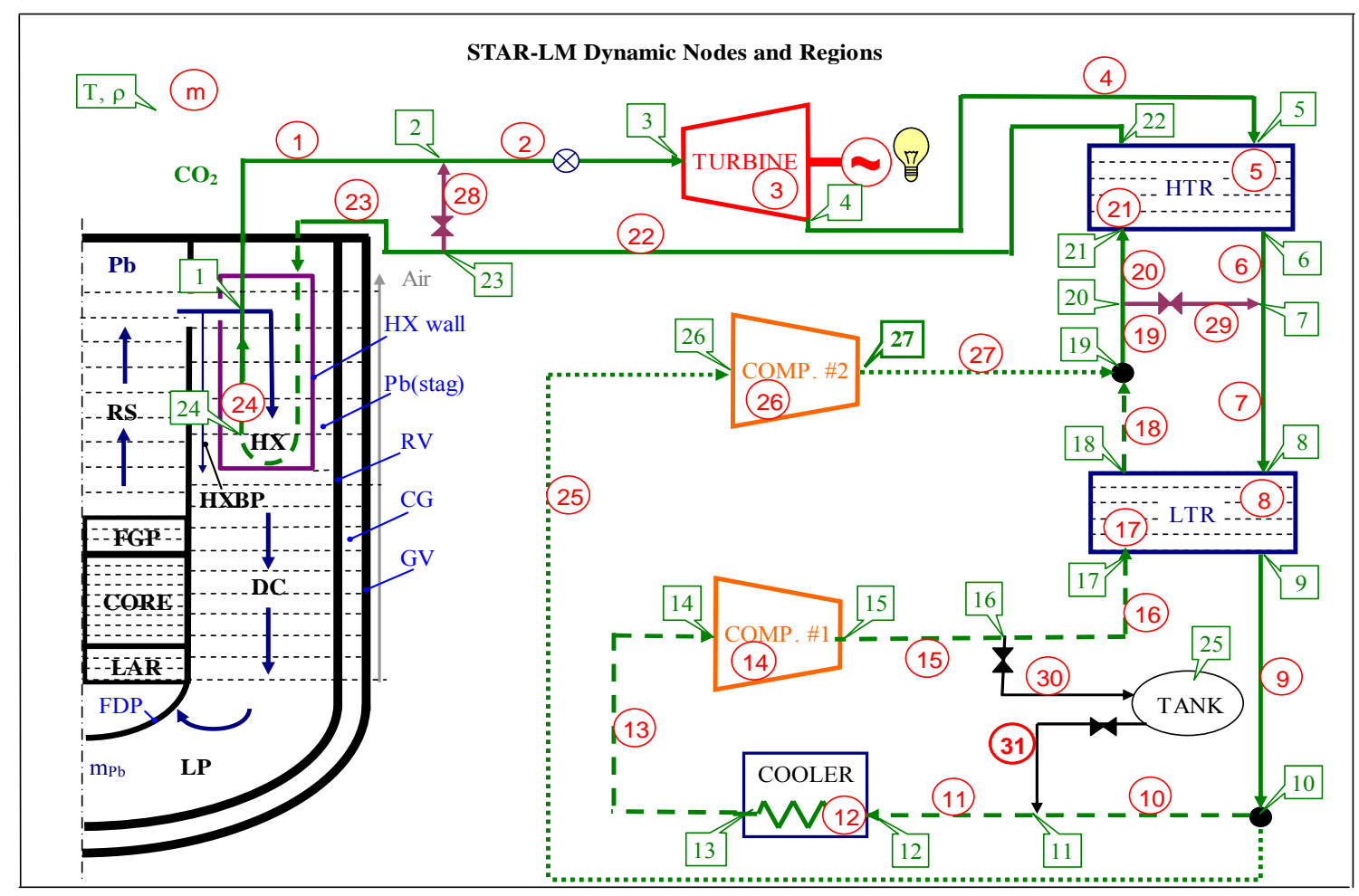

Figure 1-3. Plant Dynamics Model Nodes.

The same approach is used for Equation (1-11) to account for several inlet flows. This approach is combined with the treatment of negative flows.

A solution of the flow equation may result in a reversed flow, i.e. negative values of flow rates. The reversed flow would exchange the inlet and outlet points in Equations (1-9)-(1-11). To account for reversed flows and their effect on the equations, the following approach is used. Before the dynamics calculations, two arrays are generated which define inlet and outlet nodes for each flow rate in the system (including flows in the heat exchangers) under steady-state (positive flow) conditions. If, during a transient the flow is positive, then the outlet node for this flow as the same as at steady-state; if the flow is negative, then the outlet node is the steady-state inlet node. At the beginning of a time step, the density and enthalpy derivatives for each point are set to zero. Then, for each flow rate, the value of the density derivative is increased at the outlet node and decreased at the inlet node. Also, the value of the enthalpy derivative is increased for the outlet node. 


$$
\begin{aligned}
& \frac{\partial \rho_{i-\text { out }}}{\partial t}=\frac{\partial \rho_{i-\text { out }}}{\partial t}+\frac{1}{V_{\rho, i-\text { out }}} \dot{m}_{i} \\
& \frac{\partial \rho_{i-\text { in }}}{\partial t}=\frac{\partial \rho_{i-\text { in }}}{\partial t}-\frac{1}{V_{\rho, i-\text { in }}} \dot{m}_{i} \\
& \frac{\partial h_{i-\text { out }}}{\partial t}=\frac{\partial h_{i-\text { out }}}{\partial t}+\frac{1}{M_{i}}\left|\dot{m}_{\text {in }}\right|\left(h_{i-\text { in }}-h_{i-\text { out }}\right)+\frac{\Delta x_{i} N_{t}}{M_{i} \operatorname{res}_{w, C O_{2 i}}}\left(T_{w, i}-\frac{T_{i}+T_{i+1}}{2}\right)
\end{aligned}
$$

where

$i$-in and $i$-out $=$ indices for the inlet and outlet nodes for flow, $\dot{m}_{i}$, the heat transfer term is omitted for the pipes.

Similar to the momentum equation for the reactor coolant, the flow equation is modified to account for negative flow:

$$
\frac{\partial \dot{m}_{i}}{\partial t}=\frac{A}{\Delta x_{i}}\left(p_{i}-p_{i+1}\right)-\frac{2 f_{i} \Delta x_{i}}{M_{i} D_{h}} \dot{m}_{i}\left|\dot{m}_{i}\right|
$$

\subsubsection{Valves}

Valves are used in the Brayton cycle for control purposes. There are six valves programmed in the code (with the corresponding flow rates in Figure 1-3 given in parentheses): IRHX bypass valve (28), turbine throttle valve (2), turbine bypass valve (29), Compressor No. 2 throttle valve (25), and inventory tank inlet (30) and outlet (31) valves. The valves are used to regulate flow by adding a resistance to the flow. In order to model the hydraulic resistance presented by a valve, a momentum Equation (1-18) is modified to account for the pressure losses in the valve. The valve is simulated as a sudden flow area contraction followed by a sudden flow area expansion. The total pressure loss is represented by the sum of the form losses from contraction and expansion:

$$
\Delta p_{v}=\frac{1}{2} K_{\text {contr }} \rho u_{\text {small }}^{2}+\frac{1}{2} K_{\text {exp }} \rho u_{\text {small }}^{2}
$$

where

$$
\begin{aligned}
& K_{\text {contr }}=\frac{1}{2}\left(1-\frac{A_{\text {open }}}{A_{\text {total }}}\right)=\text { contraction loss coefficient, } \\
& K_{\text {exp }}=\left(1-\frac{A_{\text {open }}}{A_{\text {total }}}\right)^{2}=\text { expansion loss coefficient, } \\
& A_{\text {open }}=\text { flow area open to the flow (flow area inside a valve), } \\
& A_{\text {total }}=\text { total flow area (flow area outside a valve), }
\end{aligned}
$$


$\rho=$ density,

$u_{\text {small }}=$ flow speed at the opening.

The following variables are introduced to simplify Equation (1-19).

- The ratio of the open area to the total area is defined as the valve open area fraction. The fraction is either user-defined or a result of the control calculations.

$$
f_{\text {open }}=\frac{A_{\text {open }}}{A_{\text {total }}}
$$

- Two loss coefficients are combined to form a valve loss coefficient:

$$
\begin{aligned}
K_{v} & =K_{\text {contr }}+K_{\text {exp }}=\frac{1}{2}\left(1-f_{\text {open }}\right)+\left(1-f_{\text {open }}\right)^{2}=\frac{1}{2}-\frac{f_{\text {open }}}{2}+1-2 f_{\text {open }}+f_{\text {open }}^{2} \\
& =1 \frac{1}{2}-1 \frac{1}{2} f_{\text {open }}-f_{\text {open }}+f_{\text {open }}^{2}=1 \frac{1}{2}\left(1-f_{\text {open }}\right)-f_{\text {open }}\left(1-f_{\text {open }}\right) \\
& =\left(1.5-f_{\text {open }}\right)\left(1-f_{\text {open }}\right)
\end{aligned}
$$

- The flow speed is calculated based on the continuity equation:

$$
u_{\text {small }}=\frac{\dot{m}}{\rho A_{\text {open }}}
$$

- The density is calculated as the average density between inlet and outlet conditions:

$$
\rho=\frac{\rho_{\text {in }}+\rho_{\text {out }}}{2}
$$

Using the above definitions, the pressure drop across the valve becomes:

$$
\Delta p_{v}=\frac{1}{2} K_{v} \frac{1}{\rho A_{\text {open }}^{2}} \dot{m}^{2}=\frac{1}{2} K_{v} \frac{1}{\rho f_{\text {open }}^{2} A_{\text {total }}^{2}} \dot{m}^{2}
$$

The open area fraction in the denominator is added to the valve loss coefficient to collect valve openings in one function:

$$
K_{v}^{\prime}=\frac{K_{v}}{f_{\text {open }}^{2}}=\frac{\left(1.5-f_{\text {open }}\right)\left(1-f_{\text {open }}\right)}{f_{\text {open }}^{2}}
$$


After the derivatives for all flow rates in the cycle are calculated using Equation (1-18), the derivatives of the flow rates through valves are corrected to add the valve resistance (Equation (1-24) is corrected to allow for a negative flow):

$$
\frac{\partial \dot{m}_{i-v}}{\partial t}=\frac{\partial \dot{m}_{i-v}}{\partial t}-\frac{A_{i-v}}{\Delta x_{i-v}} \frac{K_{v}^{\prime}}{2 \rho A_{i-v}^{2}} \dot{m}_{i-v}\left|\dot{m}_{i-v}\right|
$$

where

$i-v=\{2,25,28,29,30,31\}=$ indices for the valves locations.

\subsubsection{Critical Flow Limitation}

According to the momentum Equation (1-18), the flow between two points is determined by the pressures at those points. Pressure waves travel through a fluid at the sound speed. If the flow rate is high enough that the local speed reaches sonic speed, the conditions downstream of this point could not be communicated upstream any more. Therefore, the flow rate cannot increase even if outlet pressure decreases. This condition is called critical flow.

Critical flow occurs when the local speed reaches the speed of sound. According to the continuity equation, speed is reciprocal to the flow area, so that the maximum speed is achieved at the minimum flow area. In the cycle, the minimum flow areas occur at a valve opening. Therefore, it is expected that the flow in a valve could reach critical flow. The flow could not be increased beyond the critical flow no matter how low the outlet pressure is, even if Equation (1-18) allows for that. So, the critical flow rate should be calculated for the conditions of each valve and the limitation of the critical flow should be applied to the flow.

Consider flow through a valve (Figure 1-4). The conditions of the flow before the valve (Point 1) are given. At critical flow, the flow speed in the opening is equal to the sound speed defined for local properties.

$$
u_{0}=V_{s, 0} \equiv V_{s}\left(h_{0}, s_{0}\right)
$$

where $V_{s}=$ sound speed.

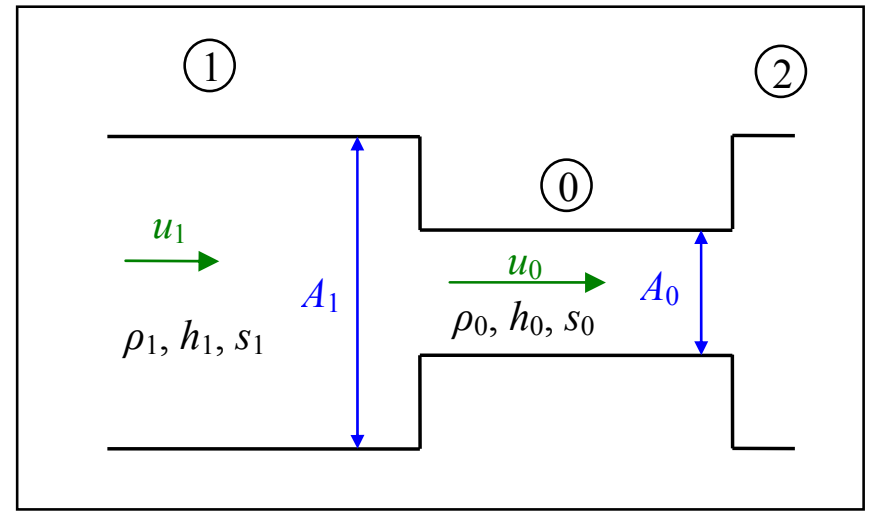

Figure 1-4. Flow Through a Valve Opening. 
The critical flow rate is defined from the continuity equation.

$$
\dot{m}_{c}=\rho_{0} u_{0} A_{0}=\rho_{0} u_{0} A_{1} f_{\text {open }}
$$

where

$$
f_{\text {open }}=\frac{A_{0}}{A_{1}}=\text { valve open area fraction. }
$$

To find the conditions at the opening and the local sound speed an iterative process is applied. It is assumed that the flow through the valve is isentropic, i.e. $s_{0}=S_{1}$. Suppose that the density and enthalpy at the opening are known (for the first approximation, the inlet parameters could be taken). Then the continuity equation defines the flow speed before the valve:

$$
u_{1}=\frac{\dot{m}_{c}}{\rho_{1} A_{1}}
$$

The enthalpy at the opening is found from the total energy conservation equation:

$$
\begin{aligned}
& h_{1}+\frac{u_{1}^{2}}{2}=h_{0}+\frac{u_{0}^{2}}{2} \\
& h_{0}=h_{1}+\frac{u_{1}^{2}}{2}-\frac{u_{0}^{2}}{2}=h_{1}+\frac{1}{2}\left(\frac{\dot{m}_{c}}{\rho_{1} A_{1}}\right)^{2}-\frac{V_{s, 0}^{2}}{2}=h_{1}+\frac{1}{2}\left(\frac{\rho_{0} A_{0} V_{s, 0}}{\rho_{1} A_{1}}\right)^{2}-\frac{V_{s, 0}^{2}}{2} \\
& h_{0}=h_{1}+\frac{V_{s, 0}^{2}}{2}\left[\left(\frac{\rho_{0}}{\rho_{1}}\right)^{2} f_{\text {open }}^{2}-1\right]
\end{aligned}
$$

When the enthalpy and entropy at the opening are known, the density and the sound speed are recalculated based on the $\mathrm{CO}_{2}$ properties.

$$
\begin{gathered}
\rho_{0}=\rho\left(s_{0}, h_{0}\right) \\
V_{s, 0}=V_{s}\left(s_{0}, h_{0}\right)
\end{gathered}
$$

The iterations through Equations (1-28)-(2-33) are continued until convergence on the flow rate is achieved. Then the critical flow limits are applied to the flow rate calculated by Equations (1-18) and (1-26); i.e., the flow rate is not allowed to exceed the critical flow rate.

Note that the critical flow rate does not depend on the conditions downstream of the valve as it should be, since, at critical flow, the downstream conditions are not communicated back to the inlet point. 
Although the critical flow algorithm is developed for the flow through a valve, it could also be applied to every flow in the Brayton cycle. Indeed, if a flow doesn't go through a valve, then $f_{\text {open }}=1$, and conditions at point 0 are the same as those at point 1 . Therefore, the iterative process will automatically converge after just one iteration. So, the critical flow is calculated and limitations are applied to every flow in the Brayton cycle.

Because of the possibility of reversed flow, the critical flow rate is calculated for both directions. The critical flow in the reversed direction, $\dot{m}_{c}^{-}$, is defined by the conditions which are outlet conditions for normal flow (Point 2 in Figure 1-4). The critical flow in the reversed direction is negative and the flow rate should always be between those two critical flows:

$$
\dot{m}_{c}^{-} \leq \dot{m} \leq \dot{m}_{c}
$$

According to Equations (1-32) and (1-33) the iterative scheme for the finding critical flow rate requires calculating density and sound speed as functions of enthalpy and entropy. Since the $\mathrm{CO}_{2}$ properties are defined as functions of temperature and density, using Equations (1-32) and (1-33) requires multiple iterations on the $\mathrm{CO}_{2}$ properties. Because of the complicated properties calculations, these iterations are too slow to be used in the dynamics code. Therefore, the following approach has been taken to eliminate iterations on $\mathrm{CO}_{2}$ properties. Tables of $\mathrm{CO}_{2}$ properties have been generated to provide pressure, temperature, density, and sound speed as a function of enthalpy and entropy. The tables cover the entire possible range of operating conditions for the cycle (temperatures from $230 \mathrm{~K}$ to $1100 \mathrm{~K}$, pressures from $0.05 \mathrm{MPa}$ to $50 \mathrm{MPa}$ ). The tables give the properties at fixed $\left(h_{i}, s_{j}, i, j=1 . .500\right)$ points; linear interpolation is used between the points. The tables cover supercritical, liquid, gas, and two-phase regions. In all regions, except the two-phase region, the properties are defined by the properties subroutines. In two-phase region, Henry's formula [4] is used to calculate the sound speed:

$$
\begin{aligned}
& V_{s}=\sqrt{\frac{\left[(1-x) \rho^{\prime \prime}+x \rho^{\prime}\right]^{2}+x(1-x)\left(\rho^{\prime}-\rho^{\prime \prime}\right)^{2}}{\frac{x\left(\rho^{\prime}\right)^{2}}{\left(V_{s}^{\prime \prime}\right)^{2}}+\frac{(1-x)\left(\rho^{\prime \prime}\right)^{2}}{\left(V_{s}^{\prime}\right)^{2}}}} \\
& h=x h^{\prime \prime}+(1-x) h^{\prime} \\
& s=x s^{\prime \prime}+(1-x) s^{\prime} \\
& \frac{1}{\rho}=\frac{x}{\rho^{\prime \prime}}+\frac{1-x}{\rho^{\prime}} \Rightarrow x=\frac{\frac{1}{\rho}-\frac{1}{\rho^{\prime}}}{\frac{1}{\rho^{\prime \prime}}-\frac{1}{\rho^{\prime}}}
\end{aligned}
$$


where $y^{\prime}$ and $y^{\prime \prime}$ denote liquid and gas properties, respectively.

The tables are based on the properties only and need to be generated only once. They are stored in a file and are read before the calculations start. The tables have also proved to be useful as a first guess for iterations in turbomachinery calculations which frequently employ calculations of the properties based on the specific enthalpy and entropy.

The tables are only used in the critical flow calculations. Other properties calculations, such as for Equations (1-15)-(1-18), are based on the exact properties subroutines and do not use the tables. Although using the tables instead of the exact calculations will result in some error, that error is not expected to be significant for the system performance for the following reasons. Critical flow is expected to occur in valves. The valves themselves are regulated by the Brayton cycle control. The control mechanisms monitor the system parameters and adjust the valve openings accordingly. However, valve opening itself does not affect the system performance - it is flow rate through the valve that is important. So, the control mechanisms effectively adjust the flow rate through the valves. Therefore, if there is an error in the critical flow rate calculations, the flow rate will be slightly different from the actual value, but the control system will still be adjusting the flow rate to a required value so that the resulting valve opening calculated by the control system may differ slightly from the actual value. Thus, the reported valve opening may not be exact due to utilization of the properties tables, but the flow rate still will be accurate such that the system performance is not affected.

Even with the properties tables, calculation of critical flow requires iterations on Equations (1-28)-(1-33). So, the critical flow rate is calculated only at the beginning of the time step. However, it is found that smooth variation of a valve open area is beneficial to the stability of the control system action. Therefore, the critical flow rate should also change smoothly during the time step. It is assumed that during the time step, the parameters of the flow in the opening are constant and the critical flow rate is proportional to the valve open area fraction according to Equation (1-28). The flow parameters and the critical flow rate are recalculated at the beginning of the next step.

Implementing Equations (1-25)-(1-26) will result in a numerical singularity when a valve approaches fully closed position $\left(f_{\text {open }} \rightarrow 0 \Rightarrow K_{v}^{\prime} \rightarrow \infty\right)$. To avoid this problem a threshold value for a valve opening is introduced. If the opening is below this threshold value then the flow is assumed to be critical, and calculations of Equations (1-25) and (126) are skipped. A 1\% open area is assumed for this threshold in the code.

\subsubsection{Turbomachinery Models}

The turbomachinery performance models are used to generate the turbomachinery maps which are used in the dynamics code. A detailed description of the compressor and turbine performance models is presented here. The performance models were developed for axial-flow turbine and compressor. Development of the centrifugal compressors models is described in Section 2.

Turbine and compressor off-design prediction models were developed using twodimensional (rather than complicated three-dimensional) models based on empirical 
cascade tests parameters [5] for simplicity and calculation speed. The main ideas and equations of these models are described below.

\subsubsection{Compressor Off-Design Performance Model}

An off-design performance prediction model was developed and used to generate the compressor performance maps for the dynamics code.

For the off-design model the following parameters are given:

- Inlet conditions

- Flow rate

- Stage geometry

- Rotational speed

- Blade profile and angles.

The goal of the performance model is to calculate the flow conditions inside the compressor and at the compressor outlet. The flow velocities and angles at each row are also calculated by the model.

The calculations start from the inlet. Given the flow conditions and flow rate, together with the design parameters of the IGV (flow area and IGV angle), that information specifies the flow velocity and direction at the IGV outlet or first stage inlet. Then, the relative to the blade flow velocity and angles are calculated at the stage inlet. The blade angle and flow angle define the incidence angle, $i$.

To determine the flow conditions at the outlet, iterations are required. It is assumed that the row-outlet density is known. The axial speed component at this point can then be found from the continuity equation with specified flow area by the design calculations.

The flow angle and the loss coefficient are to be found using the recommendations in [5]. The deviation angle is:

$$
\delta=\delta^{*}+\left[\frac{\partial \delta}{\partial i}\right]^{*}\left(i-i^{*}\right)+10\left(1-\frac{W_{z 2}}{W_{z 1}}\right)
$$

where

$$
\left[\frac{\partial \delta}{\partial i}\right]^{*}=\frac{1+\left(\sigma+0.25 \sigma^{4}\right)\left(\beta_{1} / 53\right)^{2.5}}{\exp (3.1 \sigma)} \text { is defined at design point. }
$$

The outlet flow angle is defined by as a sum of blade angle and deviation angle. The flow angle and axial speed component give the relative flow speed. The absolute speed, its components, and angles can be found.

The loss coefficient is expressed as a function of the design loss coefficient and incidence angle: 


$$
\bar{\omega}=\bar{\omega}_{m} \begin{cases}1+\xi^{2}, & -2 \leq \xi \leq 1 \\ 5-4(\xi+2), & \xi<-2 \\ 2+2(\xi-1), & \xi>1\end{cases}
$$

where

$$
\begin{aligned}
& \xi= \begin{cases}\left(i-i_{m}\right) /\left(i_{m}-i_{c}\right), & i<i_{m} \\
\left(i-i_{m}\right) /\left(i_{s}-i_{m}\right), & i \geq i_{m}\end{cases} \\
& \bar{\omega}_{m}=\text { loss coefficient at design point. }
\end{aligned}
$$

The flow conditions at the rotor outlet are defined by energy conservation equations, definition of loss coefficient, and equation of state (Equations (1-41)-(1-46)) with current values for the loss coefficient and flow speeds. The iterations on the rotoroutlet density are repeated, if necessary.

$$
\begin{aligned}
& h_{02}^{\prime}=h_{01}^{\prime}=h_{1}+\frac{W_{1}^{2}}{2} \\
& p_{01}^{\prime}=p\left(h=h_{01}^{\prime}, s=s_{1}\right) \\
& p_{02}^{\prime}=p_{01}^{\prime}-\bar{\omega}\left(p_{01}^{\prime}-p_{1}\right)-\Delta p_{0 c}
\end{aligned}
$$

where

$\bar{\omega}=$ loss coefficient,

$\Delta p_{0 c}=$ tip clearance gap total pressure loss.

$$
\begin{gathered}
\Delta p_{0 c}=\Delta p \frac{\dot{m}_{c}}{\dot{m}} \\
\dot{m}_{c}=\bar{\rho} U_{c} Z \delta_{c} c \cos \gamma, \\
U_{c}=\frac{0.816}{N_{\text {row }}^{0.2}} \sqrt{\frac{2 \Delta p}{\bar{\rho}}} \\
s_{2}=s_{02}^{\prime}=s\left(p=p_{02}^{\prime}, h=h_{02}^{\prime}\right) \\
h_{2}=h_{02}^{\prime}-\frac{W_{2}^{2}}{2} \\
p_{2}=p\left(h=h_{2}, s=s_{2}\right), \quad T_{2}=T\left(h=h_{2}, s=s_{2}\right)
\end{gathered}
$$

The same procedure is repeated for the stator and then for every stage until the outlet conditions are found.

Based on the inlet conditions and the outlet pressure, the outlet enthalpy in an isentropic process is calculated: 


$$
h_{s, \text { out }}=h\left(p=p_{\text {out }}, s=s_{\text {in }}\right)
$$

The total-to-static compressor efficiency is:

$$
\varepsilon=\frac{h_{s, \text { out }}-h_{\text {in }}-\frac{C_{\text {in }}^{2}}{2}}{h_{\text {out }}+\frac{C_{\text {out }}^{2}}{2}-h_{\text {in }}-\frac{C_{\text {in }}^{2}}{2}}
$$

To calculate the stall conditions, the stall criterion recommended by [5] is used:

$$
W_{R E}<W_{R E}^{\min }
$$

where

$$
\begin{aligned}
& W_{R E}=\sqrt{\frac{p_{02}^{\prime}-p_{2}}{p_{01}^{\prime}-p_{1}}}=\text { equivalent relative velocity ratio across the blade, } \\
& W_{R E}^{\min }=k \frac{\left(0.15+11 t_{b} / c\right) /\left(0.25+10 t_{b} / c\right)}{1+0.4[\theta \sigma /(2 \sin (\theta / 2) \cos \gamma)]^{0.65}}=\text { stall velocity ratio, }
\end{aligned}
$$

with condition $\theta \sigma /(2 \sin (\theta / 2) \cos \gamma) \geq 1.1$ applied,

$$
k=\left\{\begin{array}{l}
1, \quad D_{e q} \leq 2.2 \\
\left(2.2 / D_{e q}\right)^{0.6}, \quad D_{e q}>2.2
\end{array}\right. \text {. }
$$

In order to have a quantitative equivalent of Criterion (1-49), the stall coefficient is introduced:

$$
f_{\text {stall }}=\frac{W_{R E}}{W_{R E}^{\min }}
$$

The stall criterion would then be $f_{\text {stall }}<1$, and $f_{\text {stall }}$ will show how far the compressor is from the stall conditions.

Choke conditions occur when the calculated Mach number (i.e., ratio of the local speed to the speed of sound) at any row inlet or outlet reaches unity. Therefore, the maximum Mach number in the compressor is used to measure how far the compressor is from the choking condition.

To demonstrate the application of the performance model, the example of the performance maps with fixed inlet conditions are calculated for each compressor. Figure 1-5 and Figure 1-6 show such performance maps for Compressors No. 1 and 2, respectively. 


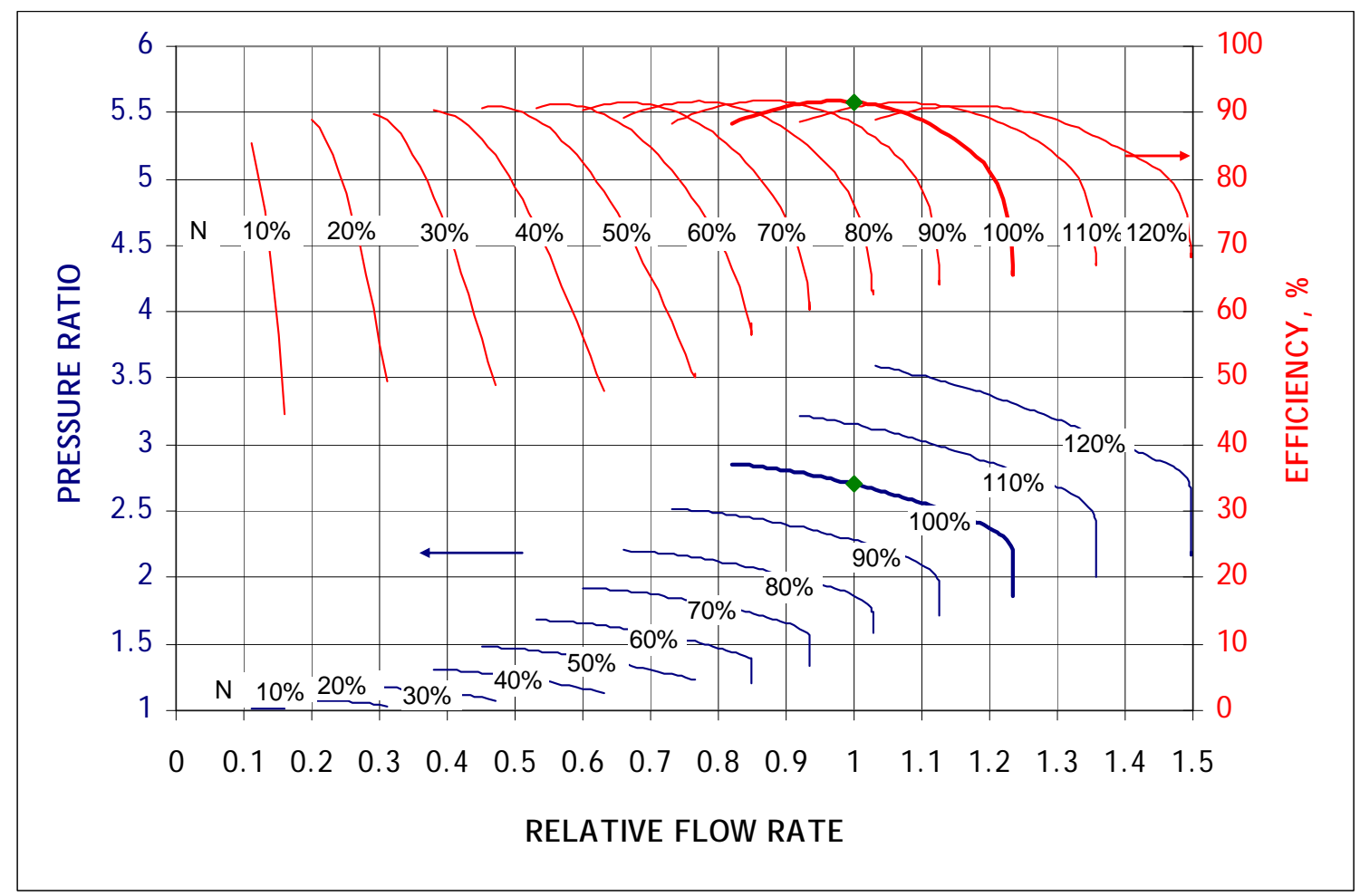

Figure 1-5. Example of Compressor No. 1 Performance Map.

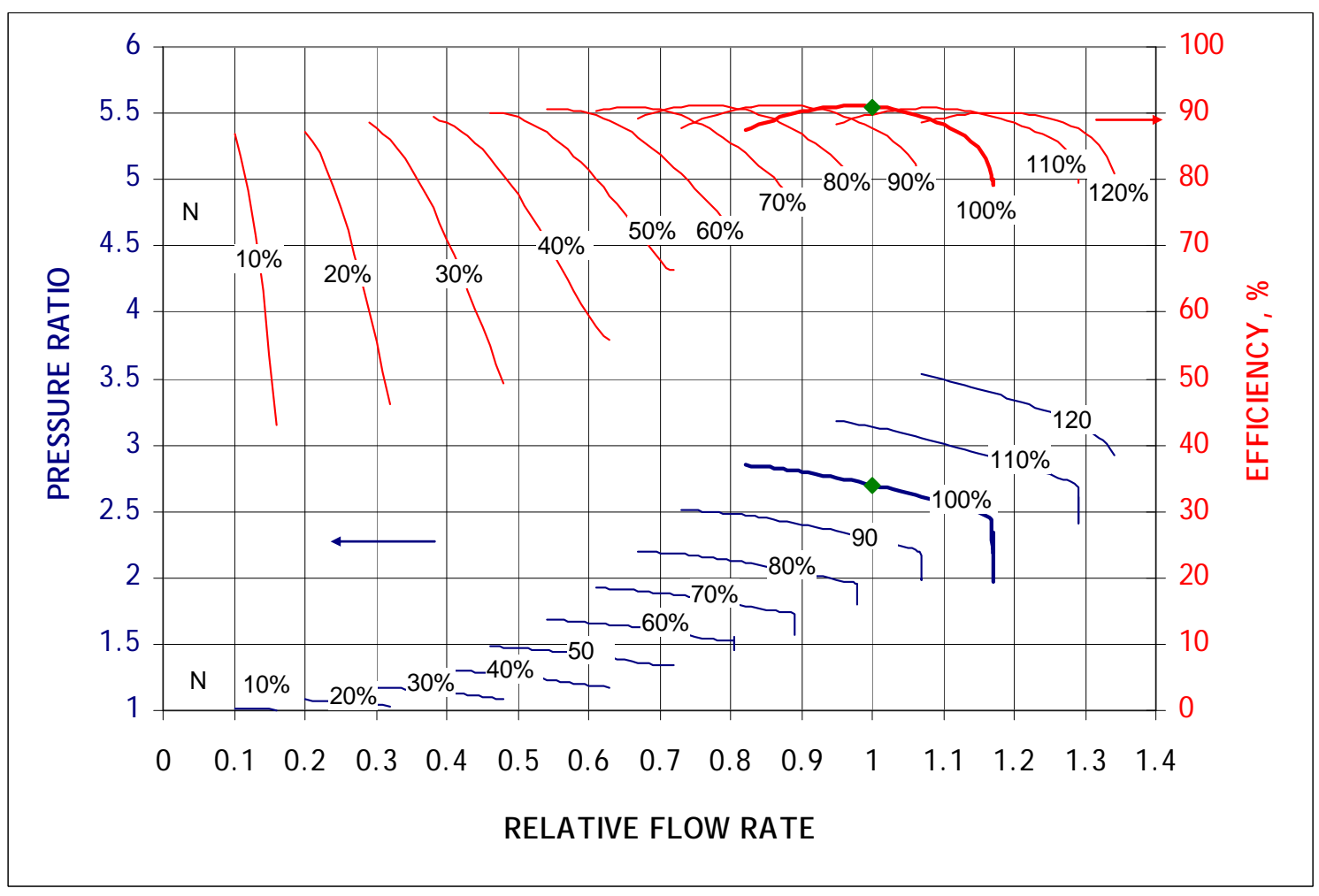

Figure 1-6. Example of Compressor No. 2 Performance Map. 


\subsubsection{Turbine Models}

The turbine performance is very similar to the compressor model described above. The main differences include the row order (nozzles are followed by rotors in a turbine), absence of the inlet guiding vane, and the blade loss model.

The loss model for the turbine blades is implemented following the recommendations of [6]. This model uses the loss correlations presented by [7]. The advantage of the model is that it includes effects such as:

- Blade profile losses,

- Mach number correction,

- Shock losses,

- Tip clearance leakage,

- Reynolds number correction,

- Secondary flow losses, and

- Trailing edge losses.

The total loss coefficient is calculated as a sum of loss coefficients from profile, secondary, and tip leakage losses:

$$
Y=Y_{p}+Y_{s}+Y_{t i p}
$$

The loss coefficient has the same definition as a compressor loss coefficient, $\bar{\omega}$, in Equation (1-43), except that the tip leakage loss is included into the loss coefficient and does not appear in Equation (1-43) written for the turbine blades.

The components of loss coefficients are calculated based on References [7] and [8].

The turbine map generated for fixed inlet conditions is presented in Figure 1-7. 


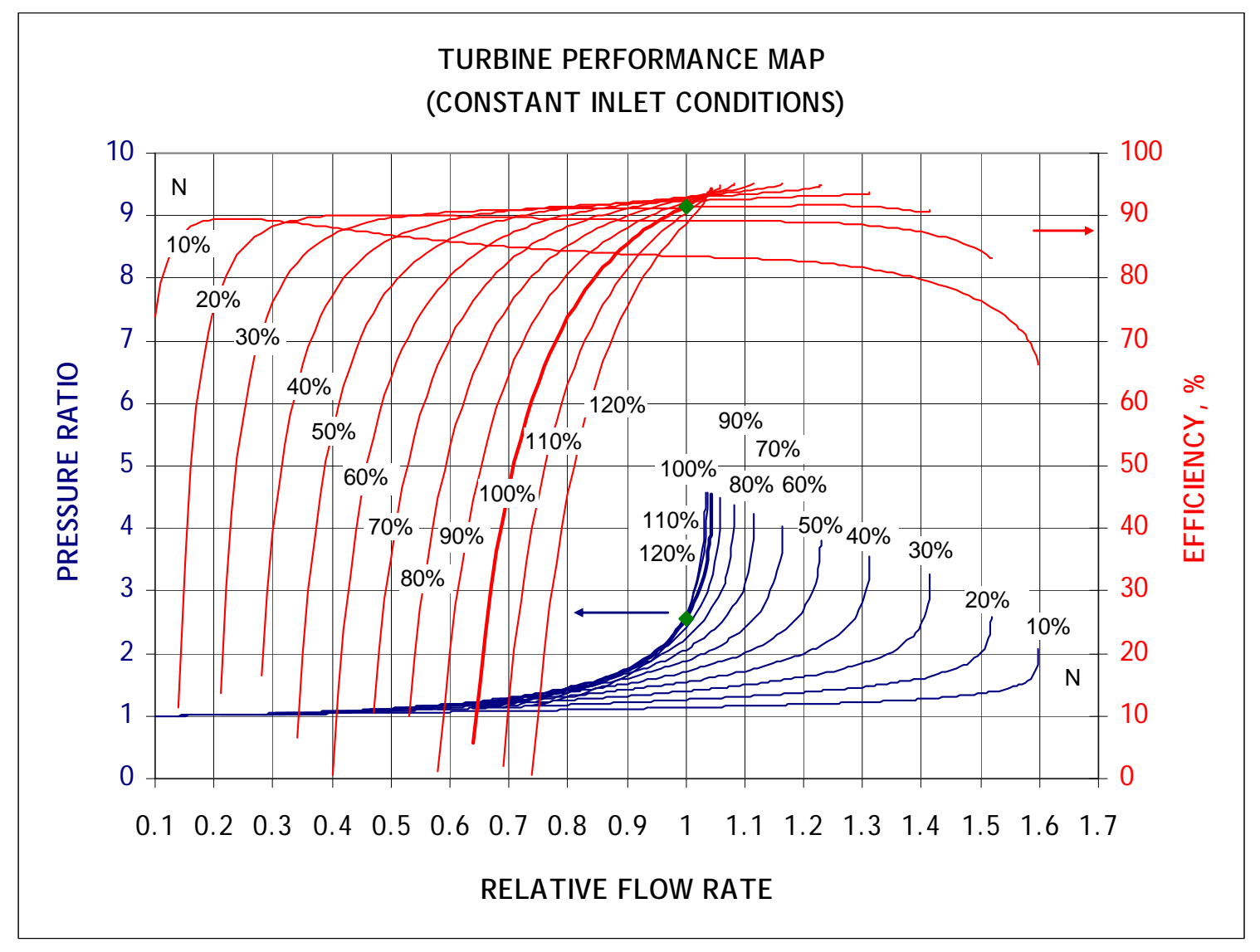

Figure 1-7. Example of Turbine Performance Map.

\subsubsection{Turbomachinery in the Plant Dynamics Model}

The common approach in turbomachinery modeling is an instantaneous response assumption [2]. Under this assumption, the flow rate and outlet parameters react instantaneously to changes in the inlet and outlet pressure and/or rotational speed. The steady-state off-design performance model is used to calculate the turbomachinery behavior under this approach. However, those off-design models usually involve multiple layers of iterations on fluid properties, speeds, and losses and, therefore, are much too slow to be directly used in dynamic calculations. The iterations could be very slow for $\mathrm{CO}_{2}$ due to rapid properties variations.

Instead, performance maps are generated for each turbomachinery component before the dynamic calculations are performed. These maps are then used in the dynamics model. The maps should cover the whole possible range of parameter variations to avoid extrapolation. The parameters which define the turbomachinery state are:

- Inlet temperature;

- Inlet pressure;

- Outlet pressure; and

- Rotational speed. 
Based on these parameters, the steady-state off-design performance model calculates all other parameters and conditions. Of the calculated parameters, the mass flow rate though the turbomachinery and outlet temperature (or specific enthalpy) are used in the dynamics model. So, the goal of the performance maps is to generate a behavior law for flow rate and outlet temperature for variations in each of the four parameters listed above. The traditional approach for ideal gas cycles has been to reduce the number of varying parameters to two (usually, pressure ratio and temperaturecorrected rotational speed) using the fact that the fluid follows the ideal gas law. Therefore, the turbine and compressor maps are usually generated as functions of two variables in traditional approaches.

It is known, however, that carbon dioxide does not follow the ideal gas law, especially near the critical point where Compressor No. 1 is operated. For example, a very small change in inlet pressure, say $7.5 \mathrm{MPa}$ vs. $7.4 \mathrm{MPa}$ at design, causes a very significant change in inlet density $\left(594 \mathrm{~kg} / \mathrm{m}^{3}\right.$ vs. $369 \mathrm{~kg} / \mathrm{m}^{3}$, for this example and design temperature). This change in density will disturb the fluid velocities resulting in significant differences in outlet pressure (30.6 MPa vs. 20.0 MPa) for the same flow rate through the compressor and the same rotational speed. Therefore, the flow rate could not be defined as a function of rotational speed and pressure ratio only (the pressure ratios are 4.08 and 2.70, in this example). The inlet temperature has a similar effect for $\mathrm{CO}_{2}$. Although it could theoretically be possible to find the non-dimensional parameters which will allow a reduction of the number of variables even for $\mathrm{CO}_{2}$, finding these parameters could be very difficult, if at all successful. Instead, an approach is adopted that involves generation of performance maps for individual variations of each of the four parameters involving four-dimensional arrays. Fortunately, modern computers can generate, store, and work with million-element arrays (e.g., 40 points for each parameter requires $40^{4}=2.56 \cdot 10^{6}$ elements).

Four-dimensional performance maps are generated for the turbine and two compressors. These maps provide the flow rate, outlet temperature, maximum Mach number (for checking choke conditions), and stall parameter (for checking compressor stall conditions) for each value of rotational speed, inlet temperature, and inlet and outlet pressures. The parameters between the points are obtained in the dynamics model by linear interpolation between the map points.

Figure 1-8 shows the example of the performance map for Compressor No. 1. It shows the flow rate (relative to nominal) for each point of inlet and outlet pressure with the design values for inlet temperature and rotational speed. The points in inlet pressure represent values of inlet pressure with the design value in the middle. The points for outlet pressure are obtained by dividing the range between stall and choke conditions into a fixed number of intervals such that the actual values for outlet pressure at each point are different for different inlet conditions (these values are stored in a separate array). Similar maps are constructed for each point in inlet temperature and rotational speed to compose the complete performance map for the flow rate through Compressor No. 1. Similarly, the maps for outlet temperature, maximum Mach number, and stall criteria (for compressors) are obtained for the turbine and each compressor.

While a significant amount of computational time is required to generate the fourdimensional maps, the maps only need to be generated once. The utilization of the 
relative to steady-state, rather than absolute, parameters allows use of the same maps even if design conditions are different ${ }^{1}$. The usage of the four-dimensional arrays has proven to be fast enough for the dynamics code.

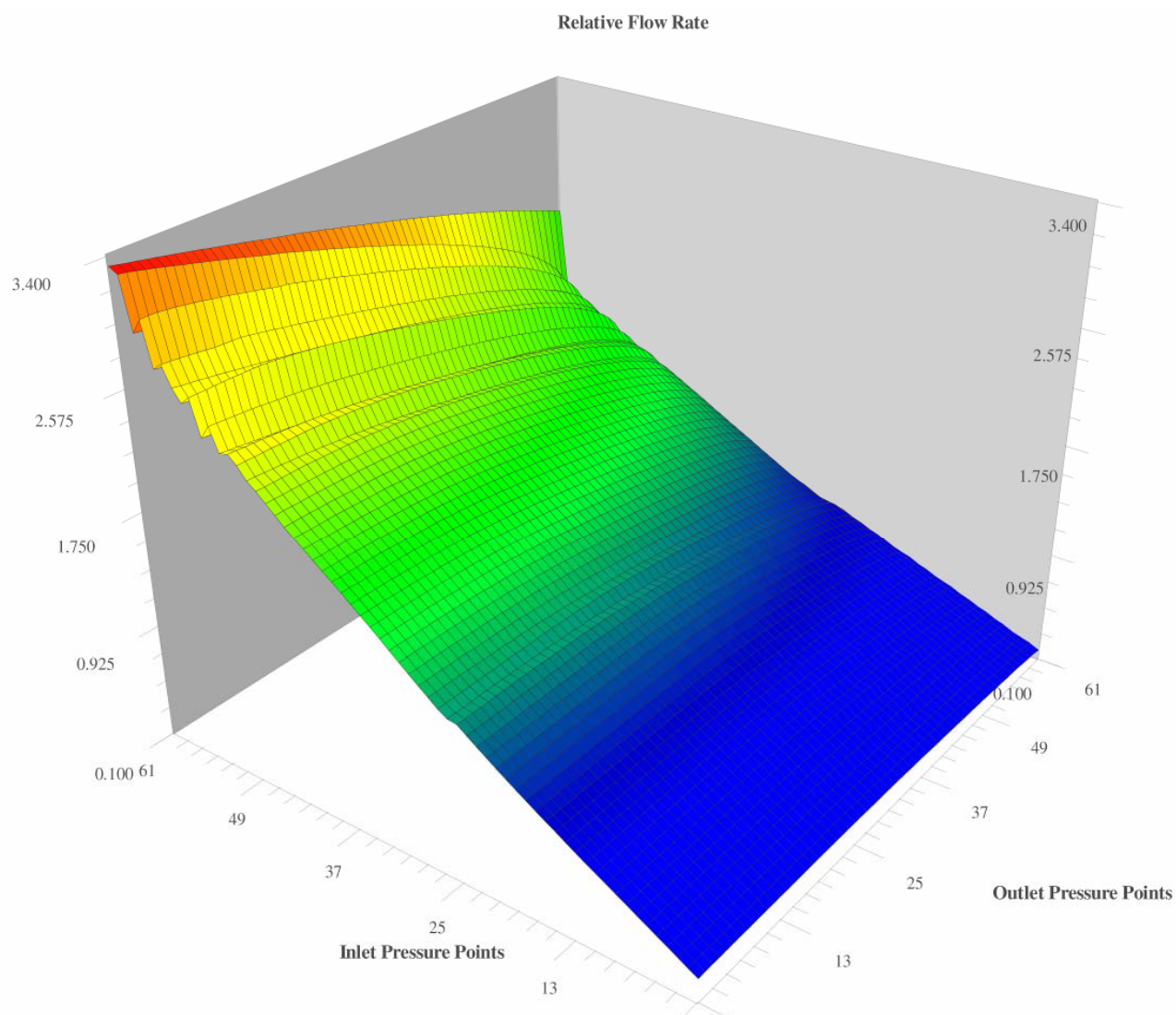

Figure 1-8. Example of Compressor No. 1 Performance Map.

\subsubsection{Turbomachinery Shaft Dynamics}

The turbomachinery rotor dynamics equation defines the change in the turbomachinery rotational speed [2]:

$$
\frac{\partial \omega}{\partial t}=\frac{W_{g e n}-W_{g r i d}}{I \omega}
$$

where

$$
\omega=2 \pi n_{r}=\text { rotational speed, }
$$

\footnotetext{
${ }^{1}$ The turbomachinery design is integrated into the steady-state code which is based on several layers of iterations with finite convergence criteria. Therefore, the steady-state results, including the turbomachinery design conditions, are slightly different at each start of the dynamics calculations.
} 
$W_{\text {gen }}=$ generator power (see below),

$W_{\text {grid }}=$ demand from the grid,

$I=$ total moment of inertia.

The generator power is defined as the difference between the power produced by the turbine $\left(W_{T}\right)$ and power consumed by the two compressors $\left(W_{C 1}, W_{C 2}\right)$ with accounting for the mechanical losses $\left(W_{l}\right)$, generator efficiency $\left(\varepsilon_{g e n}\right)$, and power input for water pump for the cooling water provided to the cooler:

$$
W_{\text {gen }}=\left(W_{T}-W_{C 1}-W_{C 2}-W_{l}\right) \varepsilon_{\text {gen }}-W_{\text {water }}
$$

The component power is calculated as the flow rate through the component times the change in specific enthalpy across the component. The default mechanical losses are assumed to be $1 \%$ of the full generator power and proportional to the square of the rotational speed. The default generator efficiency is assumed to be $98.5 \%$. The grid demand is given as a function of time. The total moment of inertia is a sum of the moments for the turbine, compressors, and generator rotors. The moment of inertia is defined as [9]:

$$
I=\int r^{2} d m
$$

For a solid cylinder of radius $r$, length $L$, mass $m$, and density $\rho$, it is equal to:

$$
I_{c y l}=\frac{1}{2} m r^{2}=\frac{1}{2} \pi \rho L r^{4}
$$

Equation (1-55) is used to obtain the moment of inertia for each stage of the turbine and compressor. For example, the moment of inertia of a compressor stage is a sum of moments for each rotating part ${ }^{1}$ as shown in Figure 1-9.

$$
\begin{aligned}
& I_{\text {comp }}=\sum_{i=1}^{N_{\text {st }}}\left(I_{\text {shaft }_{i}}^{\text {rot }}+I_{\text {hub }_{i}}^{\text {rot }}+I_{\text {blades }_{i}}^{\text {rot }}+I_{\text {shaff }_{i}}^{\text {stat }}\right) \\
& I_{\text {shaff }_{i}}^{\text {rot }}=\frac{1}{2} \pi \rho_{\text {shaft }} \text { Chord }_{i}^{\text {rot }} r_{\text {shaft }}^{4} \\
& I_{\text {shaff }_{i}}^{\text {stat }}=\frac{1}{2} \pi \rho_{\text {shaft }} \text { Chord }_{i}^{\text {stat }} r_{\text {shaft }}^{4}
\end{aligned}
$$

\footnotetext{
${ }^{1}$ At this stage of the turbomachinery design, it is not clear whether to include the stator row hub into rotating parts or not. In any case, as it will be shown below, the moments of inertia of turbine and compressors are small compared to generator's moment of inertia. So the effect of the stator hub is not significant for the total moment of inertia.
} 


$$
\begin{aligned}
& I_{\text {hub }_{i}}^{\text {rot }}=\frac{1}{2} \pi \rho_{\text {hub }} \operatorname{Chord~}_{i}^{\text {rot }}\left(r_{\text {hub }}^{4}-r_{\text {shaft }}^{4}\right) \\
& I_{\text {blades }_{i}}^{\text {rot }}=\frac{1}{2} \pi \rho_{\text {blade }} K_{\text {blade }} \operatorname{Chord~}_{i}^{\text {rot }}\left(r_{\text {tip }}^{4}-r_{\text {hub }}^{4}\right)
\end{aligned}
$$

where

$\rho=$ material density,

$r=$ radius,

$K_{\text {blade }}=$ blade row "porosity" (see below).

The blade row "porosity" is a ratio of the volume occupied by the blades to the total volume between the hub and tip. The blade's total volume is calculated under the assumption that the blades are arcs with an average thickness equal to half of the maximum thickness.

$$
K_{\text {blade }}=\sigma \frac{t_{b}}{2 c} \frac{\theta}{2 \sin \frac{\theta}{2}}
$$

where (see figure to the right)

$$
\begin{aligned}
& \sigma=\frac{c}{s}=\text { blade solidity } \\
& c=\text { blade chord } \\
& s=\text { blade spacing, } \\
& t_{b}=\text { maximum blade thickness }, \\
& \theta=\text { blade angle. }
\end{aligned}
$$
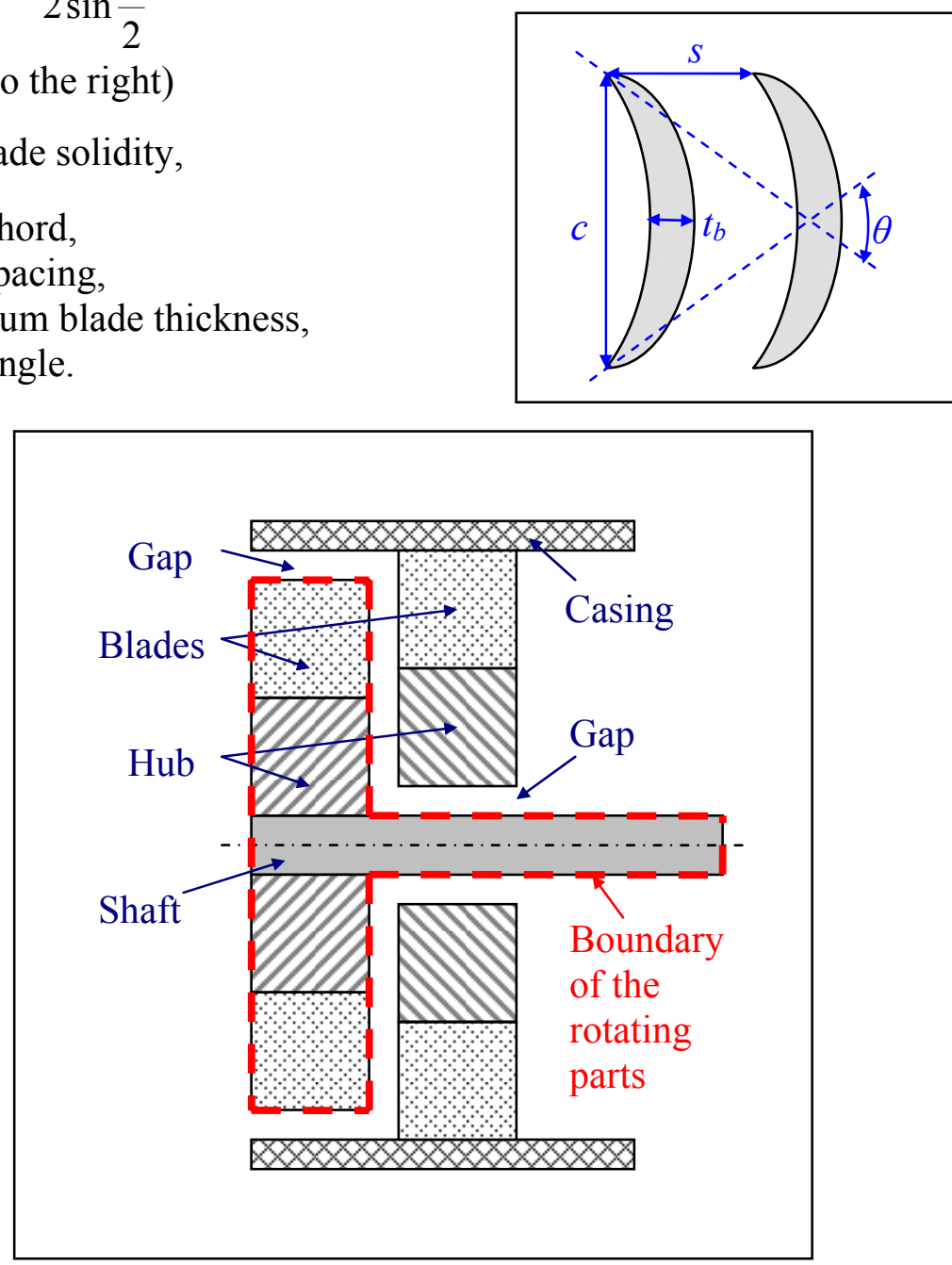

Figure 1-9. Compressor Stage for Moment of Inertia Calculations. 
The moment of inertia for the generator rotor is calculated using Equation (1-55) by interpolating data for existing generators. Table 1-1 shows the calculated moments of inertia for the turbine, compressors and generator.

\section{Table 1-1. Moments of Inertia}

$\left(\mathrm{kg}-\mathrm{m}^{2}\right)$

\begin{tabular}{|c|c|c|c|c|}
\hline Generator & Turbine & Compressor \#1 & Compressor \#2 & TOTAL \\
\hline 4706 & 223.451 & 4.931 & 14.456 & $\mathbf{4 9 4 8 . 8}$ \\
\hline
\end{tabular}




\subsection{Brayton Cycle Automatic Control}

The Brayton cycle automatic control system adjusts the cycle in such a way that the plant produces electricity in an amount exactly equal to the grid demand. The Brayton cycle control system for STAR-LM incorporates several control mechanisms as shown in Figure 1-10.

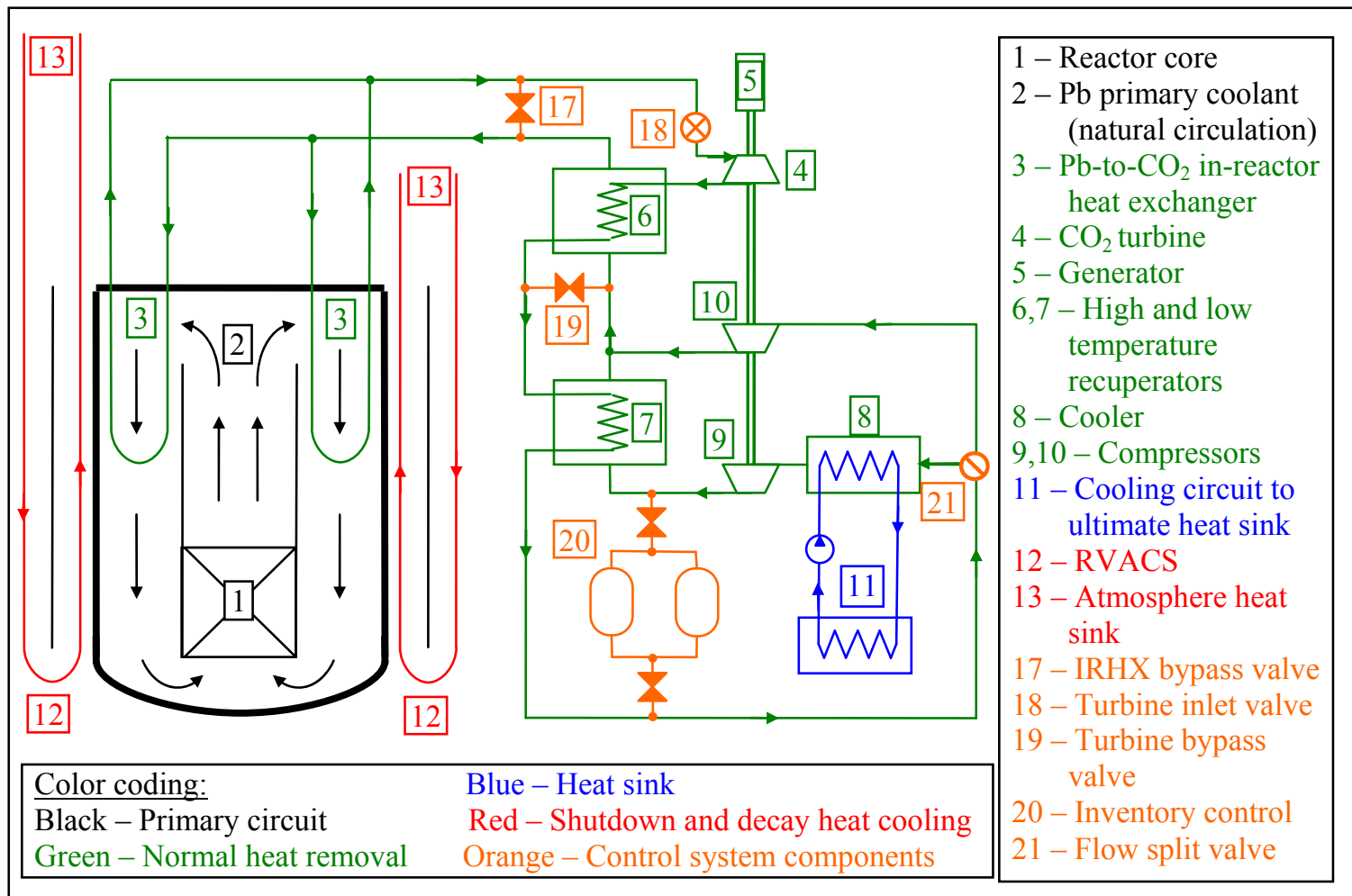

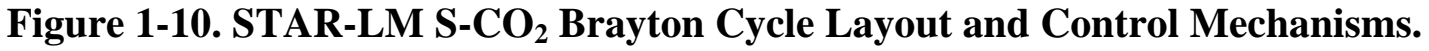

One of the motivations for STAR-LM development was to design a reactor which could supply electricity to customers who are not connected to a major electrical grid. Therefore, it is assumed in the analysis that the STAR-LM turbogenerator rotational speed dictates the grid frequency such that the generator works in an asynchronous mode at all power levels. Thus, the rotational speed of the generator (and the turbine and compressors, since they are all on one shaft) has to be actively controlled all of the time. The turbogenerator rotational speed can be controlled by the power supplied by the turbine to the generator. If the generator power exceeds the demand from the grid, then the excess of energy is stored as the kinetic energy of the rotating components and the shaft starts to rotate faster. Therefore, the rotational speed can be controlled by adjusting the net power produced by the turbine (turbine work minus the work demand of the compressors). 


\subsubsection{Turbine Bypass Control}

The common practice for gas Brayton cycles is to use turbine bypass for turbomachinery speed control. The same approach is used for this cycle. If the generator power exceeds the grid demand, the turbine bypass valve opens reducing the flow rate through the turbine and, therefore, decreasing the turbine work. The turbine bypass automatic control monitors the rotational speed of the turbogenerator. It calculates the difference between the actual rotational speed and its specified value. It is assumed that it also calculates the derivative and integral value of this difference. The bypass valve action, valve opening or closing, is calculated based on the three parameters representing proportional, differential, and integral control as defined by Equation (1-61).

$$
f_{T B P}^{\prime}=k_{p}^{T B P} E_{T B P}+k_{d}^{T B P} E_{T B P}^{\prime}+k_{i}^{T B P} \int_{0}^{t} E_{T B P}(t) d t
$$

where

$f_{T B P}^{\prime}=$ rate of change of open area fraction, $f_{\text {open }}^{T B P}$, or speed at which the valve is opening or closing,

$E_{T B P}=\frac{n_{r}(t)-n_{r}^{\text {spec }}}{n_{r}^{\text {spec }}}=$ deviation of the rotational speed, $n_{r}$, from its specified value,

$k_{d}, k_{p}, k_{i}=$ user-defined coefficients of control.

The coefficients for the proportional, differential, and integral parts of the control are defined by the user in the input file. They are selected to achieve a satisfactory response from the control system.

The derivative of deviation is generally calculated as the difference between the current value and the value at the previous time step divided by the time step.

$$
E^{\prime}=\frac{E(t)-E(t-\Delta t)}{\Delta t}
$$

However, the derivative for the turbine bypass deviation is known at every time step since the derivative of the shaft rotational speed is calculated by the shaft dynamics Equation (1-52).

$$
E_{T B P}^{\prime}=n_{r}^{\prime}
$$

The deviation integral is calculated as a sum of error values multiplied by the time step for all time steps from the beginning of a transient.

$$
\int_{0}^{t} E_{T B P}(t) d t=\sum_{i} E_{T B P}(t) \Delta t_{i}
$$


The valve open fraction at the end of the time step is calculated based on the value at the beginning of the time step and the opening or closing rate.

$$
f_{\text {open }}^{T B P}=\left(f_{\text {open }}^{T B P}\right)_{t=0}+f_{T B P}^{\prime} \Delta t
$$

The limitations on the open area fraction (between 0 and 1) and valve opening and closing rates (defined by the user) are applied to the results obtained from Equation (161).

It should be noticed here that opening the bypass valve reduces the amount of useful work performed by the turbine. Therefore, the valve should be closed during operation at full power. However, it is found to be beneficial from the stability point of view to maintain a small bypass flow (valve open fraction is much less than $1 \%$ ). This small bypass flow does not show any significant effect on the generator output while allowing usage of turbine bypass control for very small changes (uncertainties) in the generator output. Although inventory control could be used for small power increase, that control mechanism is shown not to be fast enough for turbogenerator speed control.

If the calculated value of the turbine bypass valve open area fraction is less than zero then the valve is closed $(f=0)$ and the remaining value (i.e., $-f)$ is added to the inventory control action (Equation (1-67) below) to simulate shaft speed control by inventory addition. This would be the case, for example, when the plant operates above $100 \%$ load as described below.

\subsubsection{Inventory Control}

Quasi-static calculations [10] showed that inventory control is the preferable control mechanism, since it provides the highest cycle efficiency at reduced loads. At the same time, during the development of the dynamic control system, it was realized that inventory control could not be fast enough to regulate the turbomachinery speed. Rapid removal of the $\mathrm{CO}_{2}$ inventory from the cycle introduces large disturbances in the compressor conditions causing stalling or choking in one of the compressors. Thus, turbine bypass control is utilized for small changes in loads as a means of shaft speed control. In order to eliminate dual action from both control mechanisms, the control system logic is specified such that turbine bypass controls the cycle from $100 \%$ to $90 \%$ load and inventory control regulates the cycle below 90\%. Turbine bypass control still regulates the shaft speed at any power level. In order to implement an automatic control on the $\mathrm{CO}_{2}$ inventory in the cycle, an inventory control table is generated. The inventory control table specifies how much $\mathrm{CO}_{2}$ mass needs to be removed from the cycle for each power level. The table is generated based on quasi-static calculations. Any inaccuracy in the table will result in a mismatch between the generator output and grid demand. This imbalance will be reflected in the shaft rotational speed, and, therefore, will be regulated by the turbine bypass automatic control.

The automatic inventory control system monitors the $\mathrm{CO}_{2}$ mass in the inventory tanks and opens and closes inlet and outlet valves by an approach similar to Equation (162 ), in order to match the required mass obtained from the control table. Since opening an inlet or outlet valve adds or removes $\mathrm{CO}_{2}$ inventory from the tank (acting as derivative 
of the inventory), the inventory control system calculates valve openings (not their derivatives).

$$
f_{I N V}=k_{p}^{I N V} E_{I N V}+k_{d}^{I N V} E_{I N V}^{\prime}+k_{i}^{I N V} \int_{0}^{t} E_{I N V}(t) d t+\left|f_{\text {open }}^{T B P}\right|
$$

where

$$
\begin{aligned}
& f_{I N V}=\text { total action from inlet and outlet valves (see below), } \\
& \begin{aligned}
E_{I N V}= & \frac{\Delta M_{t}^{\text {spec }}-\Delta M_{t}(t)}{M_{t, t=0}}=\text { deviation of the mass addition to the inventory tank, } \\
\left|f_{\text {open }}^{T B P}\right|= & \text { remaining action from turbine bypass control (used here only if } \\
& \quad \text { calculated } f_{\text {open }}^{T B P} \text { is less than zero). }
\end{aligned}
\end{aligned}
$$

The deviation derivative and integral are calculated as shown in Equations (1-63) and (1-65).

If the inventory control action calculated by Equation (1-67) is positive $\left(\Delta M_{t}\right.$ is less than a required value) then the inlet valve opens; otherwise the outlet valve opens:

$$
\begin{cases}f_{\text {open }}^{I N V i}=f_{I N V}, & \text { if } f_{I N V}>0 \\ f_{\text {open }}^{I N V o}=-f_{I N V}, & \text { if } f_{I N V}<0\end{cases}
$$

It is shown [10] that the inventory control system has limited range mostly due to inventory tank volume limitations - inventory control is to be used from $90 \%$ to $50 \%$ load. The control table is generated to cover this range; if the load falls below $50 \%$, the control table will still provide the $50 \%$ load value such that inventory control will not take any action. Instead, other control mechanisms will be initiated as described below.

It is also shown that inventory control is capable of increasing the plant power above $100 \%$ nominal, if $\mathrm{CO}_{2}$ inventory is added to the cycle. However, the results demonstrated that this power increase results in increase of the core structure temperatures beyond the safety limits for steady-state operation. Still, the capability of short-term power increase from the cycle is demonstrated, mostly as an exercise. The inventory control table is extended up to $110 \%$ for these purposes.

\subsubsection{Turbine Throttle Valve Control}

For loads below 50\% of nominal full power, turbine throttle valve control is initiated. Again, based on quasi-static calculations, a control table is generated showing the required pressure drop across the throttle valve as a function of the grid demand. Similar to the inventory control table, any inaccuracy in the throttle valve control table will be compensated by turbine bypass control. The turbine throttle valve automatic control system calculates the difference between the actual pressure drop across the valve and the required value, and adjusts the valve opening. 


$$
f_{T I N}^{\prime}=k_{p}^{T I N} E_{T I N}+k_{d}^{T I N} E_{T I N}^{\prime}+k_{i}^{T I N} \int_{0}^{t} E_{T I N}(t) d t
$$

where

$f_{T I N}^{\prime}=$ rate of change of open area fraction, $f_{\text {open }}^{T I N}$, or speed at which the valve is opening or closing,

$E_{T I N}=\frac{\Delta p_{T I N}^{s p e c}-\Delta p_{T I N}(t)}{p_{T I N}}=$ deviation of the pressure drop across the turbine inlet valve, $\Delta p_{T I N}$, from its specified value.

The deviation derivative and integral are calculated as shown in Equations (1-63) and (1-65). The valve open area is calculated similar to Equation (1-66).

\subsubsection{Flow Split Control}

In parallel to the turbine throttle valve, a flow split control is activated. This control monitors the conditions in the compressors. If an approach to stall or choke is detected in any of the compressors, the flow split between the compressors is adjusted. To regulate the flow split, a throttle valve before Compressor No. 2 is utilized. The valve action is calculated as a sum of the actions from each limiting condition (stall or choke). For example, if the Compressor No. 1 stall parameter, $f_{\text {stall }}$, is below $1.1\left(f_{\text {stall }}=1\right.$ means stall), the control system will start closing the valve before Compressor No. 2 to allow for more flow through Compressor No. 1. The closing rate will be increasing as the stall parameter approaches unity.

$$
\left(f_{C 2 I N}^{\prime}\right)_{C 1 \text { stall }}=-\frac{1.1-f_{\text {stall }}^{C 1}}{1.1-1} f_{C 2 \text { IN }}^{c l . \text { lim. }}
$$

where

$\left(f_{C 2 I N}^{\prime}\right)_{C 1 \text { stall }}=$ part of the Compressor No. 2 inlet valve opening or closing rate due to stall conditions at Compressor No. 1,

$f_{C 2 I N}^{c l . \lim .}=$ maximum closing rate for the valve.

It is shown [10] that the compressor operating ranges are the main limiting parameters for every control mechanism (except inventory control) range. Since flow split control is used to prevent limiting conditions in the compressors, its utilization extends the range of the turbine bypass and turbine inlet valve controls.

The turbine throttle valve (with flow split control) controls the cycle between $50 \%$ and $20 \%$ load. For smaller loads, turbine bypass control will automatically regulate the generator output by controlling the shaft speed. 


\subsubsection{IRHX Bypass Control}

It is show that the control mechanisms described above are sufficient to control the plant over the full load range (between $0 \%$ and $100 \%$ loads). Therefore, an automatic control for IRHX bypass is not implemented in the code. It could be done similarly to turbine inlet valve control, if a need for automatic IRHX bypass control arises in the future. At this time, a "manual" control action is simulated, i.e., the user can specify the valve open area fraction as a function of time (similar tables for other controls are also specified in the input file).

\subsubsection{Plant Operation Limits}

The plant control system has to allow plant operation without exceeding the safety and operational limits. These limits include:

- Peak temperatures in the core, such as the coolant boiling temperature and cladding temperature limit,

- Coolant freezing,

- Compressor stall and choking conditions,

- Turbine choking,

- Excessive $\mathrm{CO}_{2}$ pressures and temperatures, and

- Excessive shaft rotational speeds.

\subsection{Simulation of Plant Control}

The normal operational transients are simulated to test the plant control system. The transient simulate the variation of the grid demand anticipated for normal plant operation. These variations include full power operation (no change), small instantaneous (step) changes, and smooth (linear) load changes.

The grid demand (load) is specified by user in the input file as a table of grid loads versus time at a finite number of points; a linear load change is assumed between those points. The load is equal to the first value for times before the first point and is equal to the last value for times after the last point.

Any transient calculations are preceded by initial system stabilization. During that time the same dynamics equations are solved; however, no transient initiator is introduced into the system and no control system action is initiated. The reactor power is equal to the steady state value and is not calculated by the reactor kinetics subroutines. The shaft rotational speed is also constant at the steady-state value and is not calculated by the shaft dynamics equation. It is found that 300-500 s are enough to achieve stabilized conditions. It is assumed that the transient (system disturbance) starts at $t=0 \mathrm{~s}$; the initial system stabilization is reported for negative times. For the positive times, all dynamics equations are solved, including calculation of the reactor power by the kinetics 
subroutines and shaft rotational speed by the shaft dynamics equation, and the control systems are functional.

\subsubsection{Result Graphs Explanation}

Next section presents the results obtained with the dynamics model. The model solves a large number of equations to find the time-dependent values of parameters which characterize the system. Therefore, a large number of parameters is reported in the output graphs in order to understand the system behavior. To avoid confusion, different parameters are reported on different graphs. A total of 20 graphs is selected as necessary and more or less sufficient to characterize the system behavior in a transient. The graphs are presented as four on a page. Below are the brief descriptions of the parameters for the graphs grouped by page (in left-to-right, then down order).

\section{Page 1:}

- Heat balance in reactor

o Reactor core power (Q_Rx)

o Heat removal rate by $\mathrm{CO}_{2}$ in $\operatorname{IRHX}\left(\mathrm{Q} \_\mathrm{RHX}\right)$

o Heat removal rate by air in RVACS (Q_air)

- Coolant speed in core

o Average coolant speed in core $\left(\mathrm{u}_{-} \mathrm{Pb}\right)$

- Coolant temperatures in core

o Average core-outlet temperature ( $T_{-}$out)

0 Average core-inlet temperature $\left(\mathrm{T}_{-}\right.$in $)$

- Coolant temperatures in $H X$

o IRHX-inlet temperature (Tin_HX)

o IRHX-outlet temperature (Tout_HX)

\section{Page 2:}

- Peak temperatures in core

o Peak fuel temperature (Tf_max)

o Peak cladding temperature (Tcl_max)

o Peak coolant temperature (TPb_max)

- Peak RV temperatures

o Maximum reactor vessel inner surface temperature (TRVi_mx)

o Maximum average reactor vessel temperature (TRV_mx)

o Maximum reactor vessel outer surface temperature (TRVo_mx)

- Average temperatures in core

o Core-average fuel temperature (Tf_av)

o Core-average coolant temperature (TPb_av) 
- Reactivity contribution

o Coolant density component of reactivity (r_cool)

o Core axial expansion component of reactivity (r_aex)

o Doppler component of reactivity (r_dop)

o Net reactivity (r_net)

o Core radial expansion component of reactivity ( $\mathrm{r}_{-}$rex)

Page 3:

- Reactor power components

o Total reactor power $\left(\mathrm{Q} \_\mathrm{Rx}\right)$

o Decay heat power (Q_dc)

o Fission power (Qfis)

- Reactor power and its prediction

o Reactor power (Q_Rx)

o Relative error of reactor power prediction by extrapolation by kinetics subroutines (E_ex)

- Turbine and compressor work and generator output

o Turbine work (W_Turb)

o Compressor No. 1 power input (W_Comp1)

o Compressor No. 2 power input (W_Comp2)

o Net generator power (W_gen)

o Grid demand (W_grid)

- $\mathrm{CO}_{2}$ flow split

o Fraction of flow which goes through cooler and compressor No. 1 (FS)

\section{Page 4:}

- Compressor stall check

o Stall parameter for Compressor No. 1 (Comp1)

o Stall parameter for Compressor No. 2 (Comp2)

- Turbine and compressor choke check

o Maximum Mach number in Compressor No. 1 (Comp1)

o Maximum Mach number in Compressor No. 2 (Comp2)

o Maximum Mach number in turbine (Turb)

- Shaft speed

o Turbomachinery shaft rotational speed (N_r)

- Valve control actions

o Open area fraction for turbine bypass valve (f_TBP)

o Open area fraction for inventory tank inlet valve (f_INVi)

o Open area fraction for inventory tank outlet valve (f_INVo) 
o Open area fraction for turbine throttle valve (f TIN)

o Open area fraction for Compressor No. 2 throttle valve (f_C2IN)

\section{Page 5:}

- Brayton cycle flow rates

o $\mathrm{CO}_{2}$ flow rates in the Brayton cycle according to Figure 1-3 nodes

- Brayton cycle temperatures

o $\mathrm{CO}_{2}$ temperatures in the Brayton cycle according to Figure 1-3 nodes

- Brayton cycle densities

o $\mathrm{CO}_{2}$ densities in the Brayton cycle according to Figure 1-3 nodes

- Brayton cycle pressures

o $\mathrm{CO}_{2}$ pressures in the Brayton cycle according to Figure 1-3 nodes

\subsubsection{Full Power Operation - Steady-State and Stability Test}

Before any disturbances were introduced into the system, the code was checked for stability and prediction of the steady-state parameters. For this purpose, a transient run was initiated with the grid load set to $100 \%$ for all times. The plant control system has to maintain stable operation at full power; the system parameters have to be constant close to the steady-state values. Some differences from steady-state values as well as initial stabilization of the parameters are expected, since the assumptions made for the dynamics model are not the same as for the steady-state model. These differences are acceptable as long as they are small.

Since no disturbance is introduced into the system, the only changes at the beginning of the transient are: i) switching to the reactor power calculations by the kinetics subroutines; ii) start of the shaft dynamics calculations; and iii) plant control system actions.

Figure 1-11 shows the results of system operation at the full power simulation. All calculated system parameters are close to the steady-state values (perhaps, the biggest difference is in reactor power which stabilizes at $399.4 \mathrm{MW}$ vs. $400 \mathrm{MW}$ at steady-state about a $0.15 \%$ difference). Also, all parameters are stable for at least $1000 \mathrm{~s}$ (the total simulation time). The Brayton cycle control system adds some $\mathrm{CO}_{2}$ inventory to the cycle and opens the turbine bypass valve slightly. Some bypass flow is required for stable operation of the control system (to avoid constant switching between turbine bypass and inventory controls). These changes are too small to affect the cycle, as shown in Figure 1-11, but are enough for stable operation. 


\subsubsection{Plant Response to Step Changes in Load}

The sudden changes (occurring over $0.1 \mathrm{~s}$ ) of the generator load have been introduced into the system to simulate step changes in grid load. Figure 1-12 and Figure 1-13 show the system response to $1 \%$ increase and decrease in grid load, respectively. Figure 1-14 shows the system response to a 10\% step decrease in load. All responses are satisfactory; the control system can safely handle the changes and the system operates stably at the new load levels.

\subsubsection{Plant Response to Linear Changes in Load}

Linear changes in grid demand at $3 \% / \mathrm{min}$ are simulated. Several runs are made to show that the system can safely accommodate linear changes in grid demand at this rate from full power down to any power level.

Figure 1-15 shows the system response to a linear change from full power to $50 \%$ load, operation at $50 \%$ load, and return to full power. The system response is satisfactory. However, there is a small amount of non-steady behavior at the point of return to full power when the inventory control has to be used in addition to the turbine bypass control for the shaft speed regulation. The non-steady-behavior is overcome in a short time and does not cause any long-term consequences.

Figure 1-16 shows the system response to a full-range (from $100 \%$ to $0 \%$ ) load change. It demonstrates operation and interaction between all Brayton cycle control mechanisms. It also demonstrates that the plant can be controlled over the full range of grid changes without any active control on the reactor side (no external reactivity control is programmed in the code). Thus, autonomous reactor control is demonstrated. 

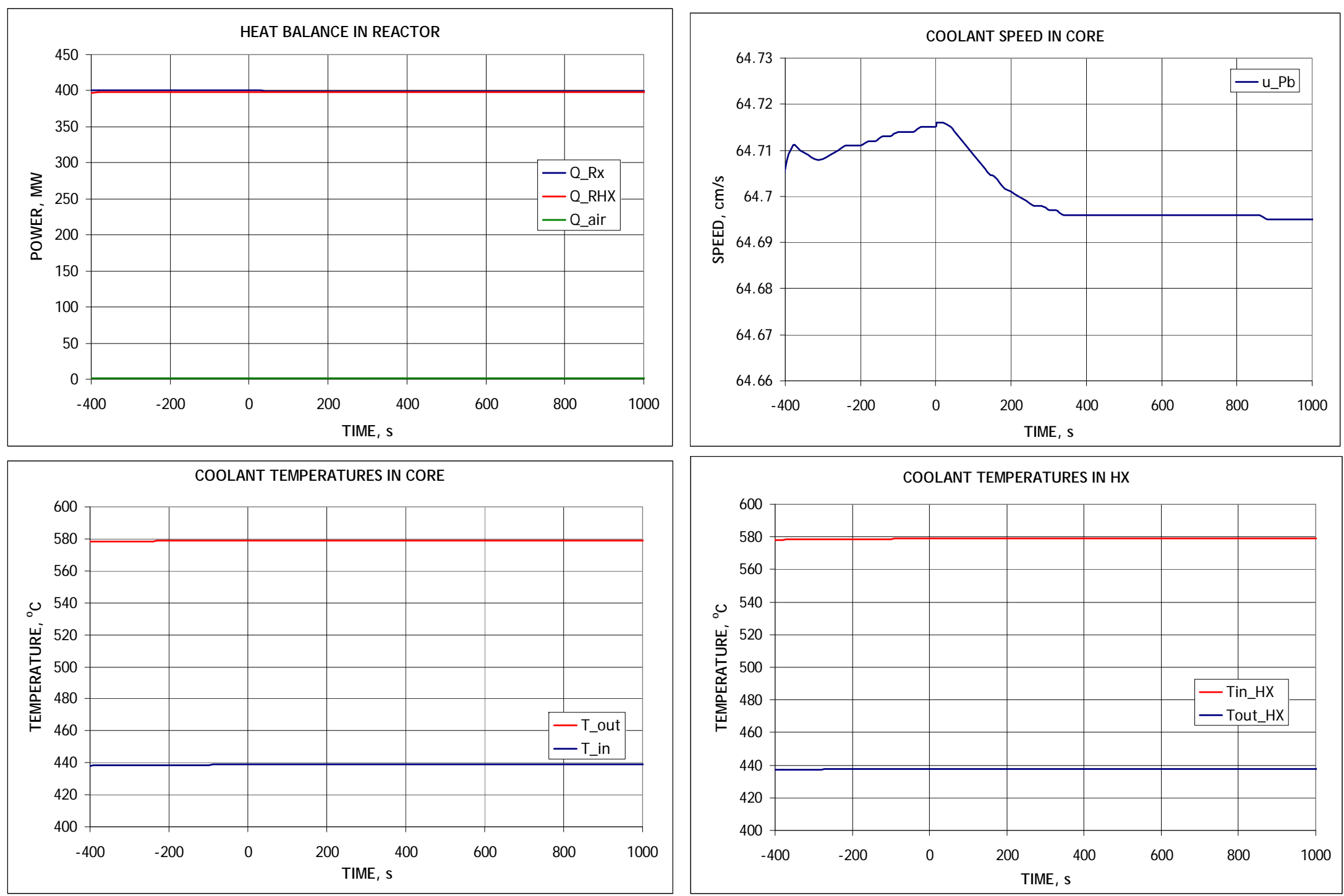

Figure 1-11. Operation at Full Power. 

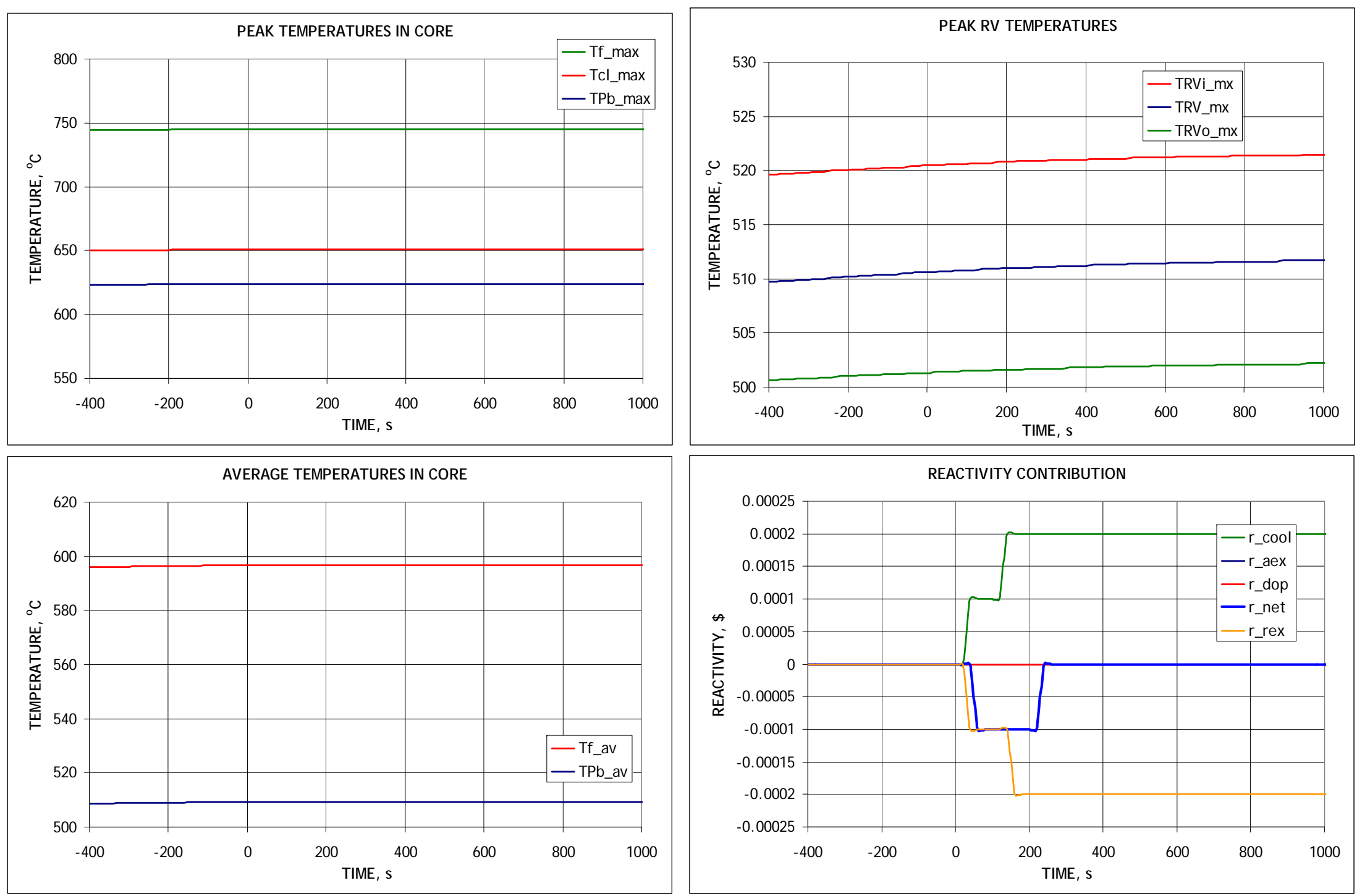

Figure 1-11. Operation at Full Power (2). 

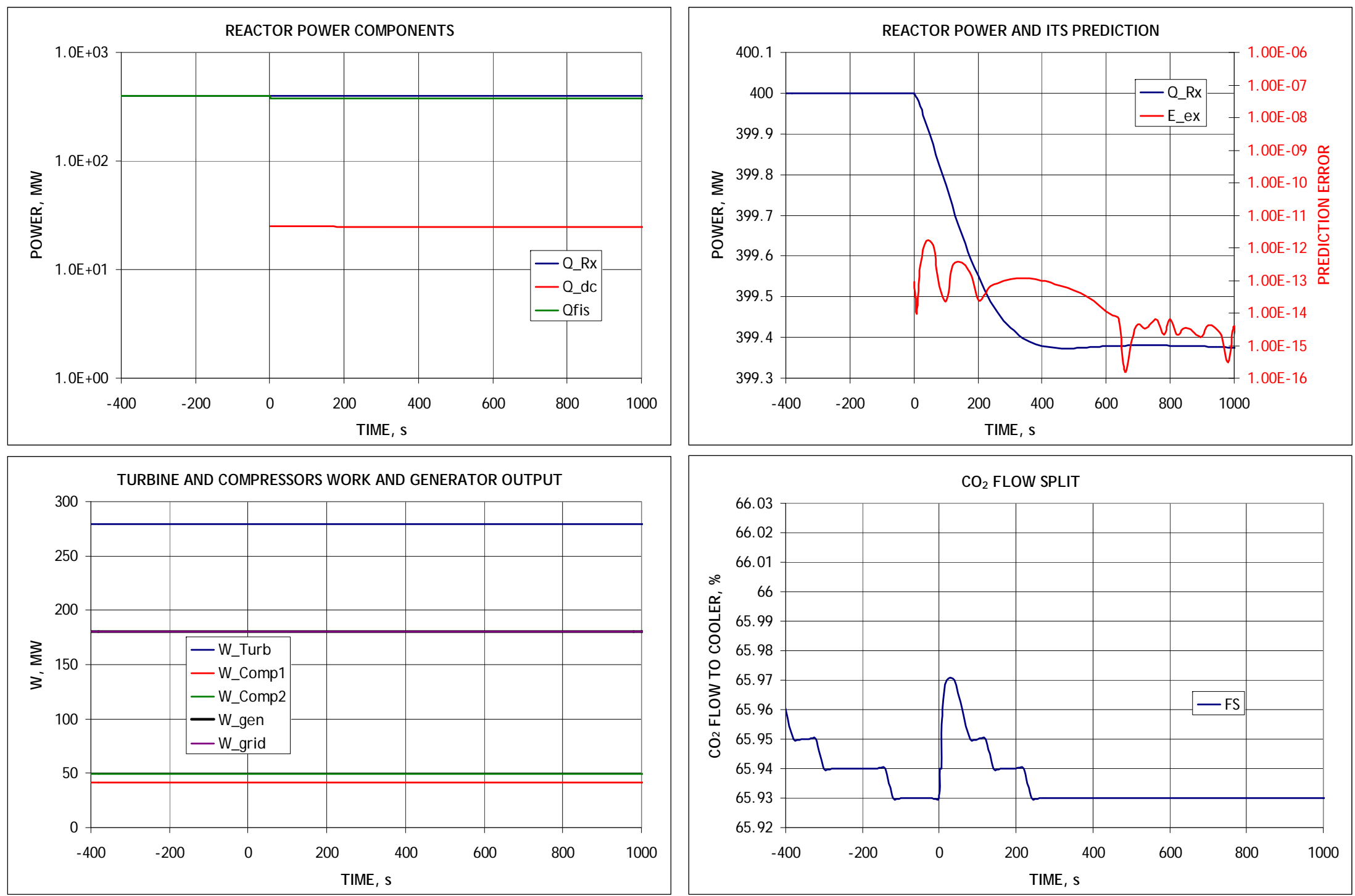

Figure 1-11. Operation at Full Power (3). 

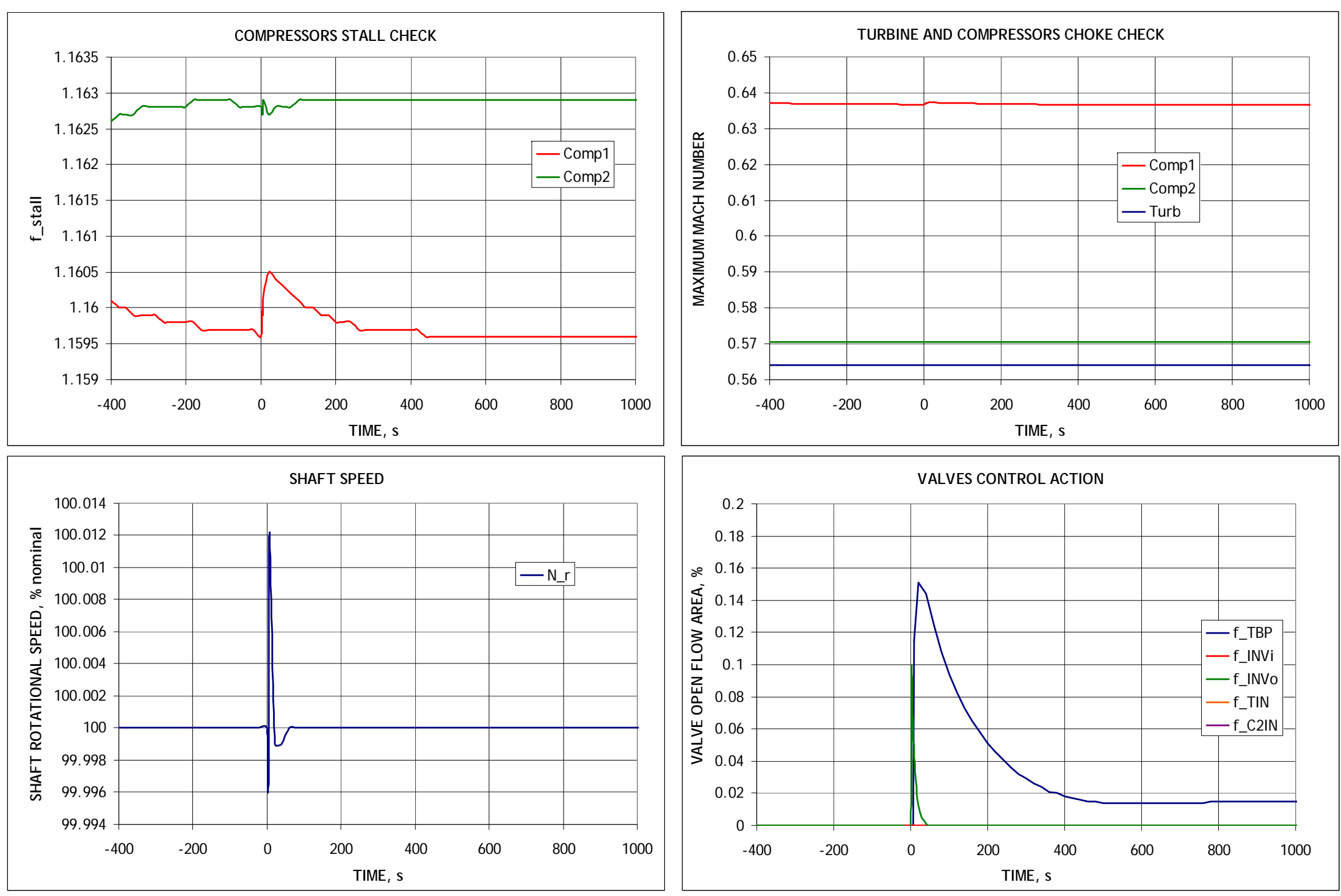

Figure 1-11. Operation at Full Power (4). 

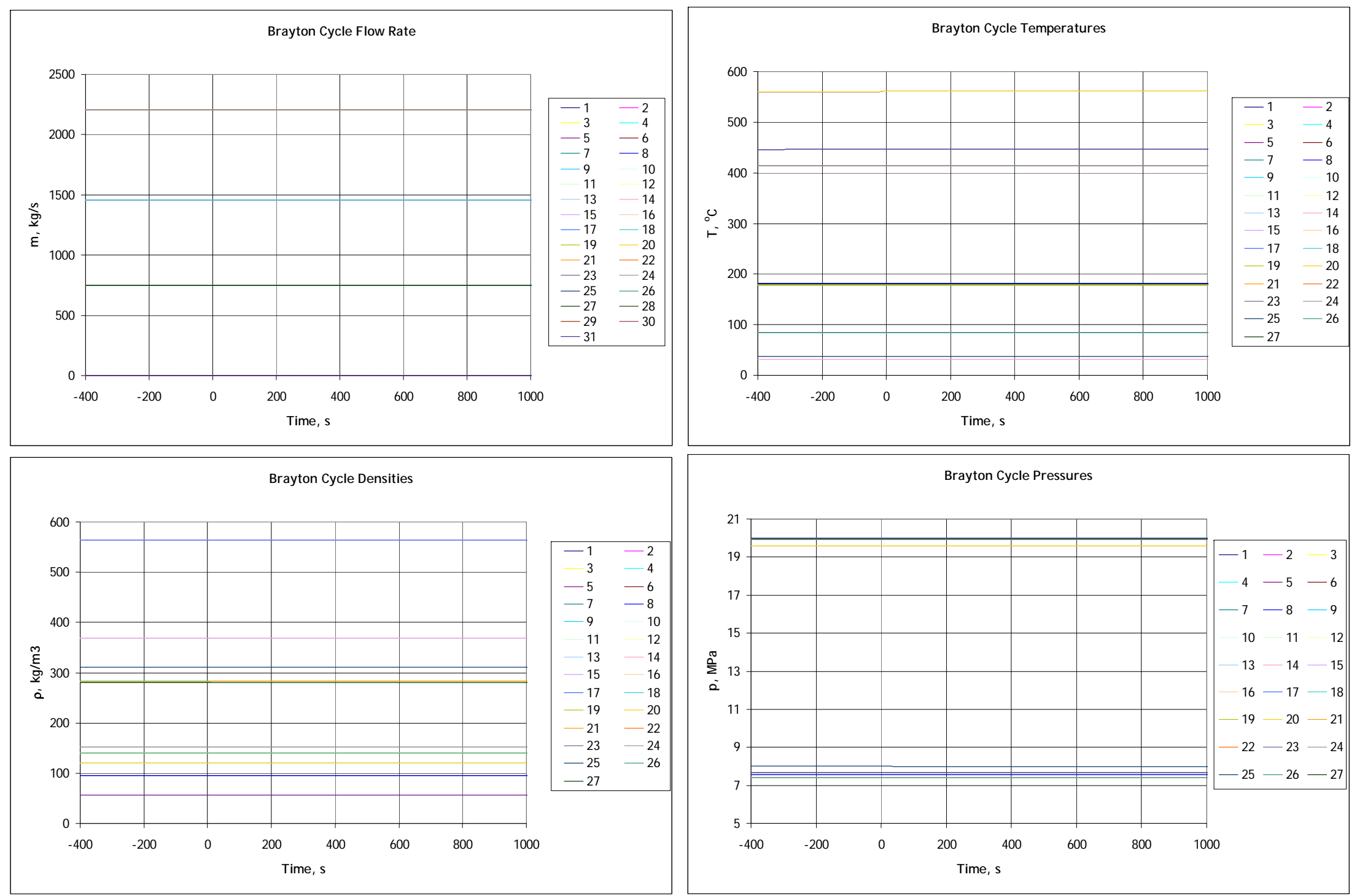

Figure 1-11. Operation at Full Power (5). 

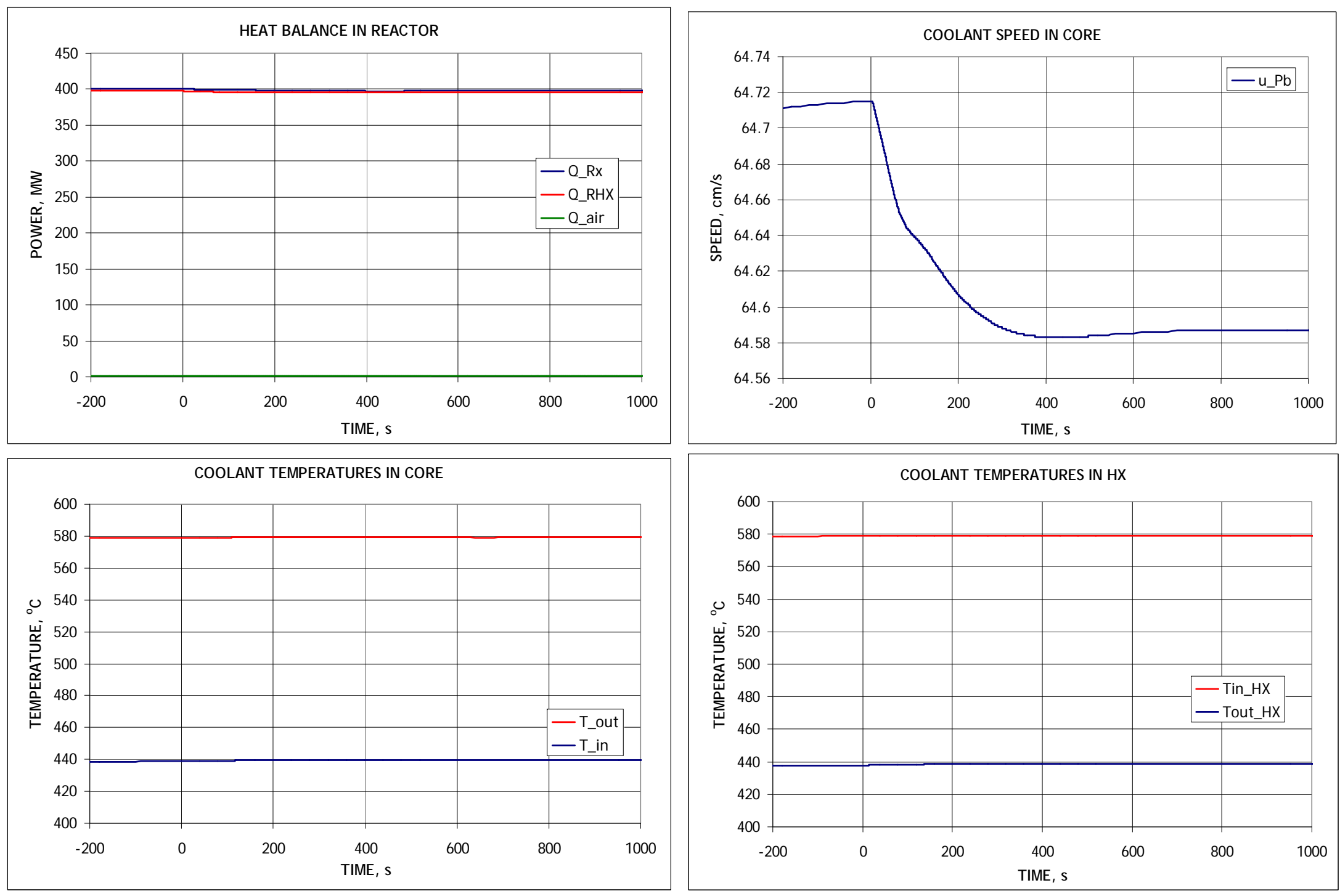

Figure 1-12. 1\% Step Decrease in Load. 

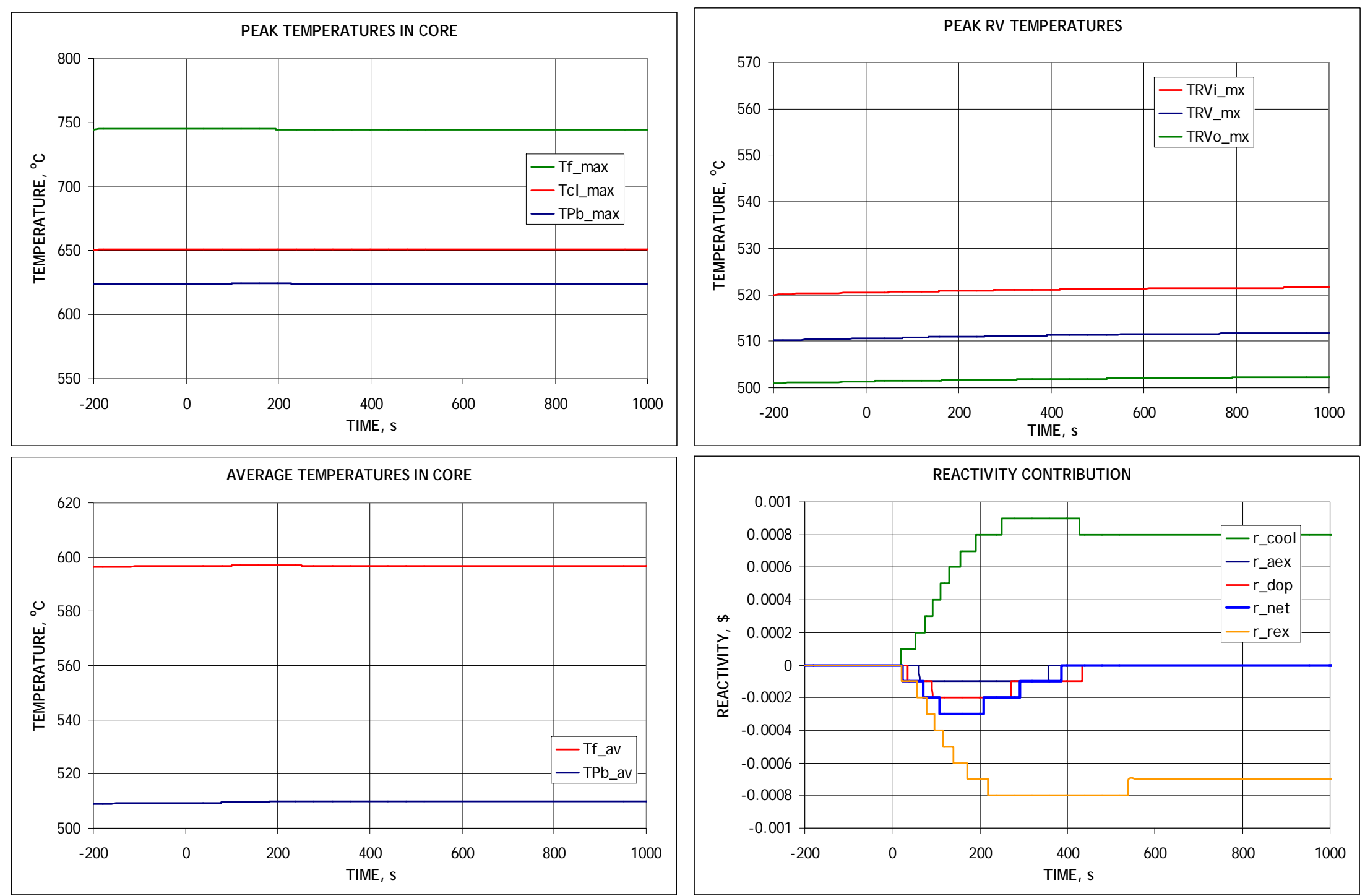

Figure 1-12. 1\% Step Decrease in Load (2). 

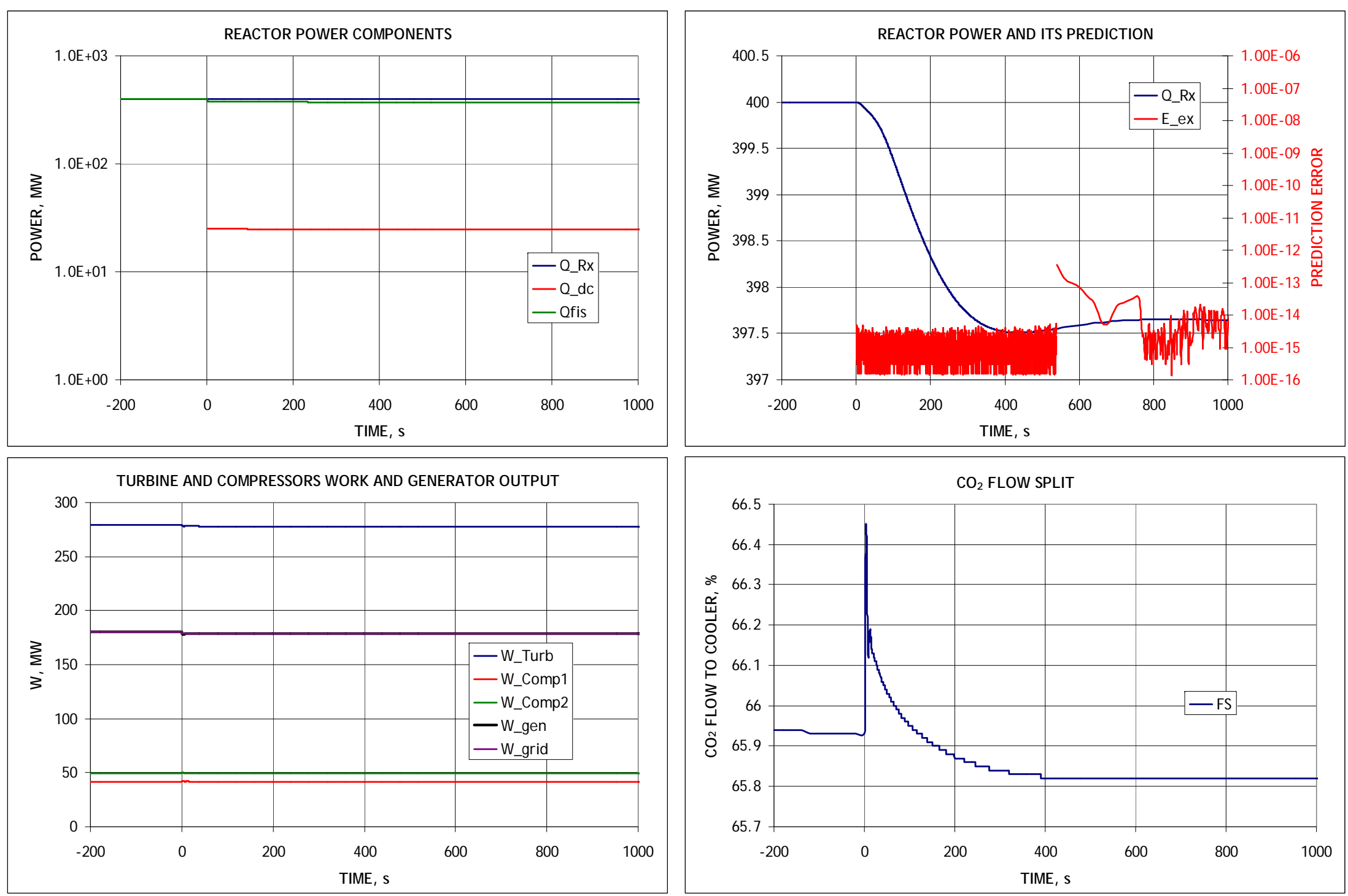

Figure 1-12. 1\% Step Decrease in Load (3). 

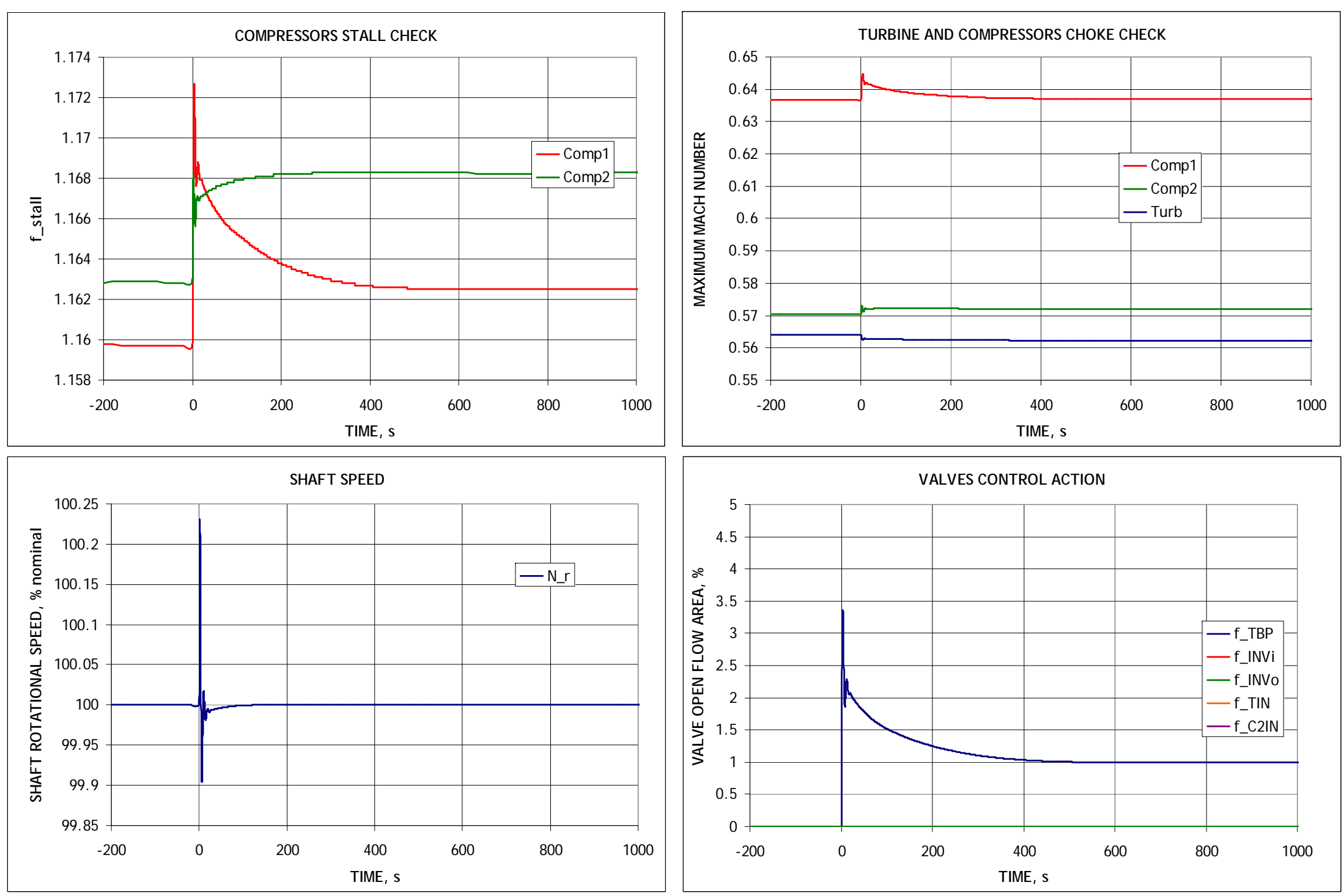

Figure 1-12. 1\% Step Decrease in Load (4). 

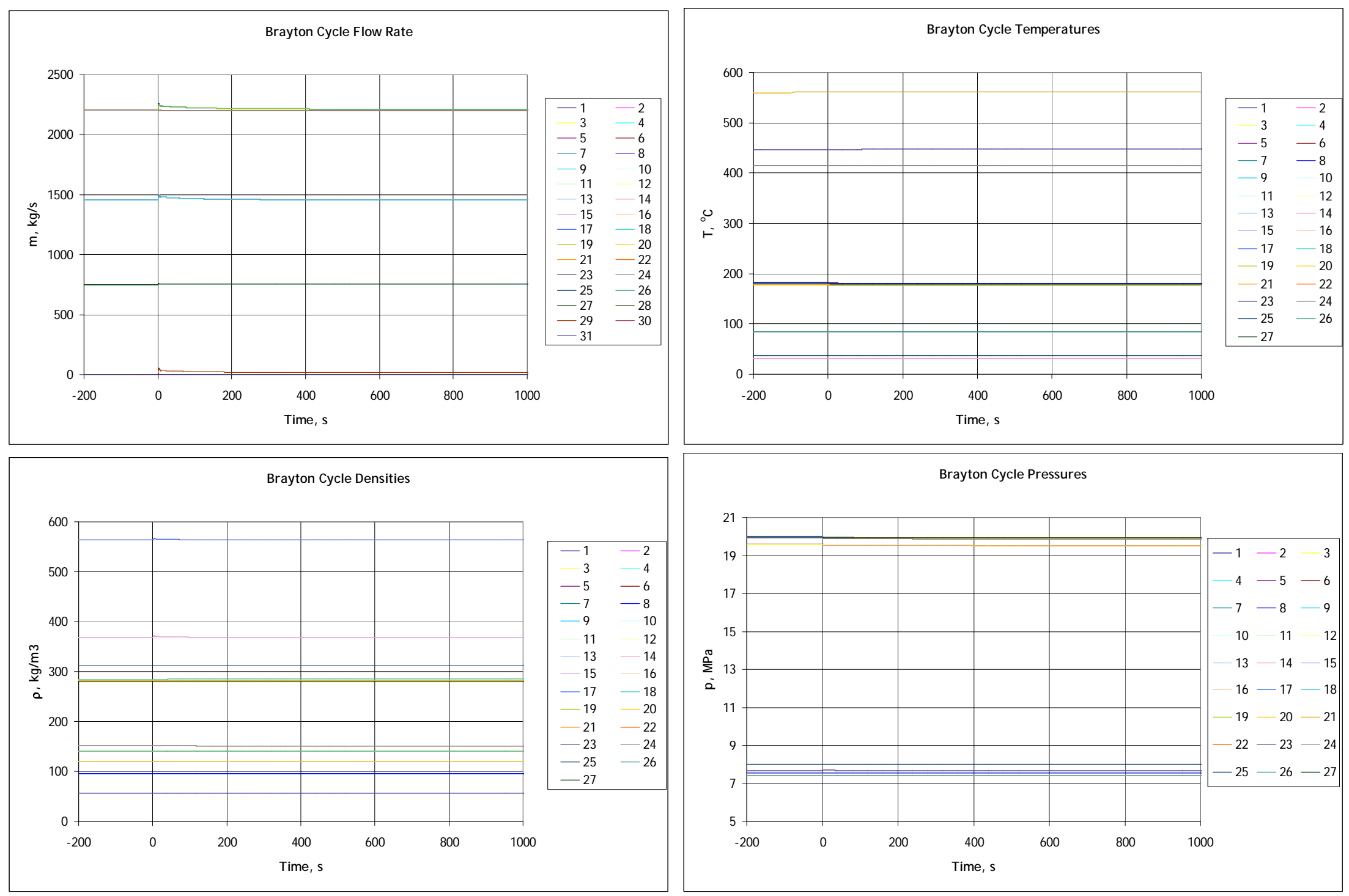

Figure 1-12. 1\% Step Decrease in Load (5). 

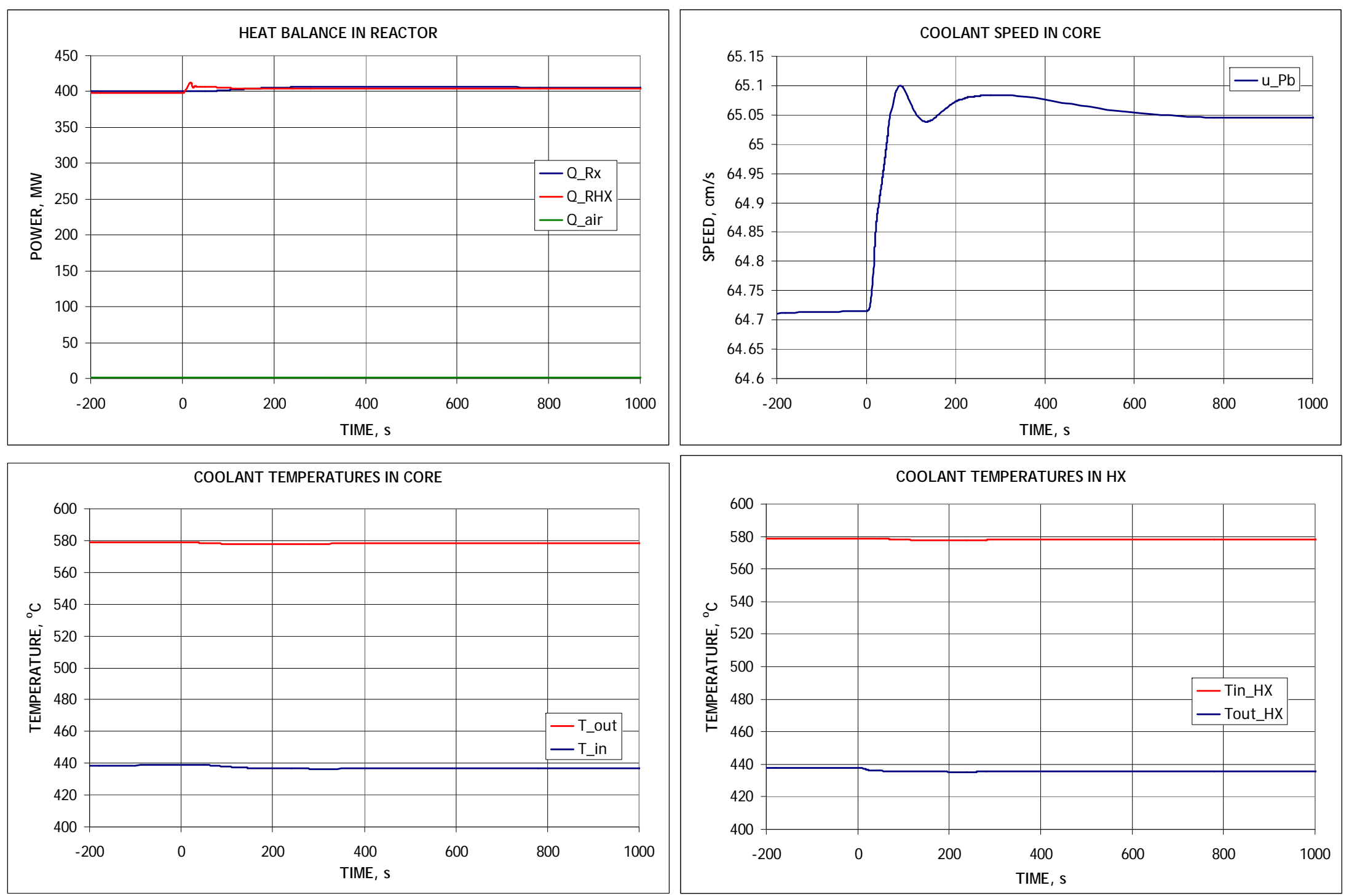

Figure 1-13. 1\% Step Increase in Load. 

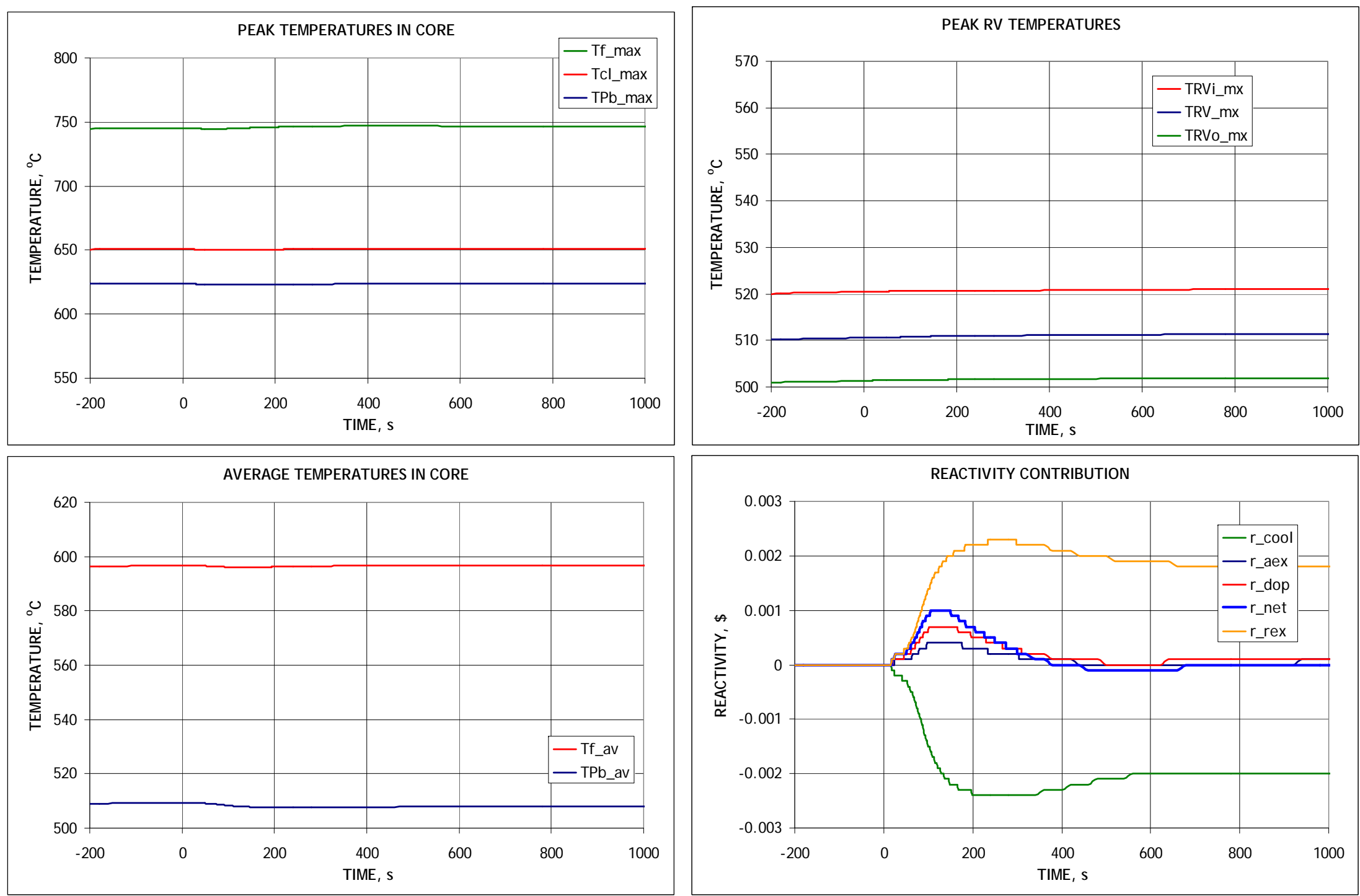

Figure 1-13. 1\% Step Increase in Load (2). 

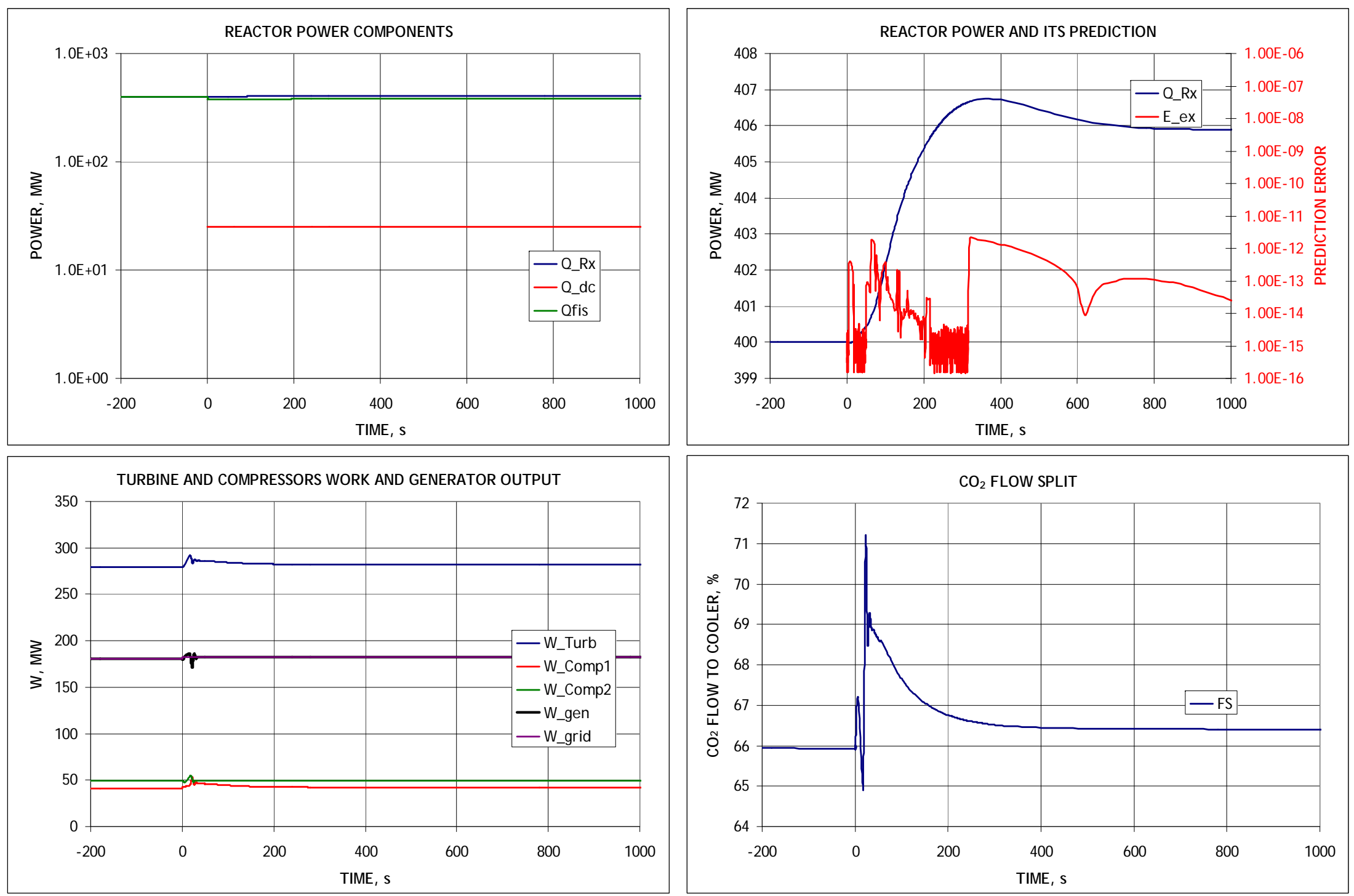

Figure 1-13. 1\% Step Increase in Load (3). 

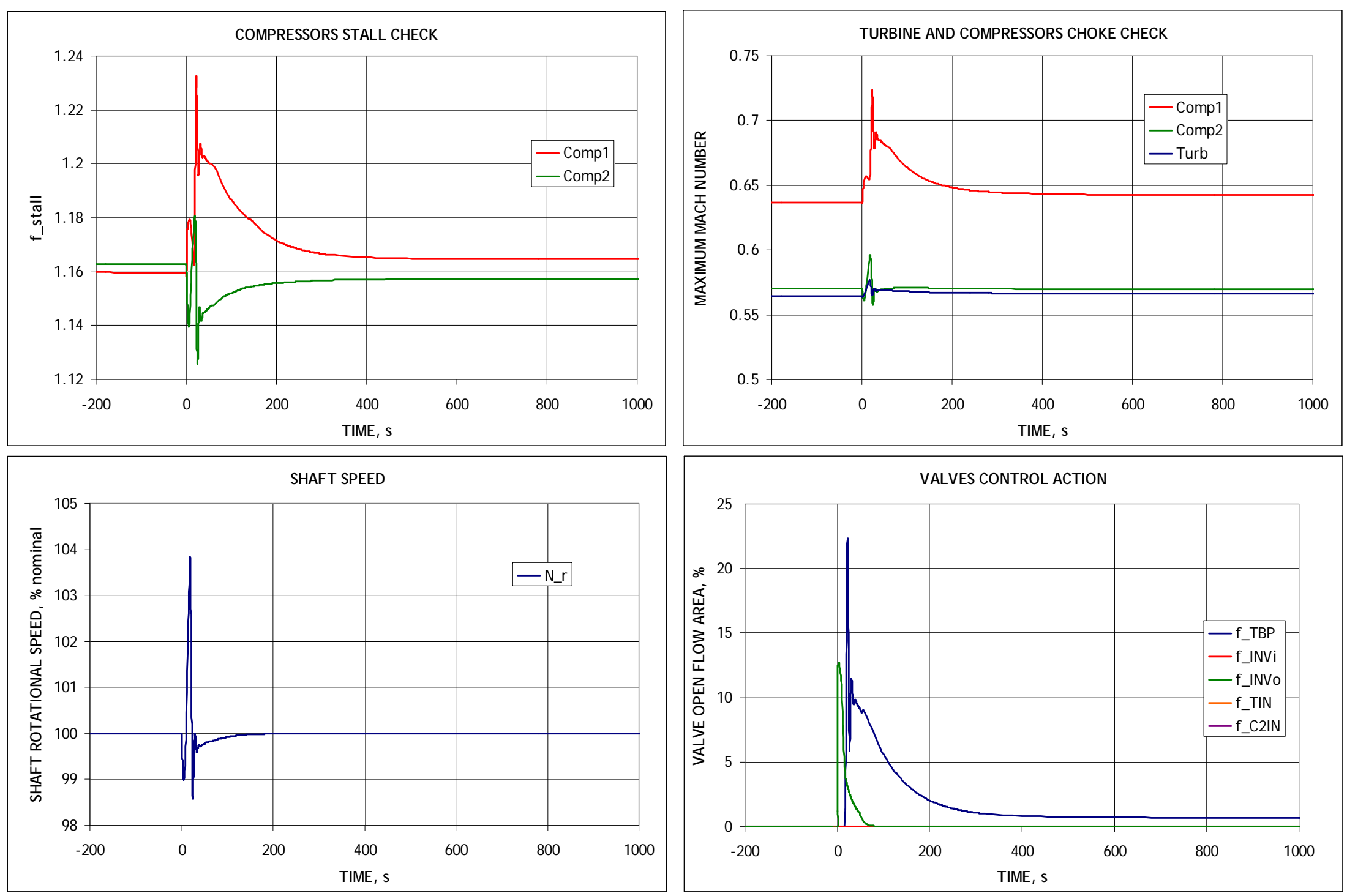

Figure 1-13. 1\% Step Increase in Load (4). 

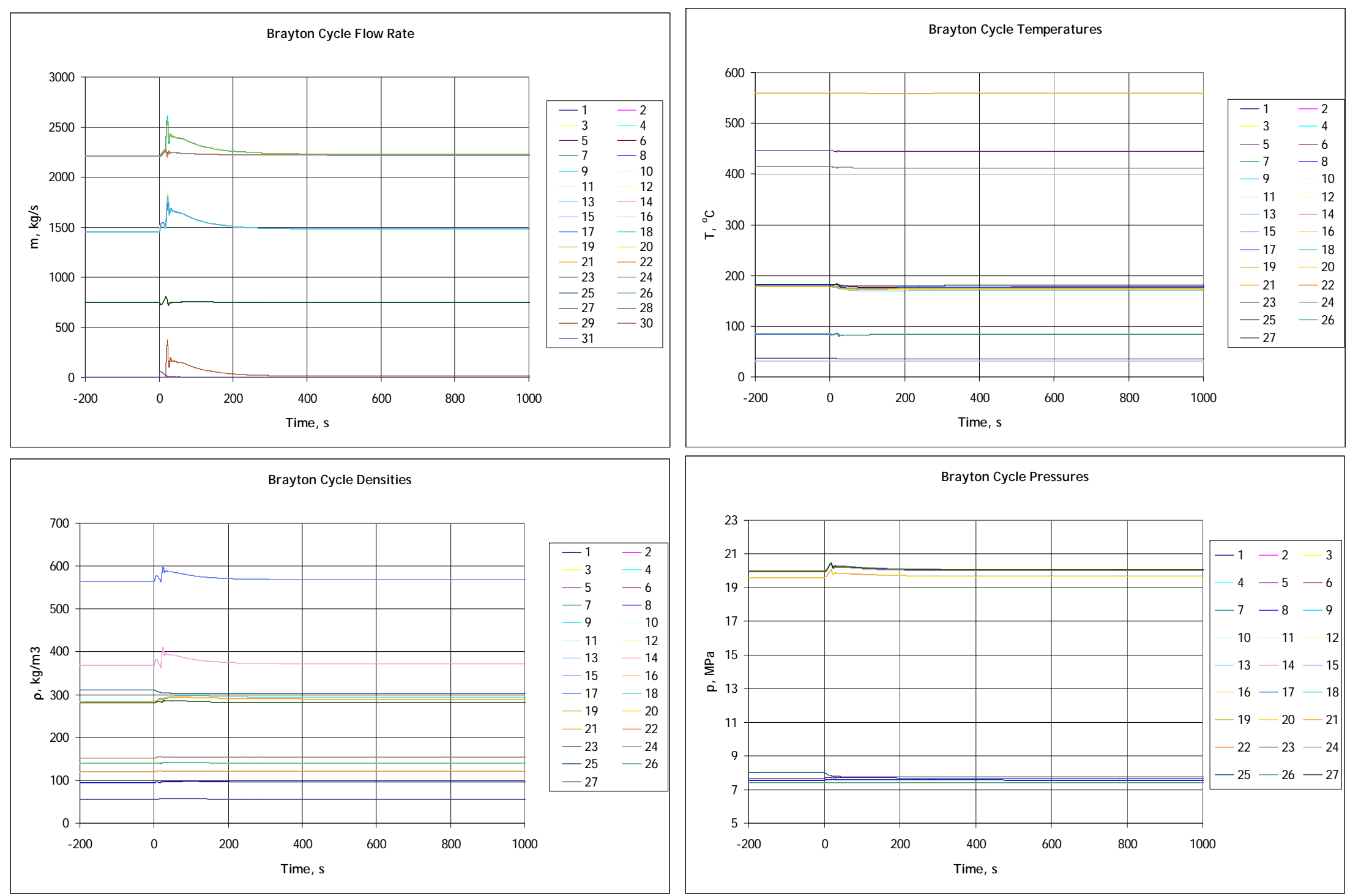

Figure 1-13. 1\% Step Increase in Load (5). 

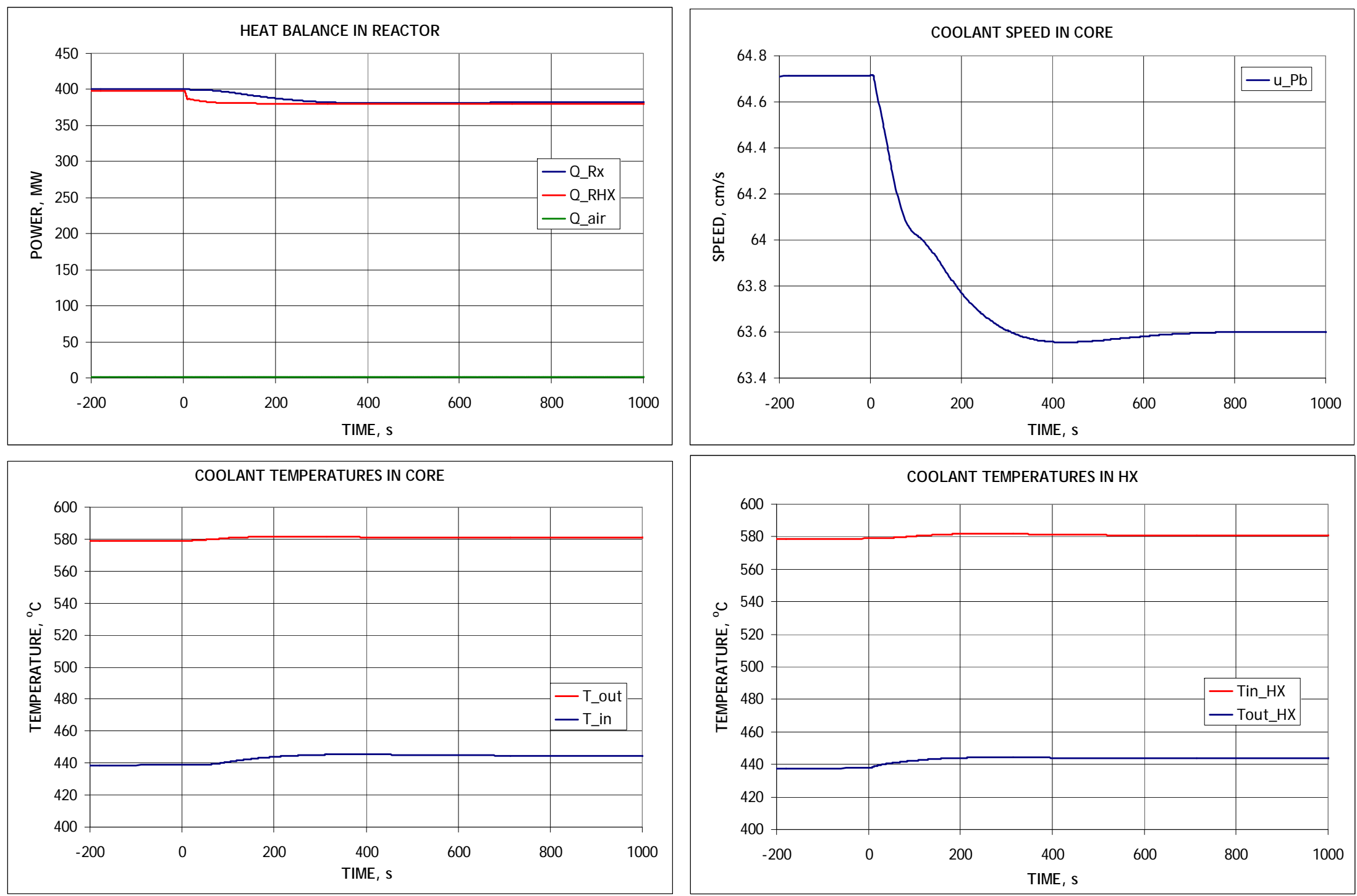

Figure 1-14. 10\% Step Decrease in Load. 

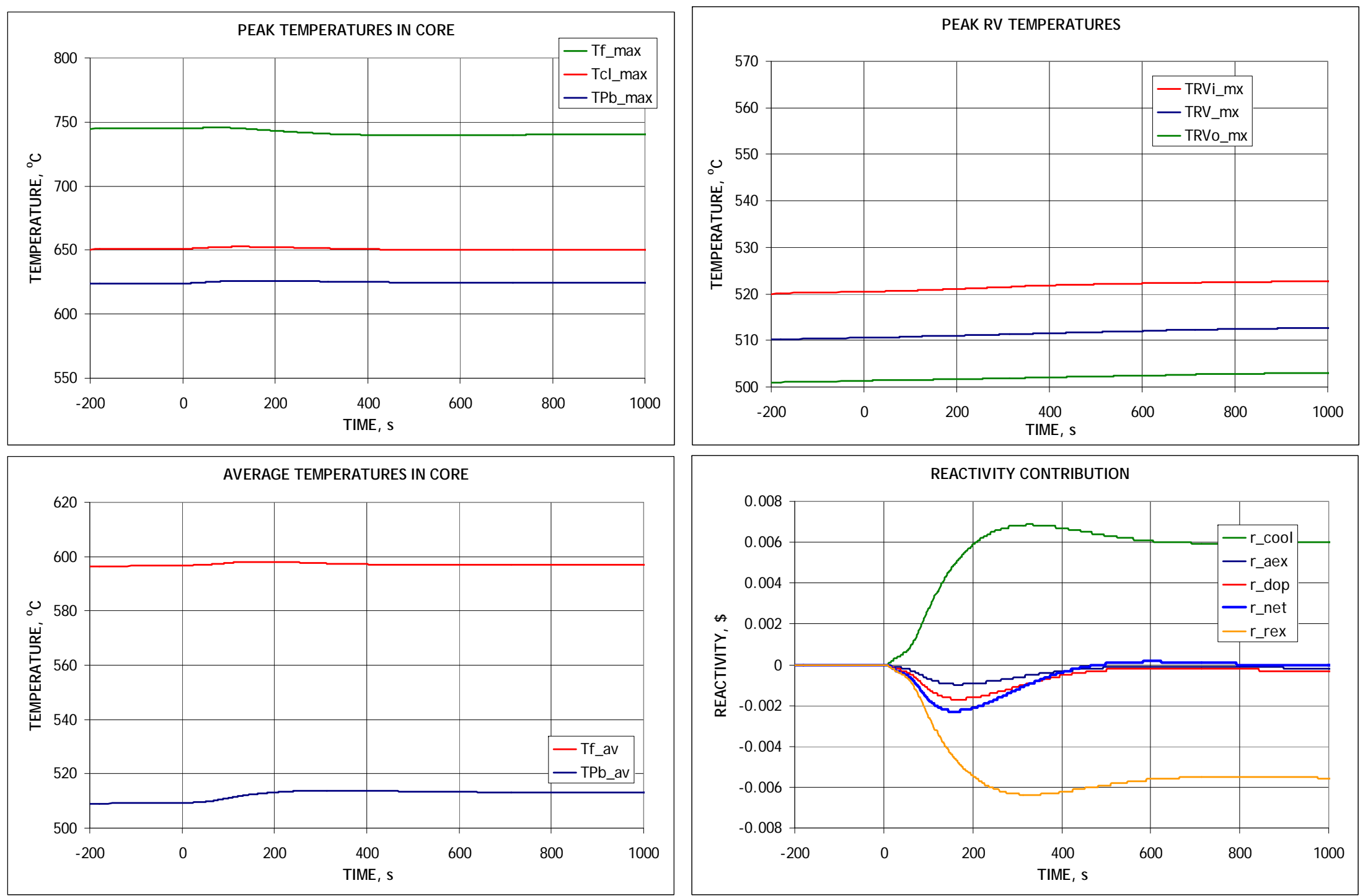

Figure 1-14. 10\% Step Decrease in Load (2). 

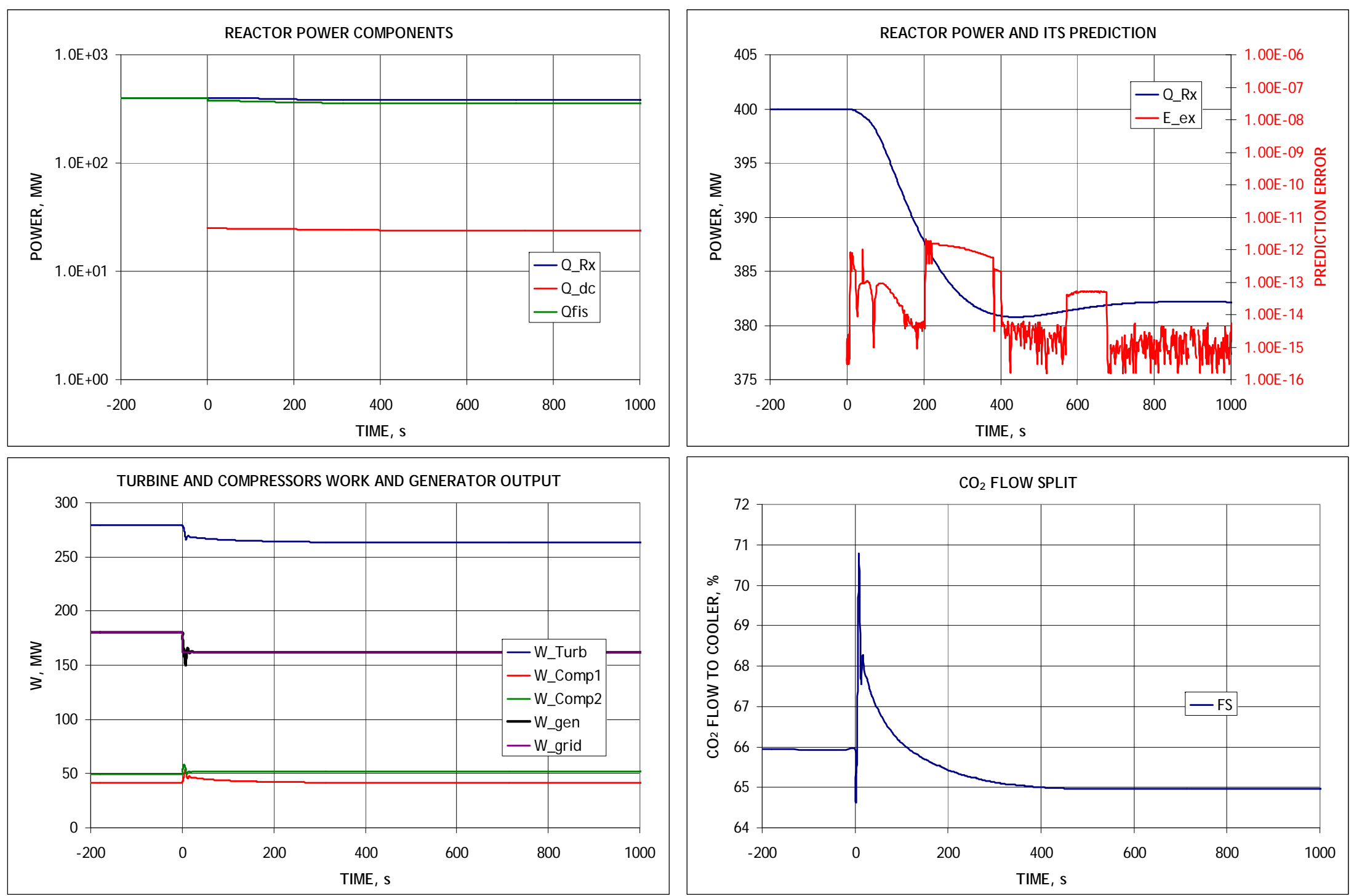

Figure 1-14. 10\% Step Decrease in Load (3). 

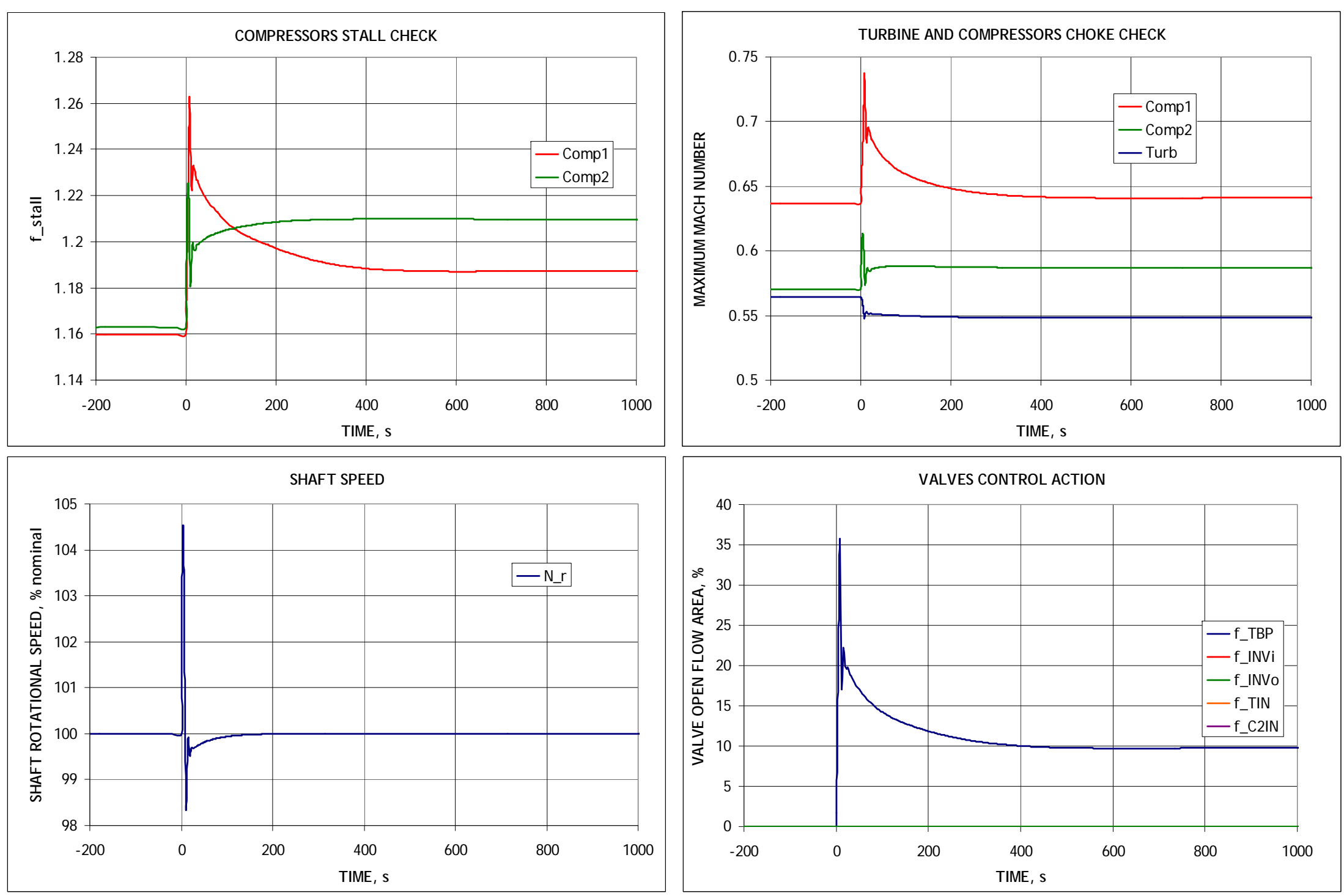

Figure 1-14. 10\% Step Decrease in Load (4). 

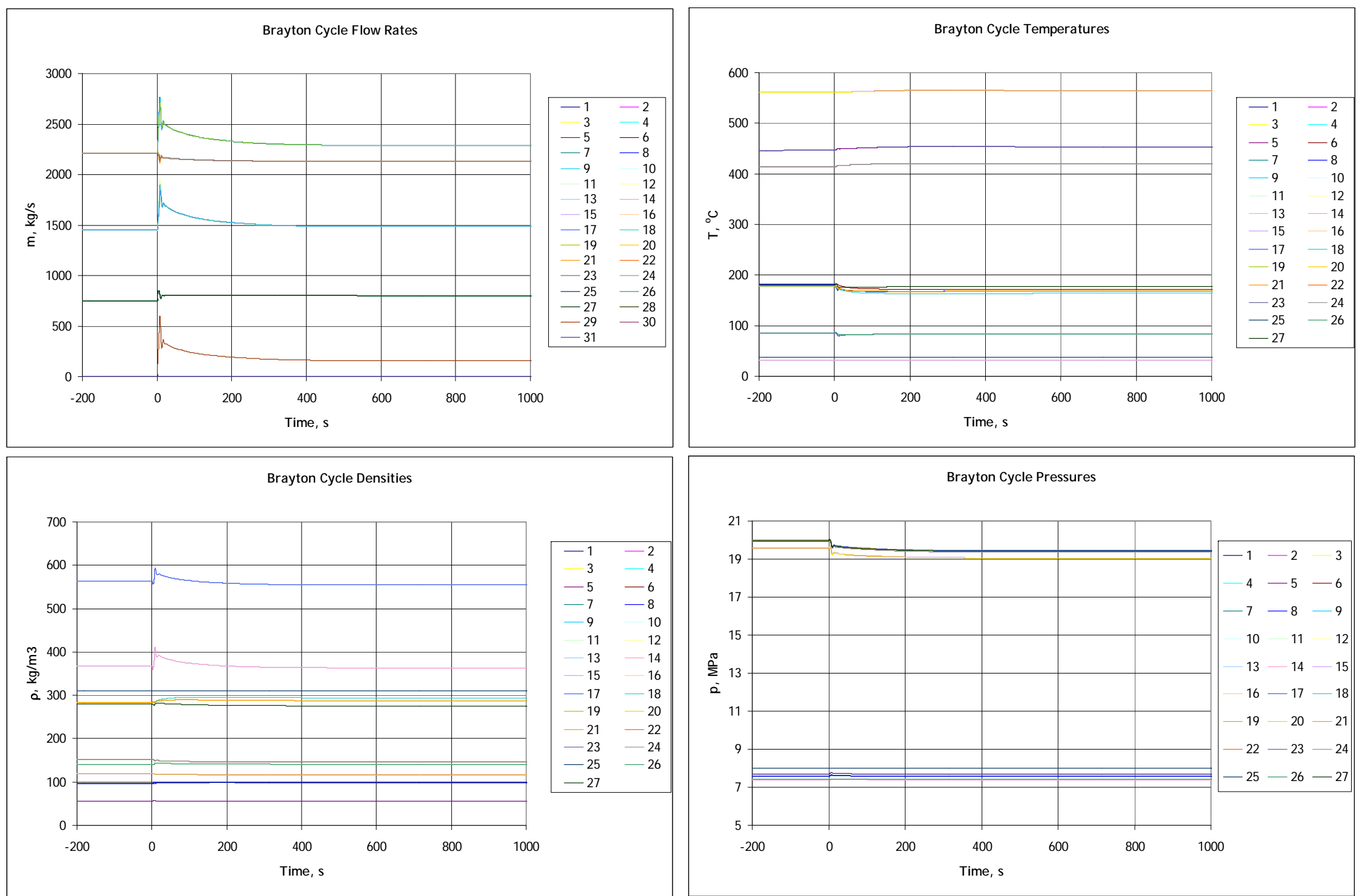

Figure 1-14. 10\% Step Decrease in Load (5). 

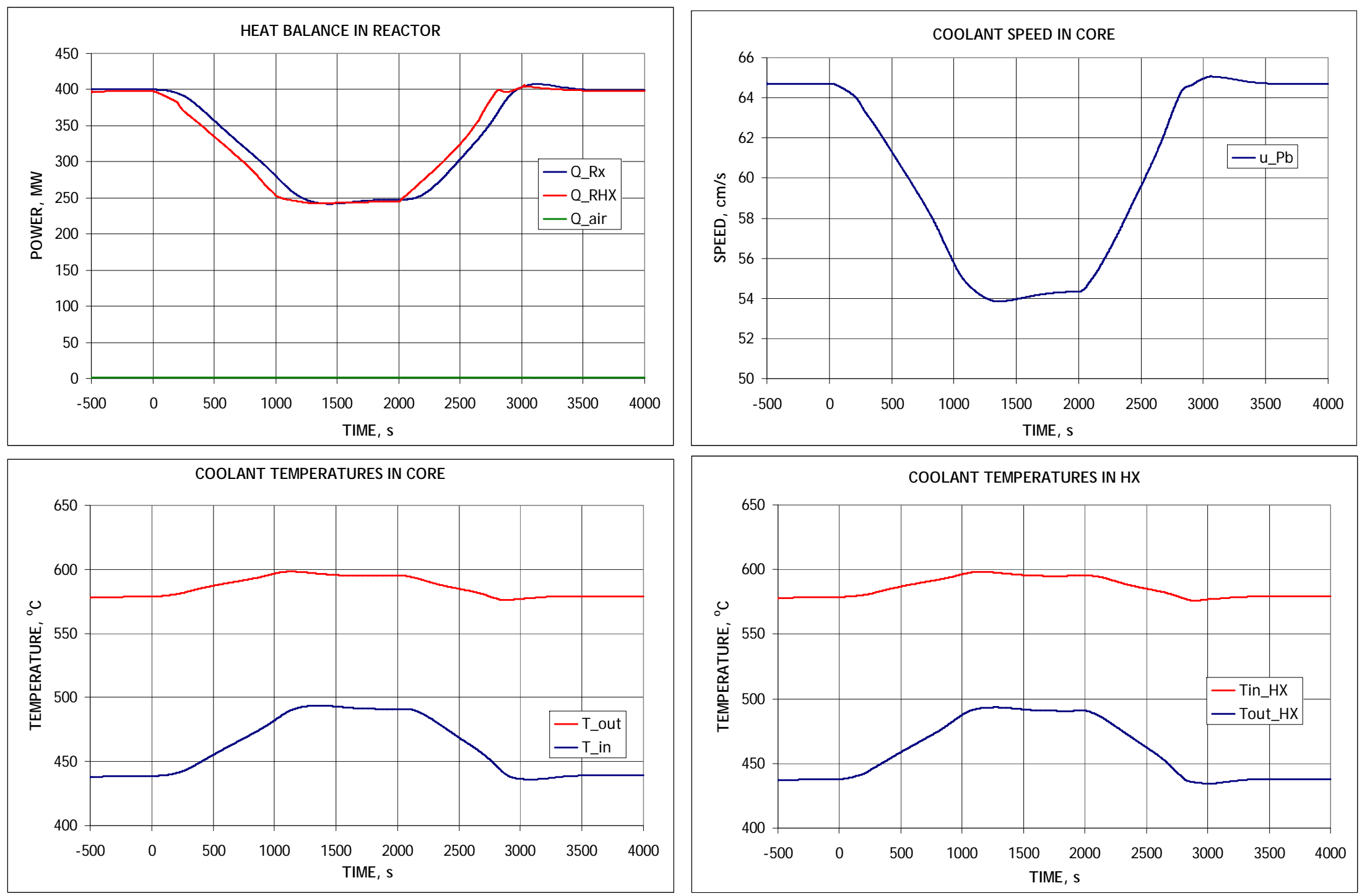

Figure 1-15. 50\% Linear Decrease in Load with Return to Full Load. 

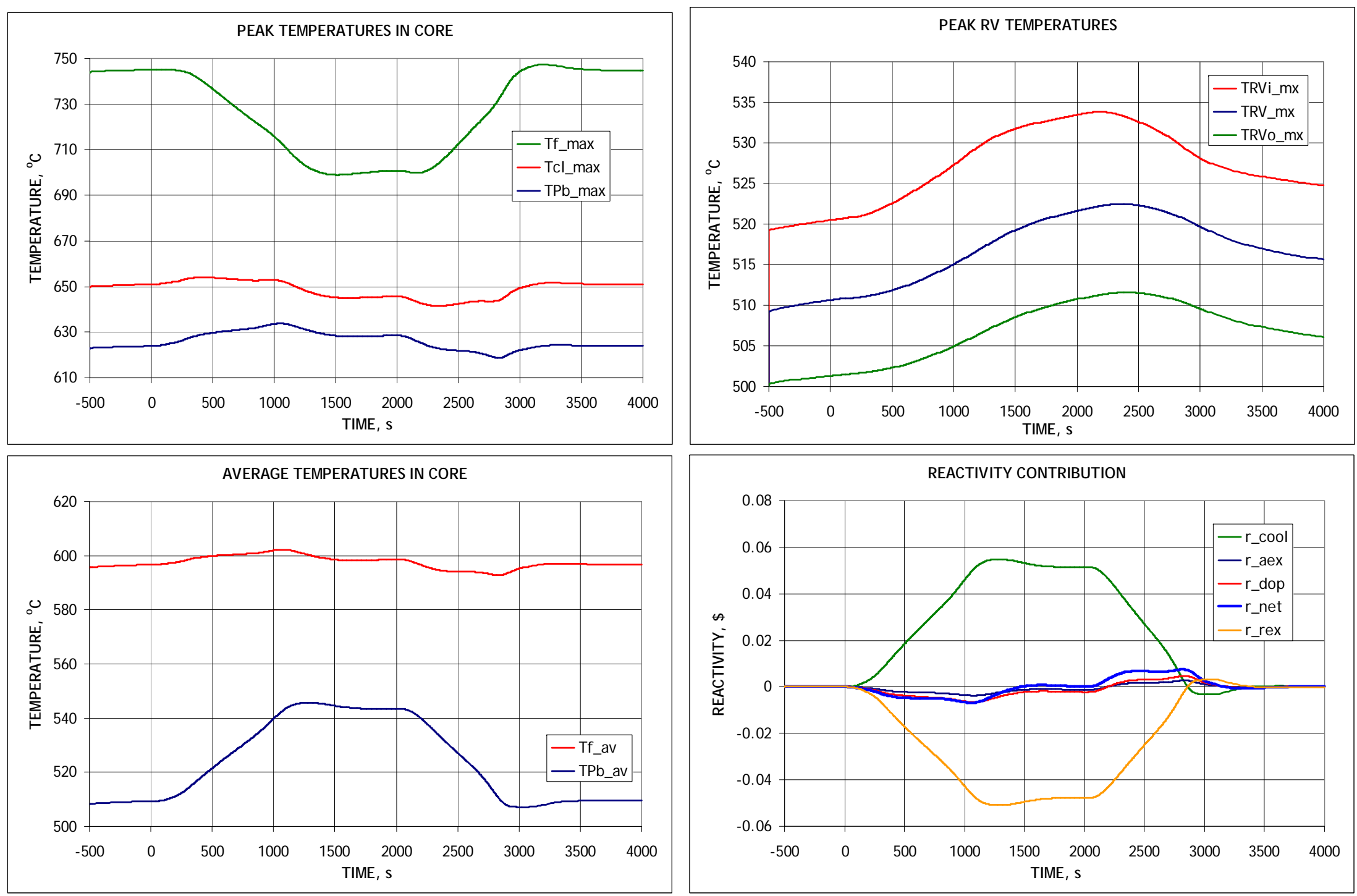

Figure 1-15. 50\% Linear Decrease in Load with Return to Full Load (2). 

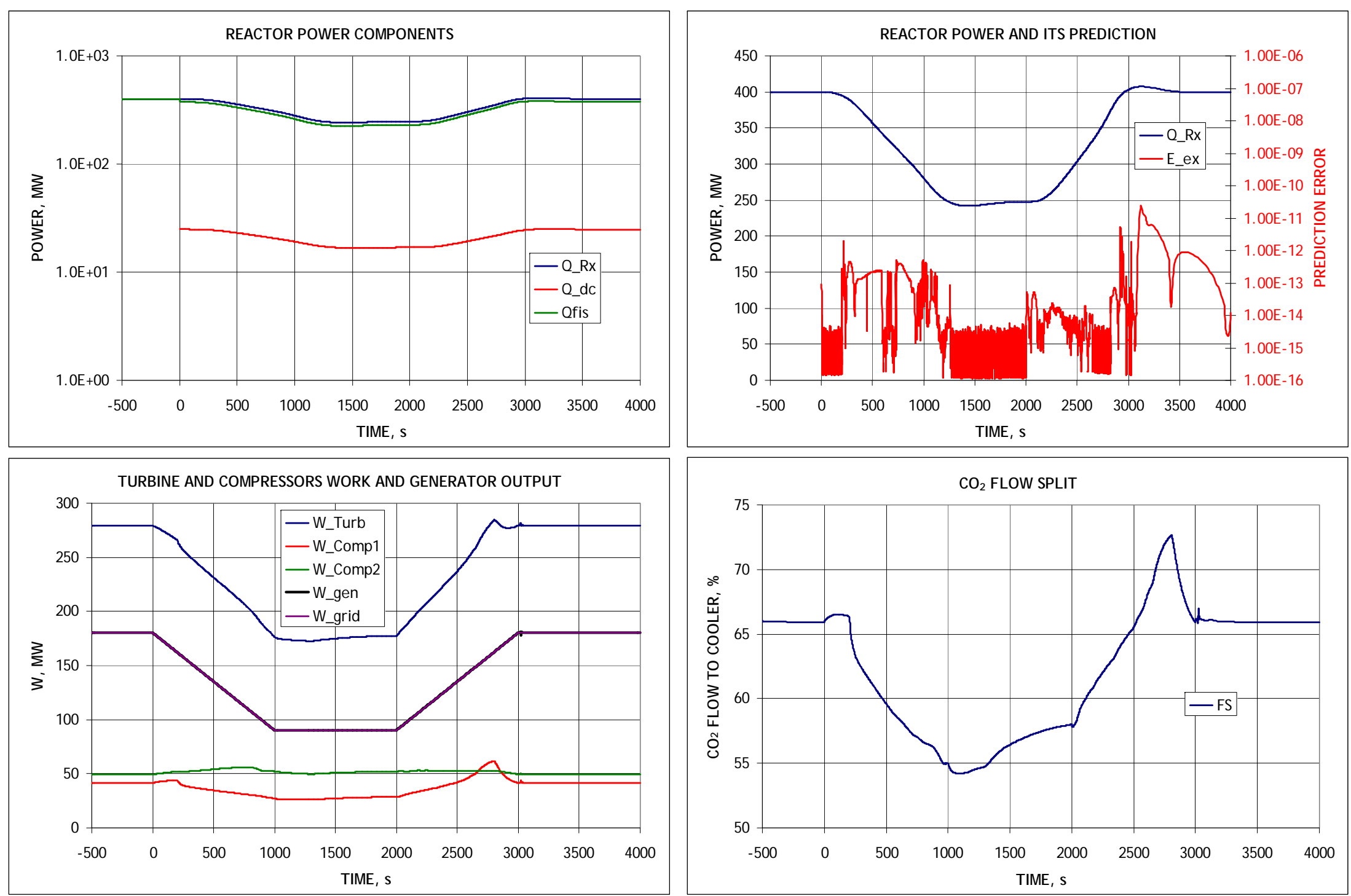

Figure 1-15. 50\% Linear Decrease in Load with Return to Full Load (3). 

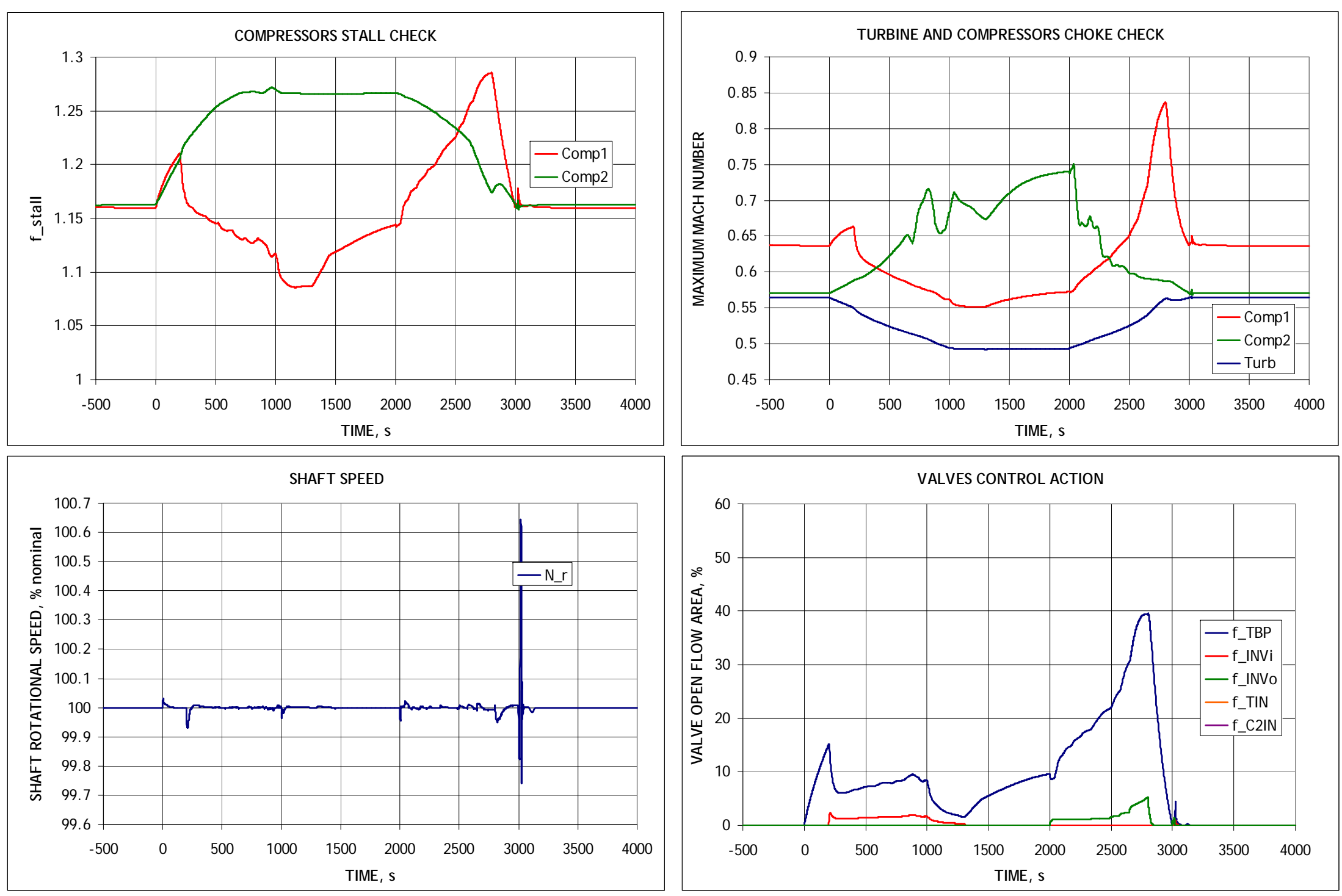

Figure 1-15. 50\% Linear Decrease in Load with Return to Full Load (4). 

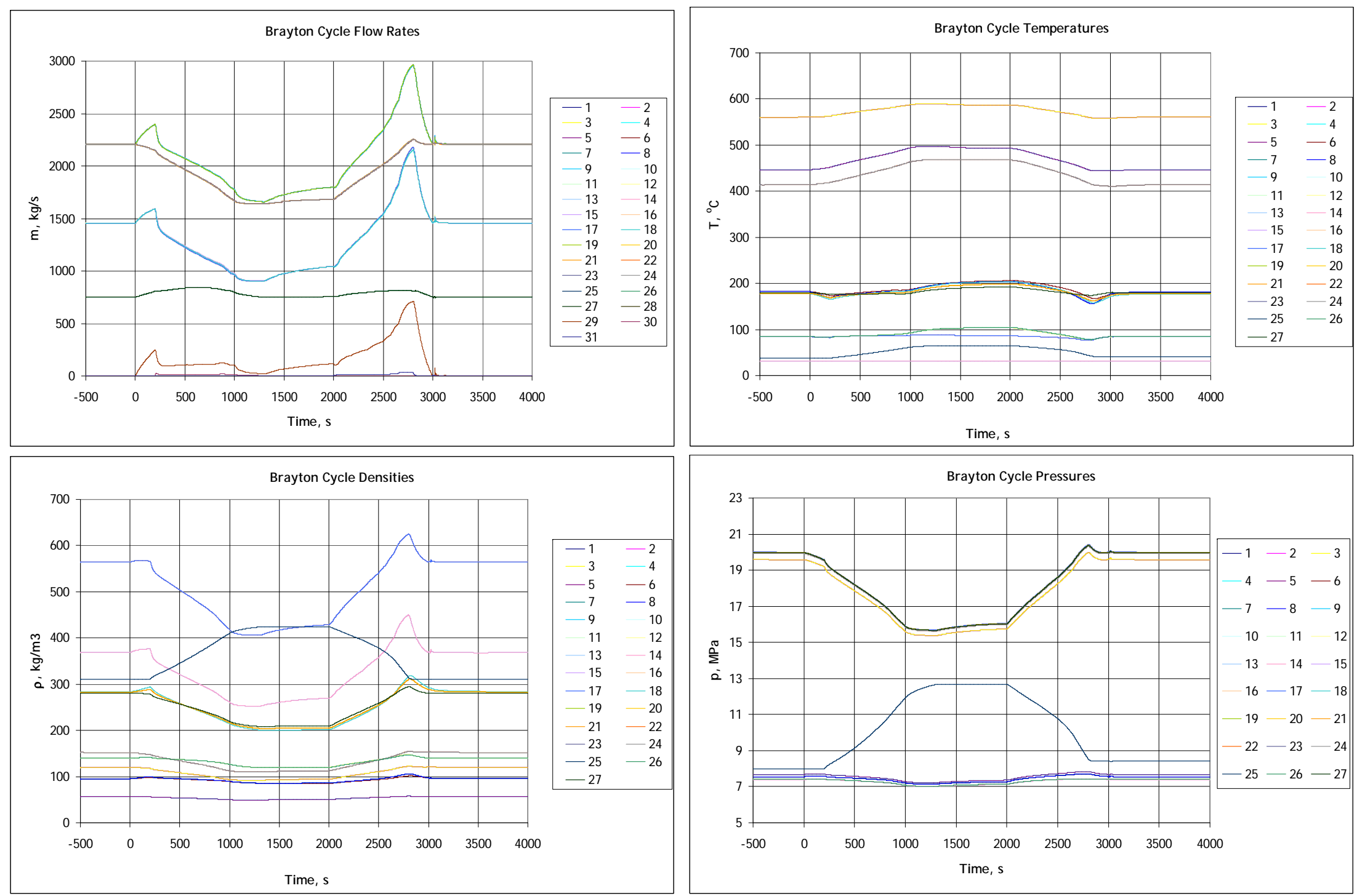

Figure 1-15. 50\% Linear Decrease in Load with Return to Full Load (5). 

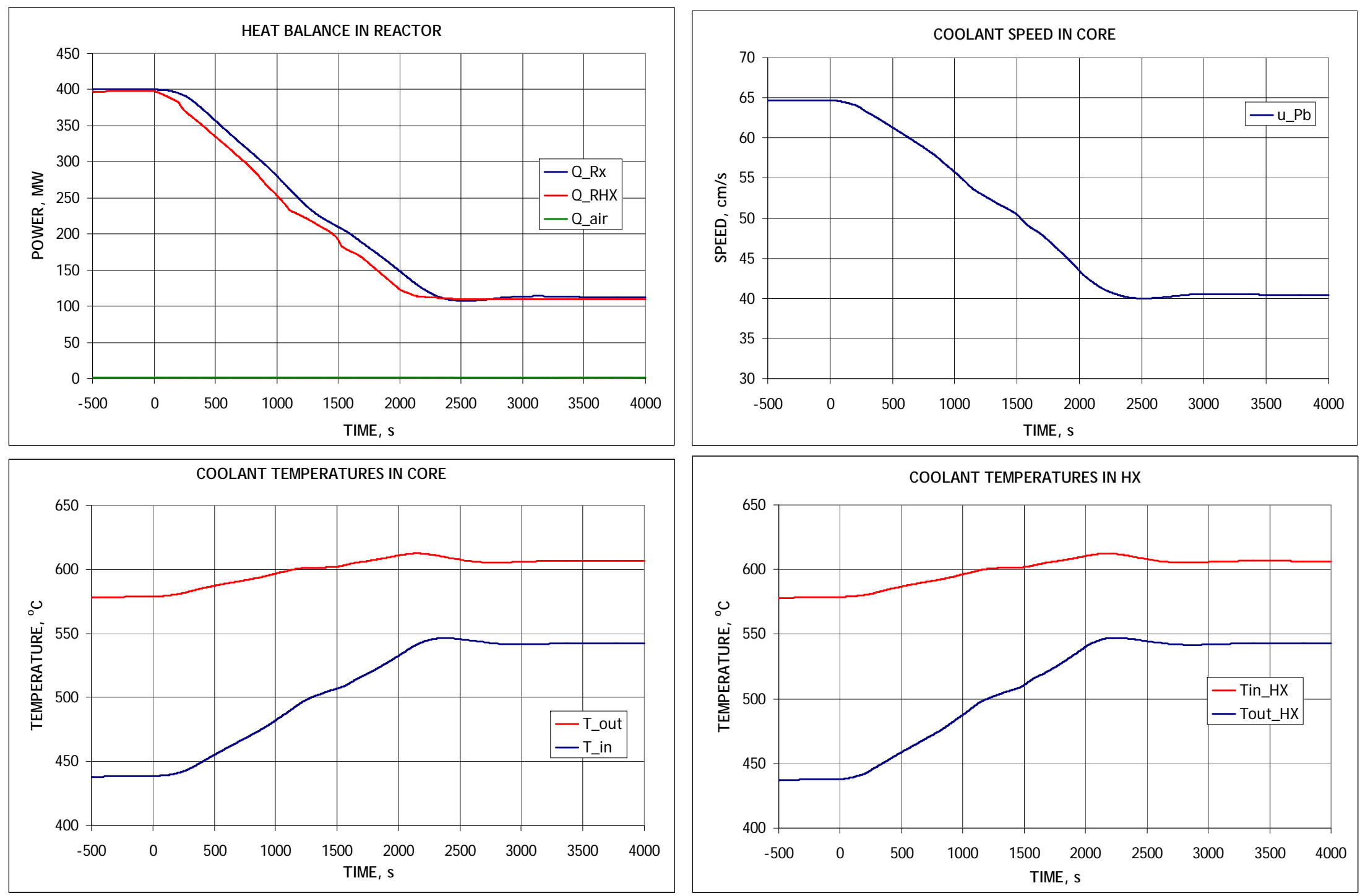

Figure 1-16. 100\% Linear Decrease in Load. 

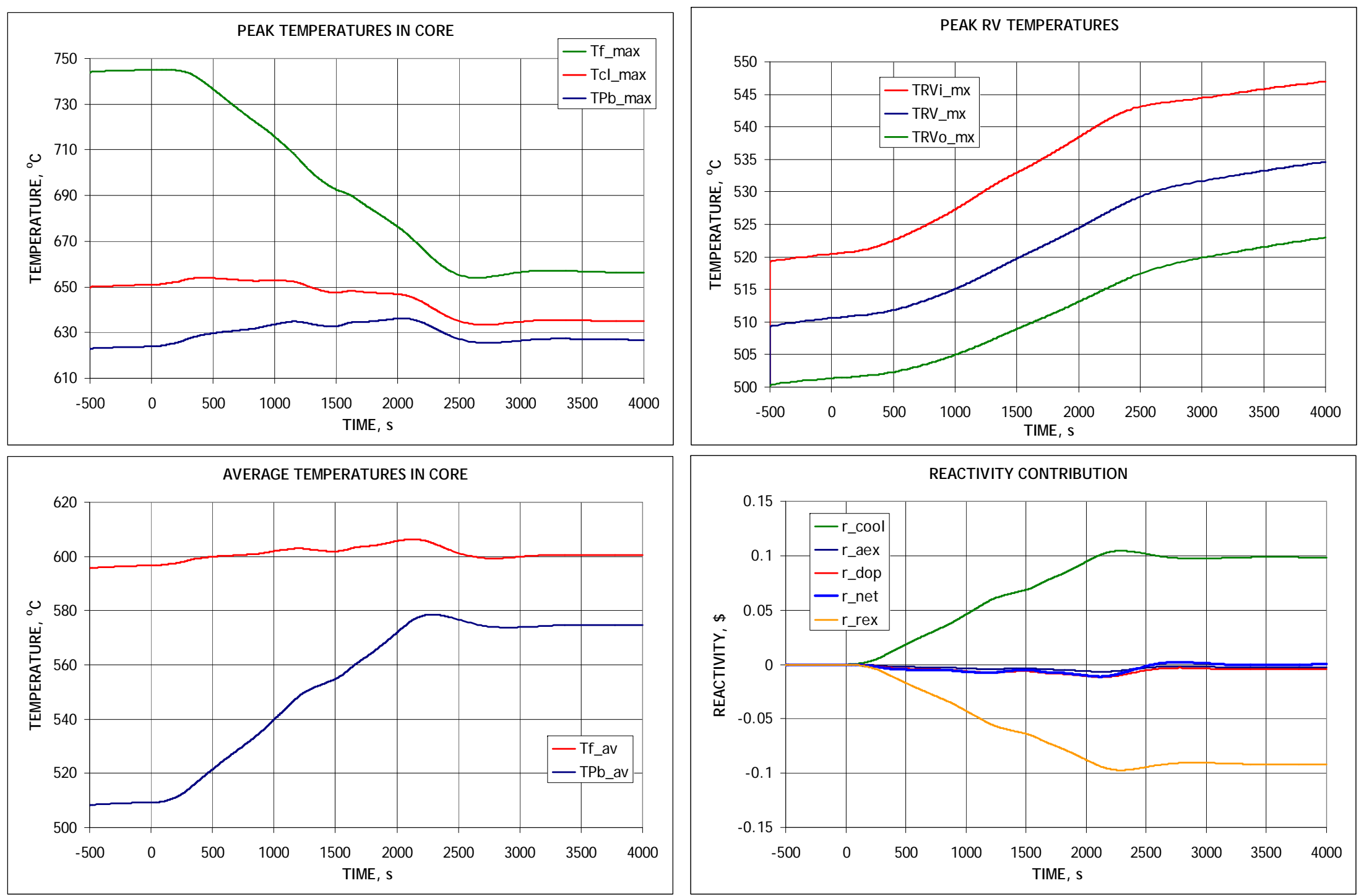

Figure 1-16. $100 \%$ Linear Decrease in Load (2). 

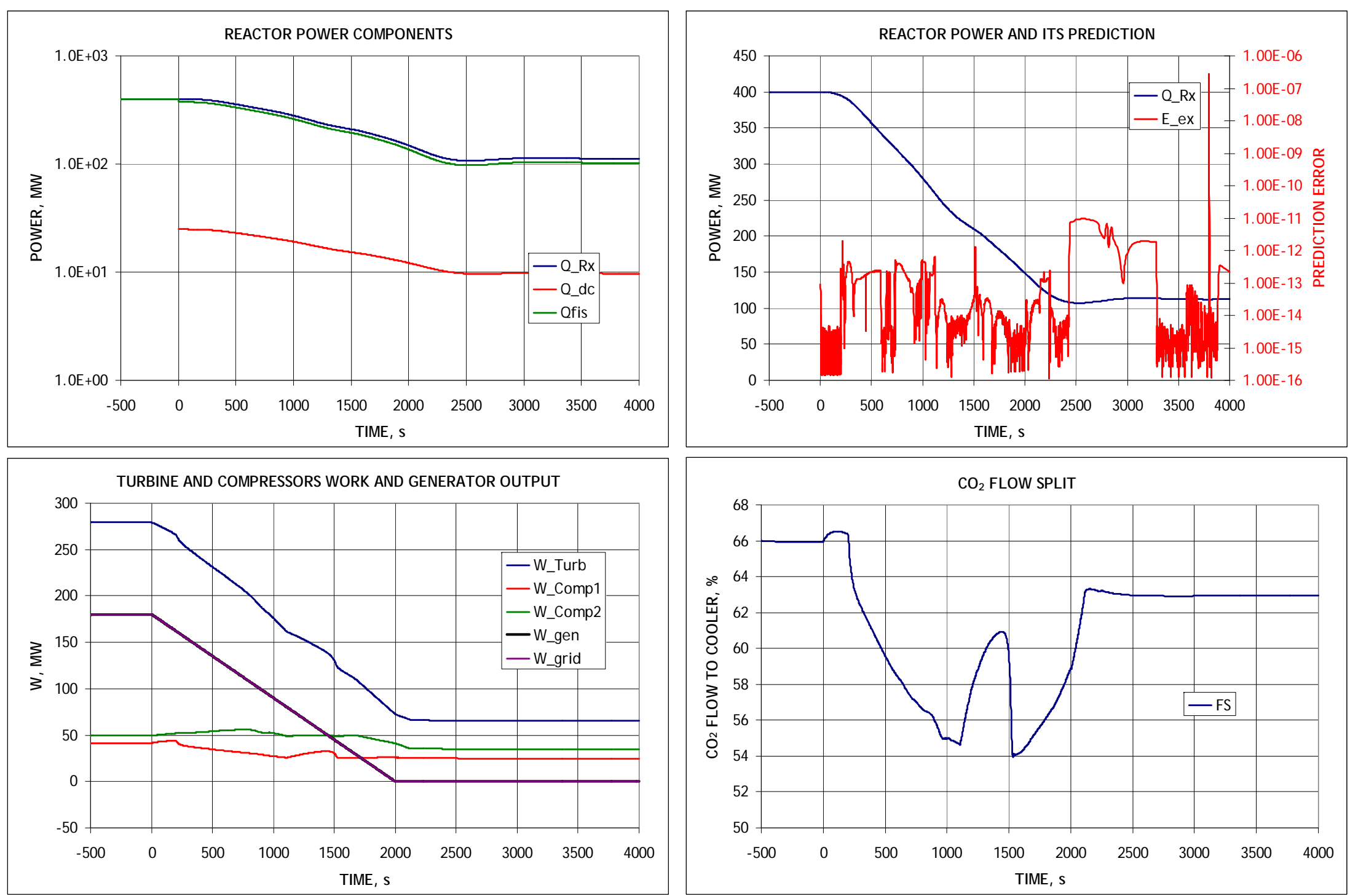

Figure 1-16. 100\% Linear Decrease in Load (3). 

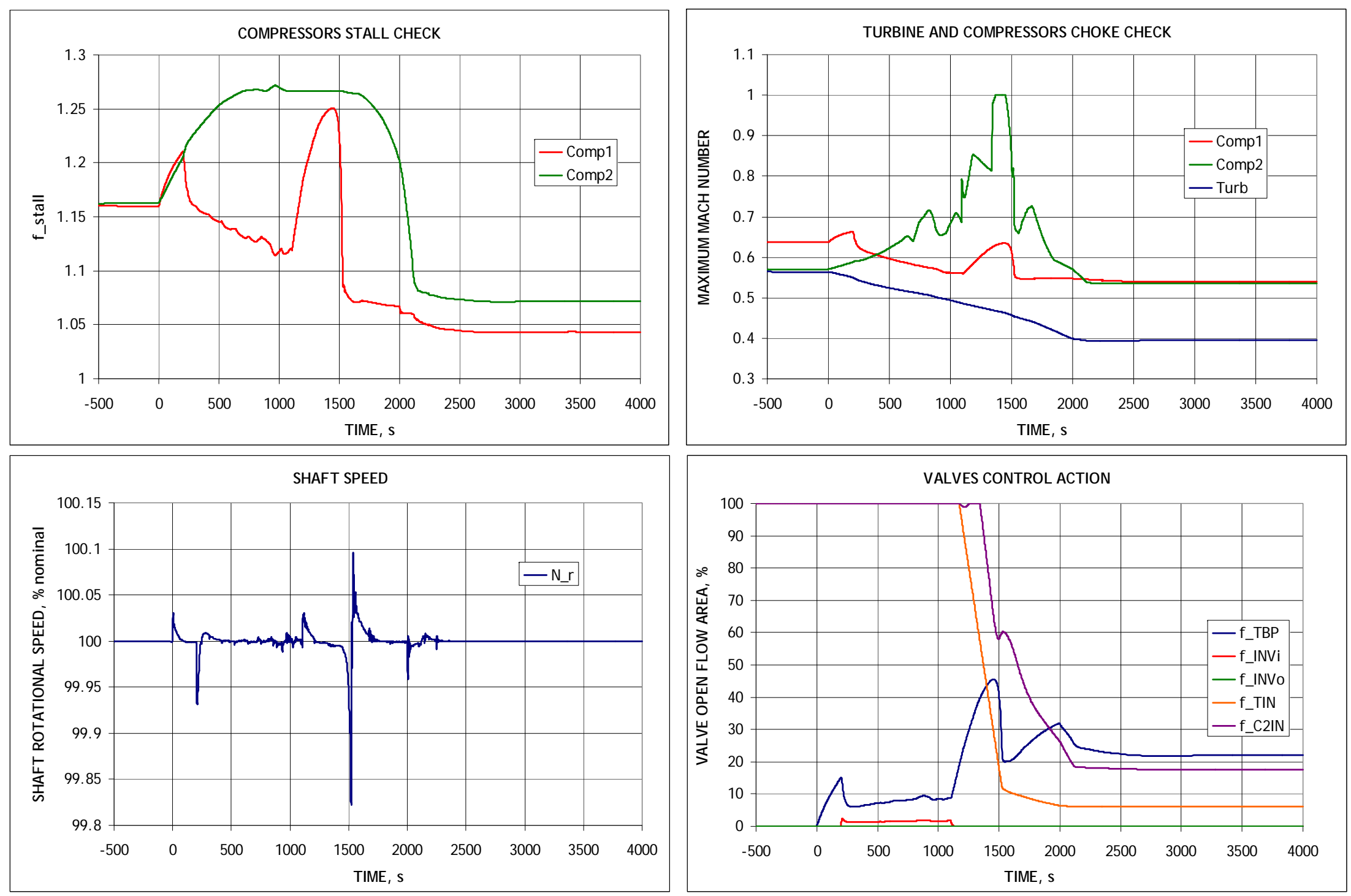

Figure 1-16. 100\% Linear Decrease in Load (4). 

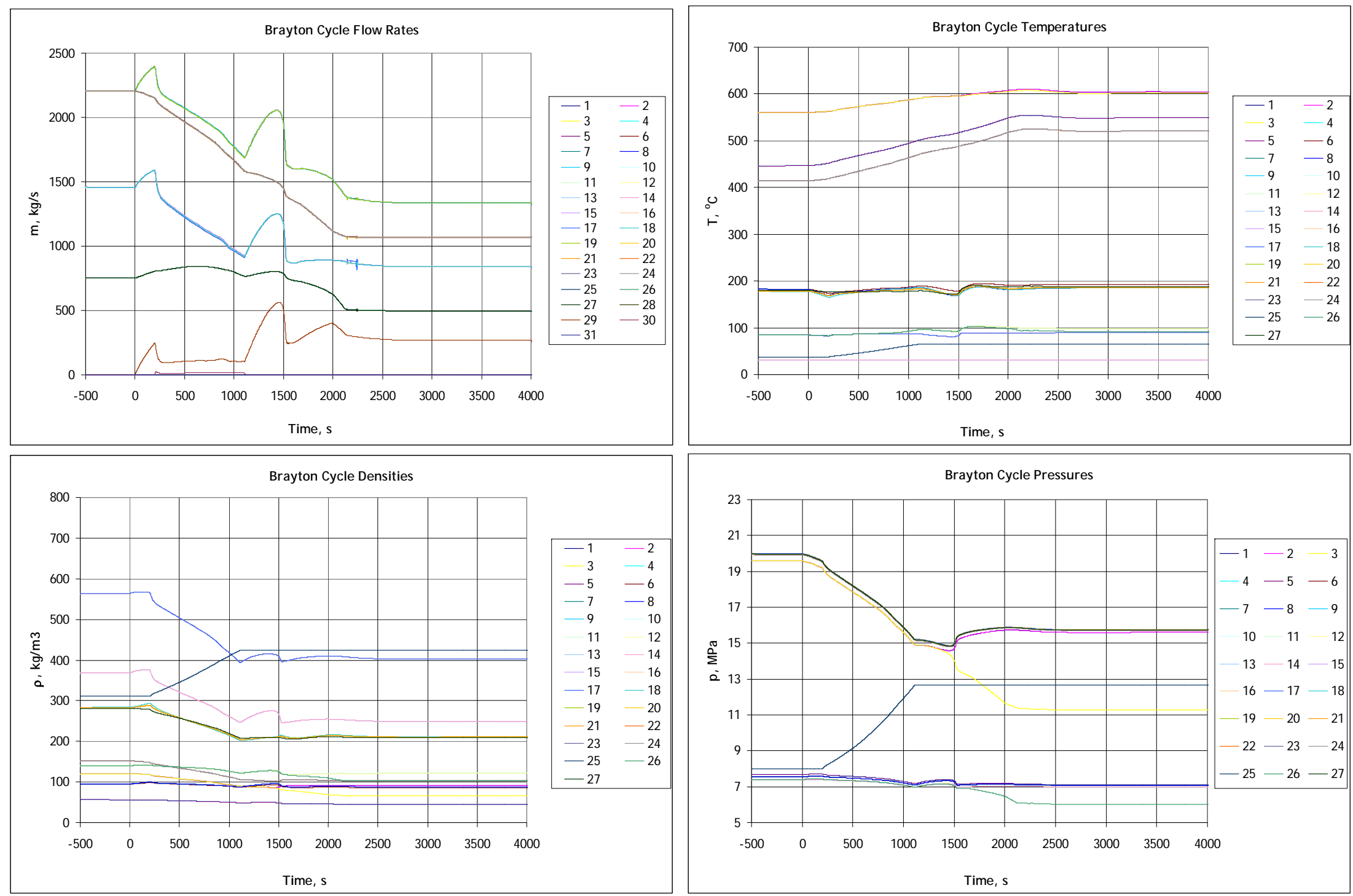

Figure 1-16. 100\% Linear Decrease in Load (5). 


\subsection{Summary and Proposed Future Developments}

\subsubsection{Dynamics Code}

\subsubsection{Code Development Summary}

A detailed system dynamics code for the supercritical carbon dioxide Brayton cycle has been developed. The code addresses the specific features of the system, such as non-ideal behavior of the working fluid, compressibility, and the recompression cycle configuration. The code is based on first-principles conservation equations. The code has been tested against steady-state conditions and demonstrated both stability and good agreement with steady-state calculations. The code has been applied to normal operational transients for the STAR-LM LFR. It is shown that the plant can be controlled from $0 \%$ to $100 \%$ power without exceeding limitations on either the $\mathrm{S}-\mathrm{CO}_{2}$ Brayton cycle or reactor conditions.

Since some assumptions are made during the implementation of the firstprinciples equations in the code, further improvements to the code could be made when any of these assumptions are revised or eliminated. Possible improvements to the code are discussed below.

\subsubsection{Turbomachinery Update}

The S- $\mathrm{CO}_{2}$ Brayton cycle turbomachinery design and performance models have been updated since the calculational results reported in this document were obtained. The improved turbomachinery models have to be incorporated into the code to generate new performance maps. Important for dynamics code improvement in the turbomachinery models has been incorporation of the turbine and compressor inlet nozzles into the model. This change leads to the fact that the minimum temperature in the cycle is now calculated between the inlet nozzle and the first stage of Compressor No. 1; i.e., inside the compressor. Since it is important to control this temperature to stay above the $\mathrm{CO}_{2}$ critical temperature, control of a temperature inside the compressor should be introduced to the code.

The other option to improve the turbomachinery treatment in the dynamics code would be introduction of dynamic equations describing flow inside the turbomachinery rather than the current instantaneous-response approach based on performance maps.

\subsubsection{PCHE Model}

Like the turbomachinery model, the model for Printed Circuit Heat Exchangers (PCHEs) has recently been updated. The model updates include better treatment of the geometry as well as heat transfer and pressure drop correlations. These updates would be 
very easily incorporated into the dynamics code since they do not involve revisions of the model and equations themselves.

\subsubsection{Cooler Model}

A major simplifying assumption of the current model is ideal control of the minimum cycle (i.e., cooler-outlet) temperature. This assumption allows usage of a simplified cooler model. A more realistic cooler model could be introduced into the code together with realistic temperature control. The major challenge here is that due to the significant properties variation, many axial nodes would be required to accurately calculate the heat transfer and pressure drop, significantly increasing total number of equations to be solved. Moreover, the $\mathrm{CO}_{2}$ compressibility near the critical point is expected to result in a decreased time scale for the momentum equations inside the cooler. Incorporating the accurate cooler model would require either improving the solution technique or modifying the code to run on multi-processor computers.

\subsubsection{Working Fluid Conditions}

The treatment of two-phase flow on the $\mathrm{CO}_{2}$ side should be added to the model if the simulation of the heat sink temperature variation would indicate that such a two-phase regime could be achieved in the cycle during particular transients or accidents.

\subsubsection{Normal Operational Transients}

\subsubsection{Summary of Normal Operation Analysis}

The analysis of the normal operational transients performed thus far has shown an acceptable system response during those transients - no major operational issues have been identified.

The dynamic calculations confirm the major conclusion from the steady-state load following analysis; that is., a combination of control mechanisms is required to control the $\mathrm{S}_{-} \mathrm{CO}_{2}$ Brayton cycle from $100 \%$ load to $0 \%$. Implementation of the dynamic code identified transient aspects of the cycle control, such as approaching compressor stall in the middle of some transients. These conditions will define the limits on speed of the load variation.

Further improvements in the plant control system logic could be made to eliminate minor issues, such as the transition from one control action to another. The results also showed that under some conditions, the Brayton cycle compressors could operate in an unstable (surge) regime. However, updated compressor designs (which have not yet been implemented in the dynamics code) have shown an increase in margin to stall/surge conditions which should improve the stability of the compressors and the whole cycle. 
The other possibility for control system modeling improvement would be incorporation of a more realistic control system model including the control instrumentation and electronics, and all of the delays associated with them.

\subsubsection{Startup and Shutdown, Decay Heat Removal}

The normal operating conditions should be expanded to include normal (nonemergency) plant start up and shutdown. Simulating these conditions, however, would require modeling of processes which are beyond of the current model capabilities, such as turbine start-up and the transition to the dedicated decay heat removal system. Therefore, significant model updates would be required.

\subsubsection{Heat Sink Conditions}

Incorporating the realistic cooler model would allow adding to the normal operating transients a set of conditions associated with the heat sink, such as the heat sink temperature and flow rate. It would also facilitate calculations to answer concerns about controllability of the $\mathrm{CO}_{2}$ conditions near critical point. (For code change requirements for the cooler model, see Section 1.5.1.4 above). 


\section{Centrifugal Compressor Models}

Industry review of earlier S- $\mathrm{CO}_{2}$ Brayton cycle development [11] indicated that centrifugal (radial-flow) compressors may have several advantages compared to axialflow compressors including wider operating range, better scalability, and better handling of two-phase flow in accident conditions. The disadvantage of radial turbomachinery has historically been lower efficiency. Since in the $\mathrm{S}-\mathrm{CO}_{2}$ Brayton cycle, the compressor work is significantly smaller than turbine work, the cycle efficiency is less sensitive to the compressor efficiency than to the turbine efficiency. Therefore, lower centrifugal compressor efficiency might not significantly penalize the cycle efficiency. On the other hand, operating range and two-phase flow are not concerns for the turbine, while its efficiency is very important for the cycle efficiency. For these reasons, it was decided to develop a centrifugal compressor model and investigate the effect of the compressor type on the system behavior. A centrifugal turbine model has also been developed, but it is not applied to the current $\mathrm{S}-\mathrm{CO}_{2}$ cycle design; however it may be used for comparisons with the results of small-scale tests incorporating a centrifugal turbine.

\subsection{Centrifugal Compressor Design and Performance Models}

The centrifugal compressor design and performance models are based on Reference [12]. A centrifugal compressor stage (Figure 2-1) consists of an inlet duct, inlet guiding vane (IGV), impeller, diffuser, and volute or collector. In case of a multistage compressor, the collector/volute is replaced with a crossover and a return channel for each stage, except for the last one. 


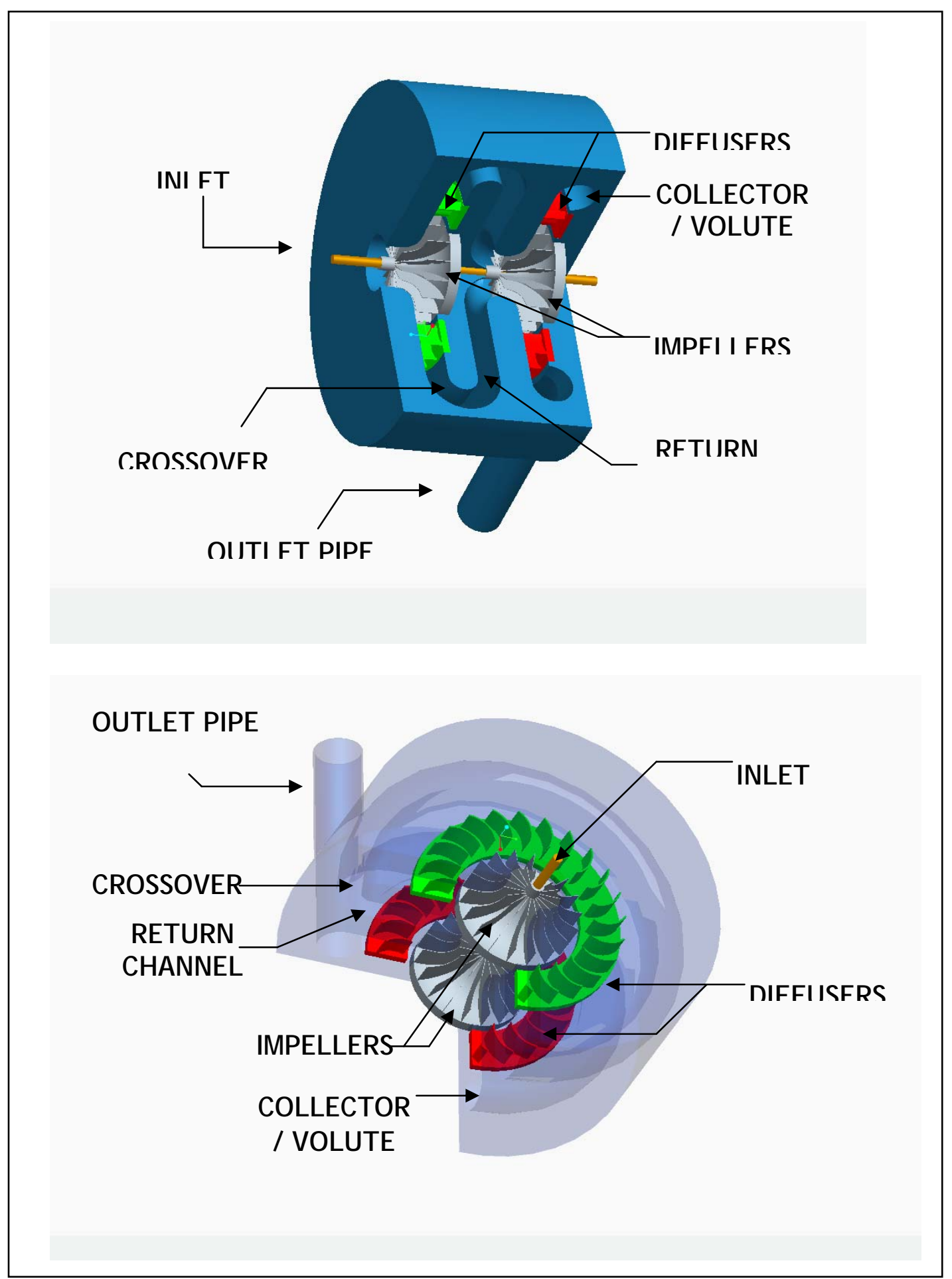

Figure 2-1. Illustration of a Two-Stage Centrifugal Compressor Design. 
The performance analysis of a centrifugal compressor is similar to that of an axial compressor. The main differences include:

- Different geometry, which is reflected by different loss correlations and different flow area calculations for the continuity equations, and a

- Change in blade speed due to the change in radius from impeller inlet to the impeller discharge, which affects the energy conservation equation as shown below.

Aside from these differences, the approach for a centrifugal compressor design and performance analysis is similar to that of an axial compressor. In particular, the same one-dimensional analysis is implemented under which flow conditions are calculated only at the impeller inlet and outlet (i.e., exact flow parameters inside an impeller are not calculated). Also, a realistic equation of state is used for $\mathrm{CO}_{2}$ properties; i.e., no ideal-gas simplifications are made.

The impeller performance is based on the energy conservation equation, equation of state, and loss correlation (Equations (2-1)-(2-4)).

Energy conservation equation:

$$
h_{t 2}^{\prime}=h_{t 1}^{\prime}+\left(U_{2}^{2}-U_{1}^{2}\right) / 2
$$

where

$$
h_{t}^{\prime}=h+\frac{W^{2}}{2}=\text { total relative enthalpy }
$$

Total outlet pressure for ideal process (without losses):

$$
p_{t 2 i d}^{\prime}=p\left(h_{t 2}^{\prime}, s_{1}\right)
$$

Total outlet pressure with losses:

where

$$
p_{t 2}^{\prime}=p_{t 2 i d}^{\prime}-f_{c}\left(p_{t 1}^{\prime}-p_{1}\right) \bar{\omega}
$$

$$
f_{c}=\frac{\rho_{t 2}^{\prime} T_{t 2}^{\prime}}{\rho_{t 1}^{\prime} T_{t 1}^{\prime}}=\text { correction factor to account for total pressure loss variation from }
$$

impeller inlet to impeller discharge,

$\bar{\omega}=$ loss coefficient.

The loss coefficient is calculated as a sum of loss components (such as, incident, diffusion, choke, and so on), as described in Reference [12],

$$
\bar{\omega}=\bar{\omega}_{i n c}+\bar{\omega}_{D I F}+\bar{\omega}_{C H}+\bar{\omega}_{S F}+\bar{\omega}_{B L}+\bar{\omega}_{H S}+\bar{\omega}_{\lambda}+\bar{\omega}_{m i x}+\bar{\omega}_{C L}+\bar{\omega}_{c r}
$$

The impeller design is based on so-called "good design practice" [12]. Under this approach, the design parameters (such as impeller blade angle at discharge, for example) 
are expressed as empirical functions of flow coefficient. The flow coefficient is based on the volumetric inlet flow rate, impeller discharge radius, and rotational speed:

$$
\phi=\frac{\dot{m}}{\rho_{0} \pi r_{2}^{2} U_{2}}
$$

The parameters selected based on "good design practice" include:

- Blade load coefficient, which defines the change in the tangential component of flow speed,

$$
\begin{aligned}
& I_{B}=C_{U 2} / U_{2}-U_{1} C_{U 1} / U_{2}^{2} \\
& I_{B}=0.68-(\phi / 0.37)^{2}
\end{aligned}
$$

- The slip factor, which defines the blade angle at discharge

$$
\begin{aligned}
& \sigma=1-\sqrt{\cos \kappa_{2}} \sin \alpha_{C 2} / z^{0.7} \\
& I_{B}=\sigma\left(1-\lambda \phi_{2} \tan \kappa_{2}\right)-U_{1} C_{U 1} / U_{2}^{2}
\end{aligned}
$$

- Blade, flow, and incidence angles at impeller inlet,

$$
\begin{aligned}
& \beta_{1}=-60^{\circ} \\
& \alpha_{1}=0^{\circ} \\
& i_{1}=0^{\circ} \\
& i_{1}=k_{1}-\beta_{1}
\end{aligned}
$$

- Flow angle at discharge, and

$$
\cot \alpha_{2}=0.26+3 \phi
$$

- Impeller axial length,

$$
\Delta z_{I}=\left(0.014+0.023 d_{2} / d_{h}+1.58 \phi\right) d_{2}
$$

All other design parameters are calculated from continuity and Euler equations and from velocity triangles. The impeller discharge radius, $r_{2}$, which goes into the definition of the flow coefficient, is selected such that the stage-discharge pressure matches the design value.

The limiting design criterion is a blade loading which is designed as a ratio of an average blade velocity difference to the average velocity in the impeller. The design value for the blade loading should not exceed 0.9:

$$
\frac{2 \Delta W}{W_{1}+W_{2}} \leq 0.9
$$

where

$$
\Delta W=2 \pi d_{2} U_{2} I_{B} /\left(z L_{B}\right)=\text { average blade velocity difference. }
$$


The blade loading limitation is used to calculate the required number of impeller blades, $z$, according to Equation (2-6).

The design and performance of a diffuser is similar to that of the impeller, except that there is no rotation and there is no external energy input in the diffuser. Again, some parameters are calculated based on conservation equations; the rest is assumed based on "good design practice" to match the impeller design. Both design parameters and the loss coefficient depend on whether a vaned or vaneless diffuser is utilized (both are supported by the code). The loss coefficient for a vaned diffuser has several components, defined in Reference [12].

The fluid conditions at the diffuser outlet are defined by the conservation of total energy and the change in total pressure due to losses (Equations (2-7), (2-8)). The continuity equation relates the outlet density to the flow speed used in the energy equation.

$$
h_{t 3}=h_{t 4}
$$

where

$$
\begin{aligned}
& h_{t}=h+\frac{C^{2}}{2}=\text { total enthalpy. } \\
& p_{t 4}=p_{t 3}-\left(p_{t 3}-p_{3}\right) \omega
\end{aligned}
$$

The volute or collector is not currently modeled in the centrifugal compressor design and analysis codes. That is, it is assumed that the flow conditions at the compressor outlet are the same as the conditions at the diffuser discharge. The return channel is modeled with an assumed value of the pressure loss coefficient. 


\subsection{Implementation of the Centrifugal Compressor Models}

The centrifugal compressor models that have been developed have been applied to the $\mathrm{S}-\mathrm{CO}_{2}$ Brayton cycle. For this investigation, the $\mathrm{S}-\mathrm{CO}_{2}$ Brayton cycle was designed for the application currently pursued under the Generation IV Nuclear Energy Systems Initiative - a 20 MWe power converter for the Small Secure Transportable Autonomous Reactor (SSTAR) LFR (compared to 180 MWe STAR-LM reported in Section 0). The models have been applied to the steady-state design code, as well as steady-state load following code. Incorporation of the centrifugal compressor models into the Plant Dynamic Code is currently in progress.

\subsubsection{Steady-State Cycle Analysis}

The centrifugal compressor design model has been implemented into the $\mathrm{S}-\mathrm{CO}_{2}$ design code used to calculate the efficiency of the centrifugal $\mathrm{S}-\mathrm{CO}_{2}$ compressors in comparison with axial compressors and to investigate the effects of compressor design on cycle performance. It should be noticed here that the centrifugal compressor designs were not optimized for this application yet. ${ }^{1}$ For example, the effect of the number of stages on the compressor and cycle efficiencies has not been carried determined. Rather the simplest design which provides a reasonable efficiency has been adopted for the analysis and for comparison with the axial design.

Table 2-1 shows the main design parameters and efficiencies of the axial and centrifugal compressors. The turbine data is also presented for comparison.

Table 2-1. Comparison of Axial and Centrifugal Compressors

\begin{tabular}{|l|c|c|c|c|c|}
\hline & Turbine & \multicolumn{2}{|c|}{ Compressor No. 1 } & \multicolumn{2}{c|}{ Compressor No. 2 } \\
\hline Type & Axial & Axial & Centr. & Axial & Centr. \\
\hline Number of stages & 5 & 8 & 1 & 9 & 2 \\
\hline Efficiency, TS, \% & 92.0 & 88.0 & 88.1 & 86.8 & 88.7 \\
\hline Max. diameter $^{2}, \mathrm{~m}$ & 0.34 & 0.21 & 0.59 & 0.28 & 0.60 \\
\hline Length $^{3}$ (stages), m & 0.48 & 0.22 & 0.10 & 0.18 & 0.18 \\
\hline Length (with diffuser), m & 1.80 & 1.68 & $\mathrm{n} / \mathrm{a}$ & 1.82 & $\mathrm{n} / \mathrm{a}$ \\
\hline Min. hub diameter, cm & 19.4 & 17.4 & 9.8 & 26.2 & 10.6 \\
\hline Min. blade height, cm & 3.4 & 1.1 & 0.7 & 0.5 & 0.6 \\
\hline Max. blade height, cm & 7.5 & 1.7 & 2.8 & 1.1 & 3.1 \\
\hline & & & & & \\
\hline Cycle efficiency & & & & \\
\hline
\end{tabular}

\footnotetext{
${ }^{1}$ The detailed centrifugal compressor design optimization for the $\mathrm{S}-\mathrm{CO}_{2}$ Brayton cycle has been carried out for asodium-cooled fast reactor under a different project.

${ }^{2}$ Without casing and volute/collector.

${ }^{3}$ Estimated.

${ }^{4}$ Cycle efficiency is shown for the two compressors having similar designs (either both axial or both centrifugal).
} 
It follows from Table 2-1 that centrifugal compressors achieve the same or even slightly better performance with significantly fewer stages. The only disadvantage of the centrifugal compressor designs is the relatively large radial dimensions (which could complicate mounting them on the same shaft with the turbine and generator). The resulting cycle efficiencies are very close for the axial and centrifugal designs.

\subsubsection{Off-Design Performance Analysis}

The performance of the centrifugal and axial compressors at off-design conditions was compared using the steady-state load following code. The investigation of the cycle behavior under various control actions was carried out in the same way as reported in [1]. Even though the systems investigated in [1] and here are very similar (both cycles are designed for LFRs with similar temperatures and efficiencies), the power levels of the LFR plants are different resulting in different designs for the compressors and turbines. Therefore, the results could not be compared directly, but major trends in the behavior of the different systems should be the same.

\subsubsection{Compressor Performance Maps}

Figure 2-2 compares the performance maps (pressure ratio and efficiency versus flow rate) for the design inlet conditions. Figure 2-2 confirms the expected increased operating range of the centrifugal compressors, which is expected to improve the operating range of the cycle control mechanisms. Also, the efficiency curve is more flat for the centrifugal compressors meaning that good performance should be maintained at wider flow rate variations.

However, Figure 2-2 also demonstrates that centrifugal compressors could not achieve same pressure ratios as axial compressors could. This may affect the cycle performance under conditions where the compressor pressure ratio tends to increase (such as during turbine throttle control). 

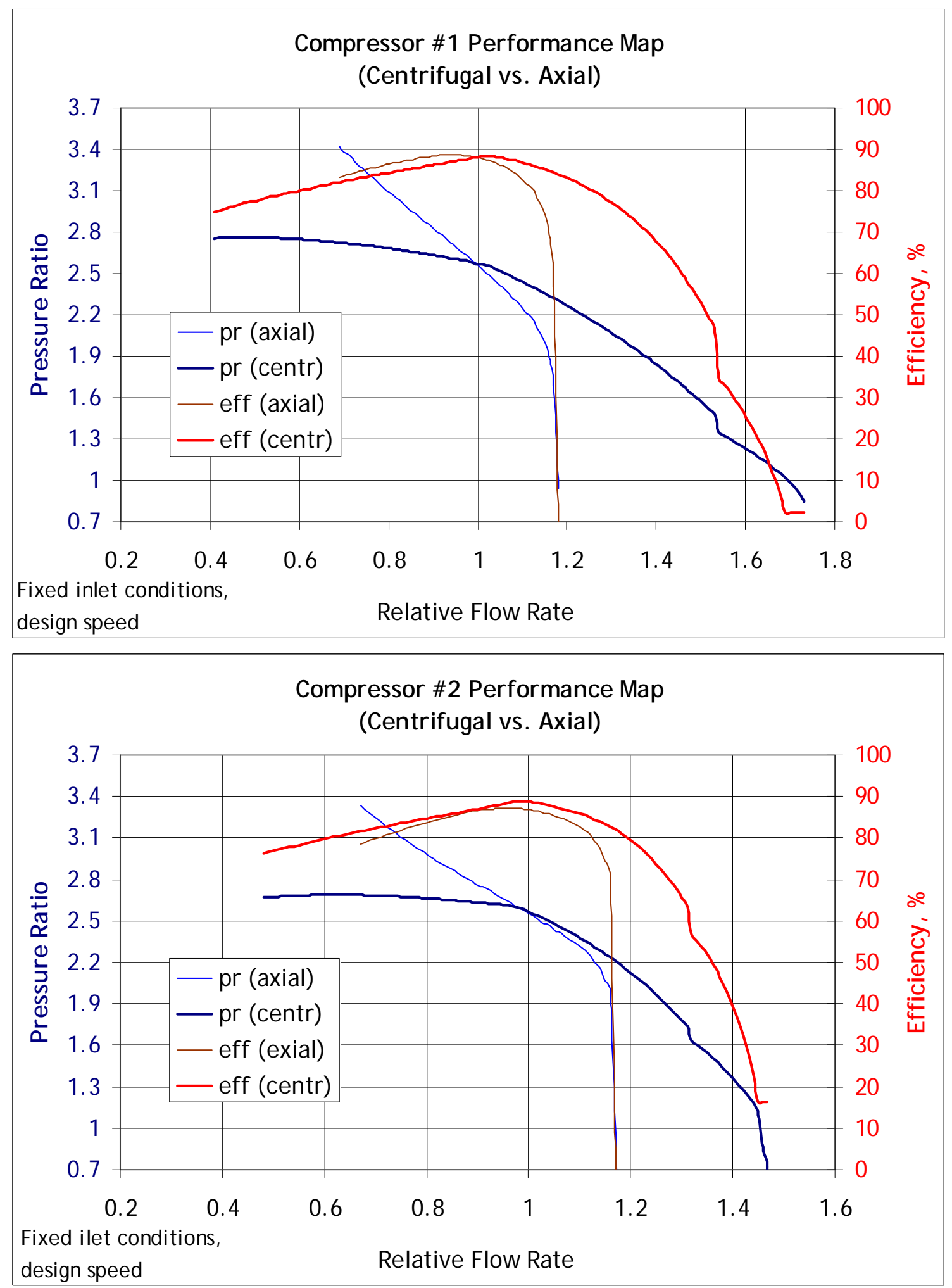

Figure 2-2. Comparison of the Performance Maps for Axial and Centrifugal S-CO Compressors. 


\subsubsection{Turbine Bypass Control}

In this control scheme, part of the flow is bypassing HTR, IRHX, and turbine. As a result, the turbine work is reduced as is the generator load. Calculations of the system response with centrifugal compressors (Figure 2-3) show that this control mechanism is capable of controlling the system from $100 \%$ all the way down to $0 \%$ load. However, since the compressor pressure ratios are decreasing under this control approach, according to Figure 2-2, the flow rate through the centrifugal compressors is higher than what it would be for axial compressors (for the same pressure ratio). As a result, the flow rate through the turbine (and, therefore, the IRHXs) is decreasing less in case of centrifugal compressors (compared to axial compressors). As a result, the autonomous reactor control does not change the reactor power significantly. Therefore, the excess of the heat produced in the reactor is going to the cooler such that the heat removal requirement in the cooler is increasing demanding a significant increase in the water flow rate and water pumping power. Figure 2-3 shows a significant increase in the cooling water pumping power, which might not be feasible unless a separate high-power pump is reserved in the system. (Pumping power for the STAR-LM LFR system with axial compressors remained approximately flat over the whole range of control action).

As a result, although centrifugal compressors have a wider operating range, their flatter pressure ratio curves result in the need for a greater cooler water side pumping power.

\subsubsection{Inventory Control}

Under inventory control, some mass of $\mathrm{CO}_{2}$ is removed from the cycle. As a result, the system pressures and flow rate are decreasing reducing the turbine work and generator output. In a system with centrifugal compressors (Figure 2-4), a load reduction down to about $20 \%$ nominal can be achieved without reaching the limits on compressor operating range.

However, the real limitation in this control scheme is the pressure inside of the inventory tank. As $\mathrm{CO}_{2}$ inventory is removed from the $\mathrm{S}-\mathrm{CO}_{2}$ cycle into the inventory tank, the system pressures decrease while the tank pressure increases. At the same time, in order for the control to operate (without additional compressors), the tank pressure should remain below the high system pressure. Figure 2-5 shows the increase in tank pressure due to mass accumulation for various tank volumes (the HTR total volume is given for comparison). Inventory control with a tank(s) of total volume about the same as that of HTR is capable of controlling the system from about $30 \%$ up to $100 \%$ load. The previous lower limit for the STAR-LM system with axial compressors was $50 \%$ load. 

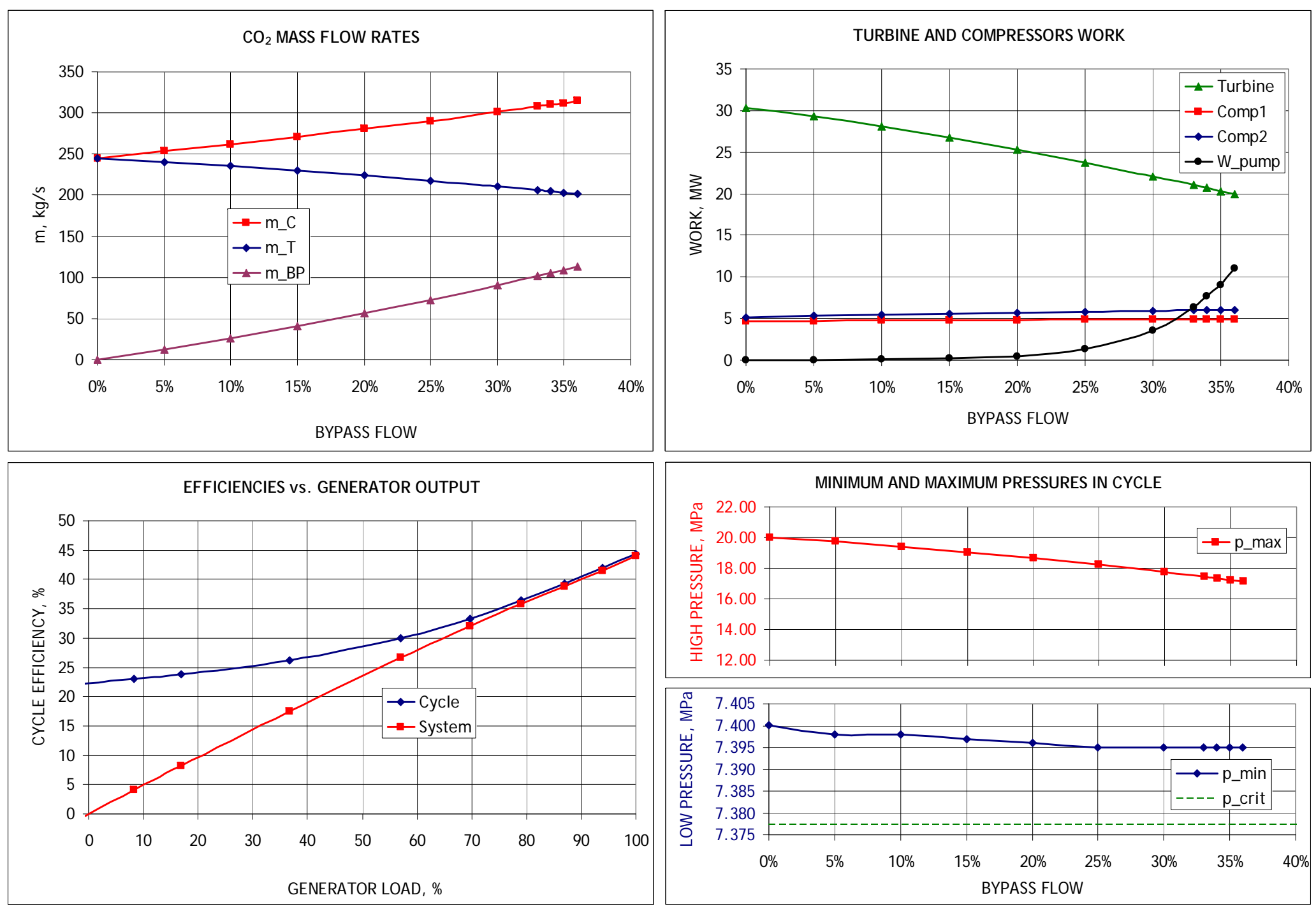

Figure 2-3. Turbine Bypass Control with Centrifugal Compressors. 

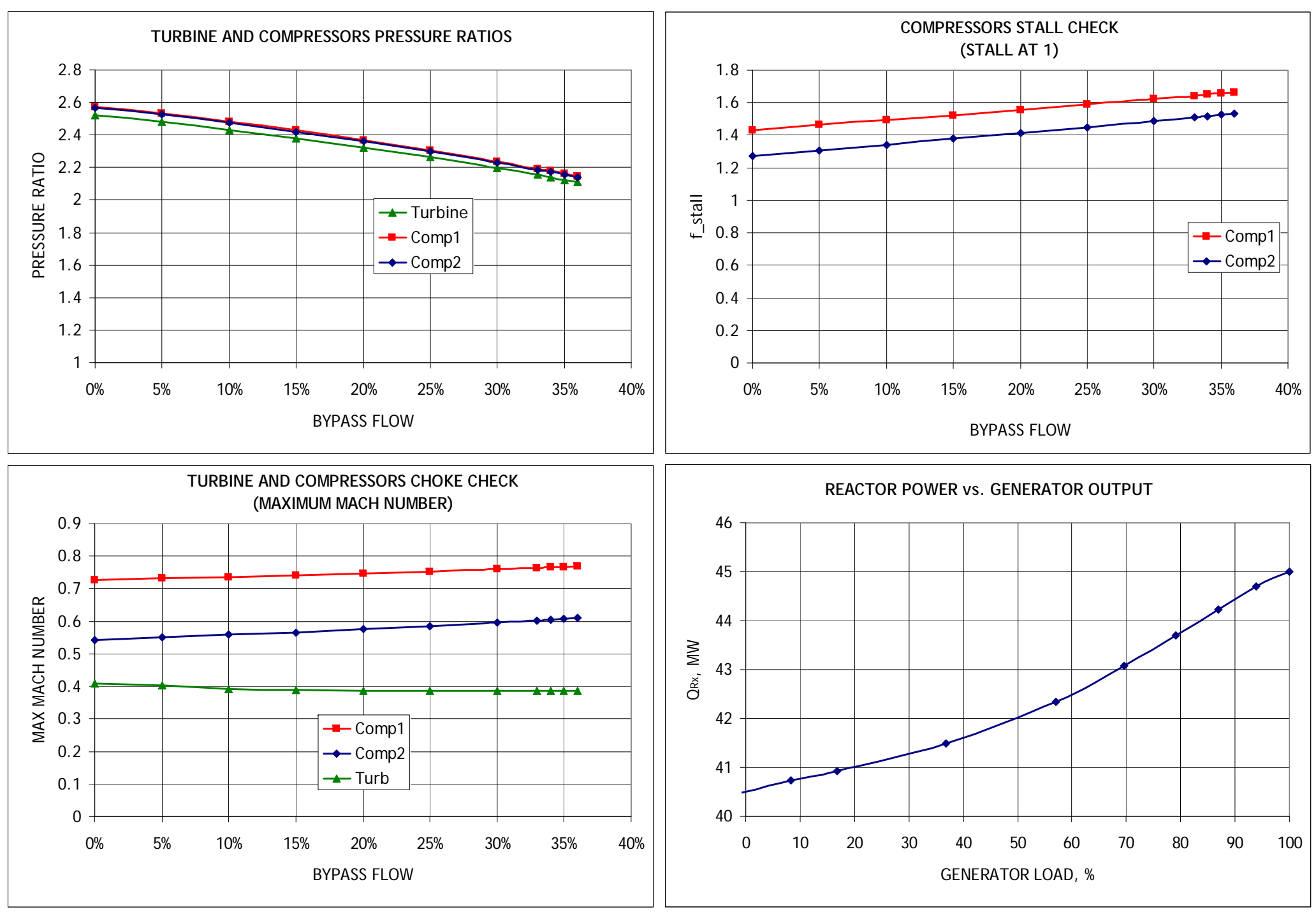

Figure 2-3 (Continued). Turbine Bypass Control with Centrifugal Compressors. 

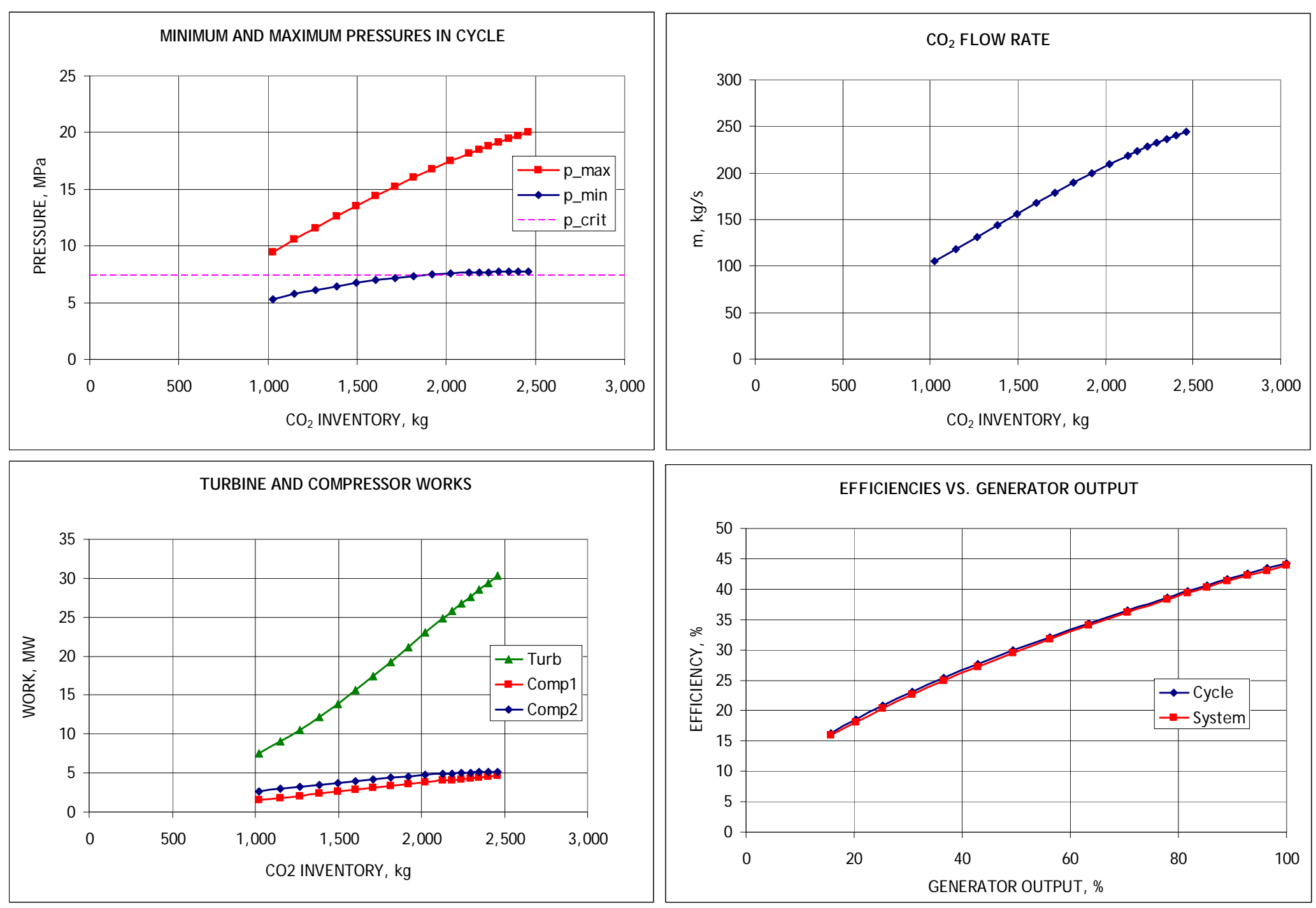

Figure 2-4. Inventory Control with Centrifugal Compressors. 

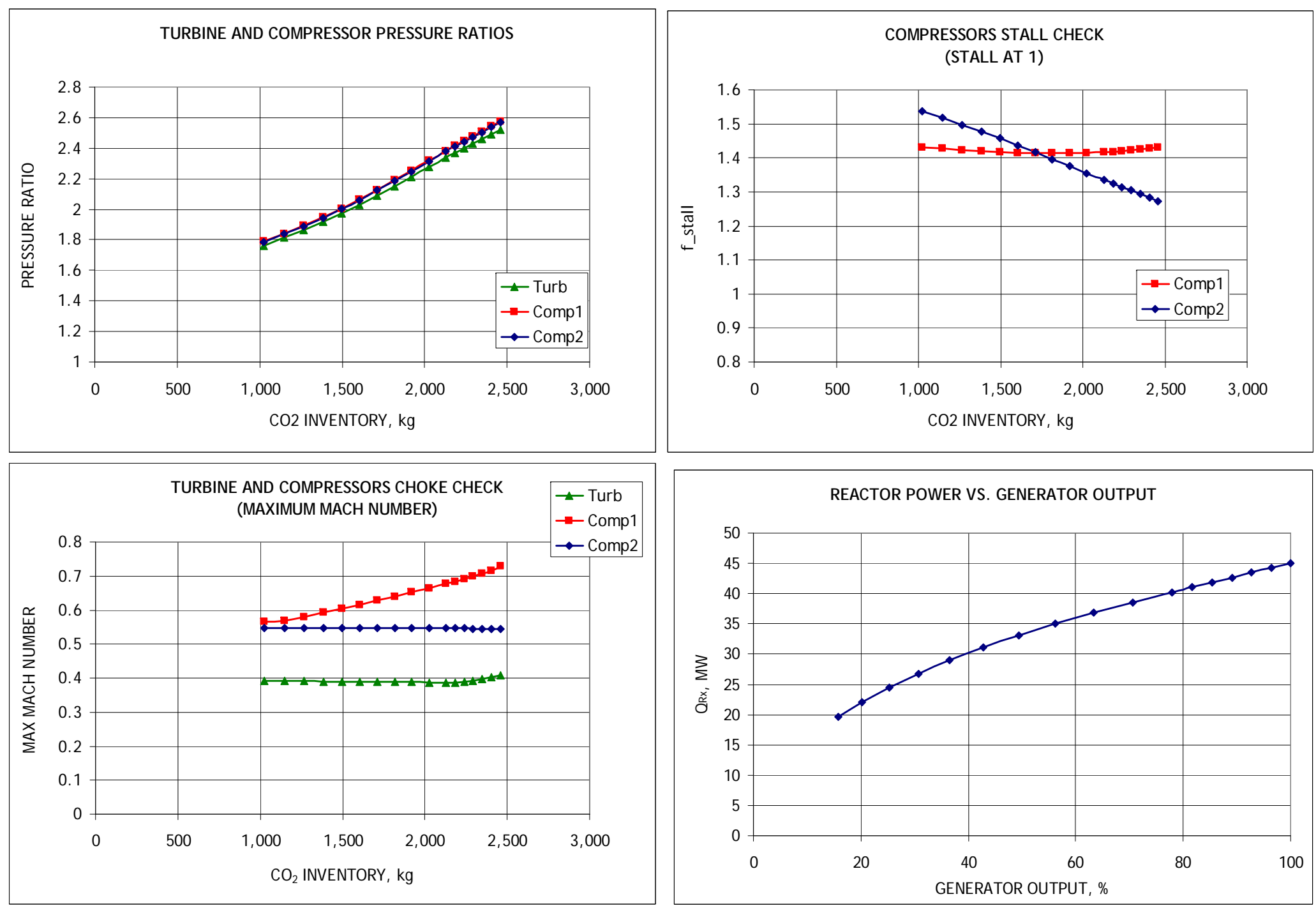

Figure 2-4 (Continued). Inventory Control with Centrifugal Compressors. 

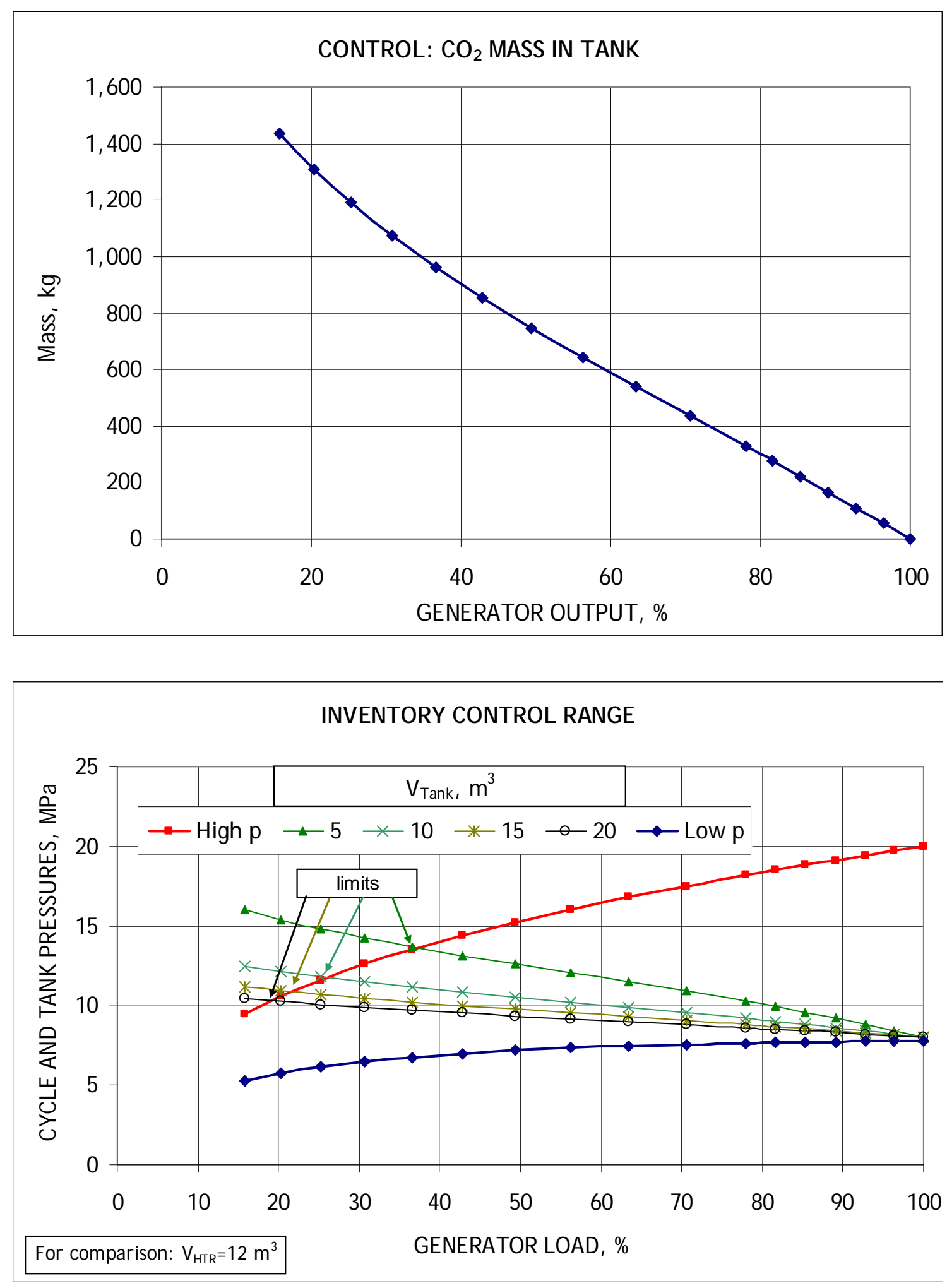

Figure 2-5. Inventory Accumulation in Tank and Tank Pressure. 


\subsubsection{Turbine Inlet Valve Control}

Under this control mechanism, the pressure ratio in the turbine is reduced by introducing a pressure drop before the turbine by means of a throttle valve. As a result, the turbine pressure ratio decreases, while the compressor pressure ratio increases (Figure 2-6). Since centrifugal compressors can achieve a significantly lower pressure ratio before stall compared to axial designs (Figure 2-2), compressor stall is predicted earlier at approximately 50\% load, according to Figure 2-6. The STAR-LM system with axial compressors reached about $35 \%$ load before the onset of a compressor stall.

\subsubsection{Summary of Load Following Analysis for Centrifugal Compressors}

The increased operating range of the centrifugal compressors helps widen the effective range of the control mechanisms in terms of staying away from compressor stall or choking conditions. However, the flatter pressure ratio curves for centrifugal compressors introduced new limitations on the control ranges. Overall, the ranges of control mechanisms for the $\mathrm{S}-\mathrm{CO}_{2}$ Brayton cycle with centrifugal compressors are close to those calculated previously for the cycle with axial compressors. In particular, a combination of control mechanism is still required to effectively control the $\mathrm{S}-\mathrm{CO}_{2}$ Brayton cycle with centrifugal compressors over the entire operating range.

Given that the steady-state and load-following performance of the $\mathrm{S}-\mathrm{CO}_{2}$ Brayton cycle with centrifugal compressors is close to that with axial compressors, no preferences could be made on the compressor design selection at this point. The selection should be made based on cost analysis and whether or not two-phase flow is calculated to be possible at the compressor inlets during transient simulation. Since both these factors are expected to benefit centrifugal compressors (for example, the fewer stages for centrifugal compressors should reduce the cost), the centrifugal compressor design will remain the reference choice for now. 

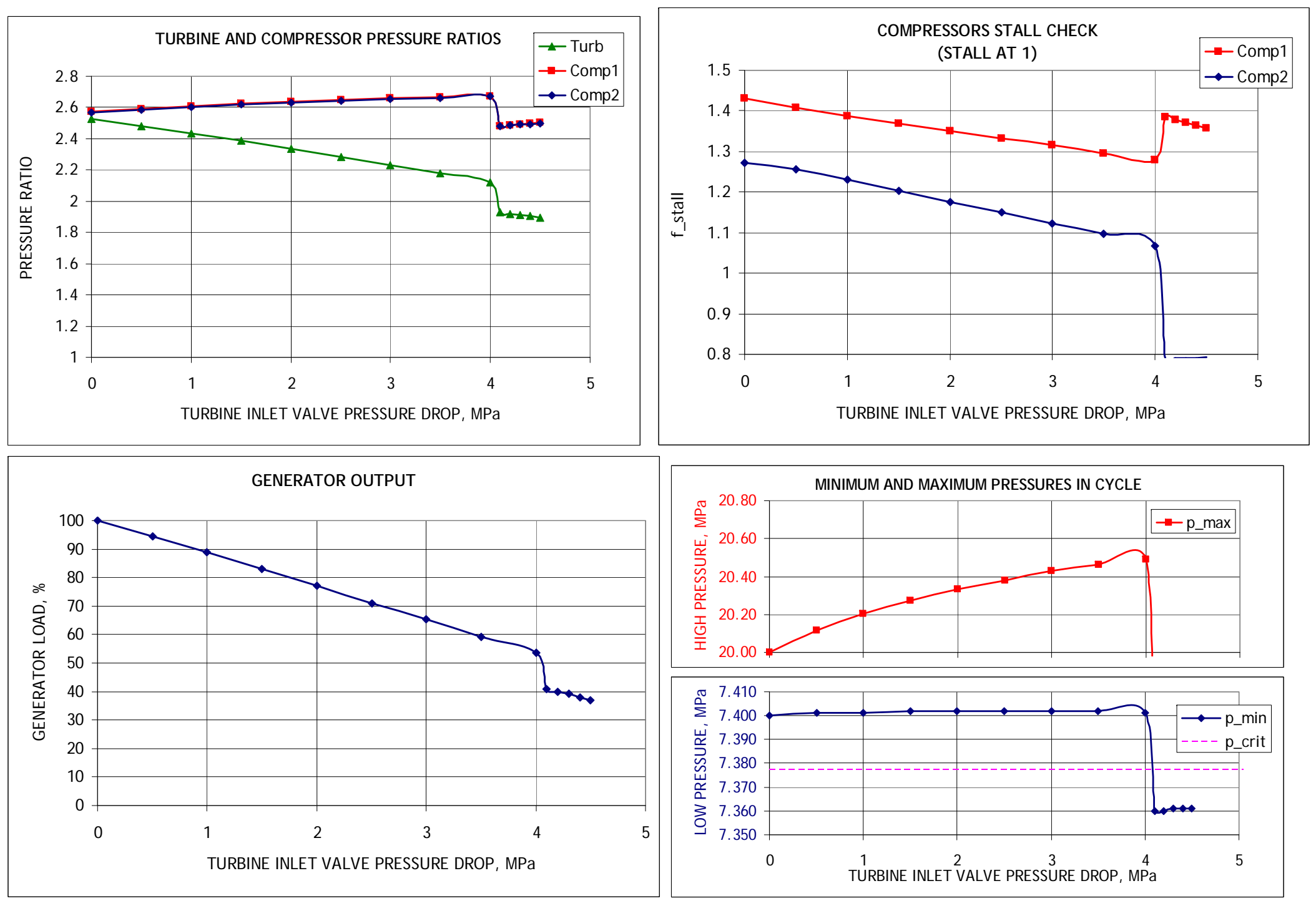

Figure 2-6. Turbine Inlet Valve Control with Centrifugal Compressors. 


\section{Radial Inflow Turbine Analysis}

\subsection{Introduction}

Argonne National Laboratory is participating in a comparison of models and codes with data obtained with the Sandia Brayton Loop located at Sandia National Laboratories (SNL) in Albuquerque. SNL has procured a closed Brayton loop operating with gases or mixtures of gases as the working fluid at atmospheric pressure and nominally at 30 degrees Celsius temperature at the compressor inlet. The Sandia Brayton Loop (SBL-30) incorporates a compressor, turbine, recuperator, and $63 \mathrm{KW}$ electrical heat source, and operates at temperatures up to 630 degrees Celsius. The loop is fully instrumented with thermocouples, pressure transducers, a flow meter, shaft speed, and power generation meters. The loop is capable of being operated with gases or gas mixtures but not with supercritical $\mathrm{CO}_{2}$. Tests have been conducted to generate data useful for the early testing and partial validation of turbomachinery models and dynamics codes such as characteristic flow curves, static Brayton cycle tests, dynamic Brayton cycle tests, and control simulation tests. The SBL-30 loop incorporates a radial inflow turbine as well as a radial flow compressor. In contrast, the use of axial flow turbines has been envisioned for the commercial-scale $\mathrm{S}-\mathrm{CO}_{2}$ Brayton cycle due to the higher efficiencies possible with axial flow turbines combined with their operation well away from the critical point.

Although radial turbines are not used in current $\mathrm{S}-\mathrm{CO}_{2}$ Brayton cycle energy converter preconceptual designs, many of the equations are similar for axial and radial turbines. For this reason, validation of a radial turbine model against experiment data is of interest for axial turbine models. In addition, a radial turbine may be the design-ofchoice recommended by a commercial vendor for a small-scale demonstration of the S$\mathrm{CO}_{2}$ Brayton cycle. ANL has developed a computer code to model the behavior of a radial turbine based upon use of a commercially available Capstone C-30 micro turbine. This section of the report is a description of the techniques applied to the analysis performed on the radial inflow turbine using an aerodynamic performance analysis discussed by Aungier [13].

\subsection{Theory and Analysis}

Aerodynamic performance analysis is an essential aspect of the aerodynamic design for the radial inflow turbine and application. The most effective approach to doing a performance analysis is using a one-dimensional method sometimes called the meanline method approach. The one-dimensional method relies on the analysis of the flow along a mean stream surface through various stage components. However, it is important to note that the best a one-dimensional analysis can do is predict the expected performance assuming that the geometry is based on good design practice. In comparison to other types of turbo-machinery, such as the radial compressor design where years of practice have fine tuned the performance analysis codes, the aerodynamic performance 
analysis for the radial in-flow turbine is relatively immature. For this reason, the analysis approach shall be described in detail.

\subsubsection{Boundary Layer Loss Coefficient}

The profile loss coefficient, $Y_{p}$, is expressed in terms of the ideal (no loss) component discharge dynamic head rather than the inlet dynamic head due to surface boundary layers in compressor cascades. The derivation of this approximation for the total pressure losses assumes the instant mixing of the boundary layers and mainstream fluid while conserving mass and momentum. This profile loss coefficient works for nozzles and rotors as they both have end-wall and blade surface boundaries, which overlap in the passage corners.

$$
Y_{p}=\frac{\Delta P_{t}}{P_{t 3, i d}-P_{3}}=\frac{2 \Theta+\Delta^{2}}{(1-\Delta)^{2}}+\sum \Delta Y
$$

where,

$$
\begin{aligned}
& \Theta=\sum \theta / b \\
& \Delta=\sum \delta^{*} / b
\end{aligned}
$$

The normalized defect thicknesses used in the loss coefficient equation are better defined by,

$$
\begin{aligned}
& \Theta=1-\left[1-\sum \theta_{w} / b_{w}\right]\left[1-\sum \theta_{b} / b_{b}\right] \\
& \left.\Delta=1-\left\lfloor 1-\sum \delta_{w}^{*} / b_{w}\right\rfloor 1-\sum \delta_{b}^{*} / b_{b}\right\rfloor
\end{aligned}
$$

where the effective blade-to-blade pitch passage is calculated from the blade pitch and the camber line angle at the blade discharge,

$$
b_{b}=s_{3} \sin \beta_{3}
$$

The boundary layer momentum thickness at the component discharge is,

$$
\theta=c_{f} \rho_{\text {ave }}\left[\left(u_{1} / u_{3}\right)^{5}+2\left(u_{2} / u_{3}\right)^{5}+1\right] L /\left(8 \rho_{3}\right)
$$

where,

$$
\begin{aligned}
& u_{1}=\text { inlet velocity } \\
& u_{2}=\text { throat velocity } \\
& u_{3}=\text { discharge velocity } \\
& L=\text { flow path length } \\
& c_{f}=\text { friction coefficient }
\end{aligned}
$$

Here the average density takes into account the inlet, throat, and exit density as, 


$$
\rho_{\text {ave }}=\left[\rho_{1}+2 \rho_{2}+\rho_{3}\right] / 4
$$

Also the coefficient of friction, $c_{f}$, is defined here as it is used in the compressor. The displacement thickness by definition of the shape factor is given by,

$$
\delta^{*}=H \theta
$$

The boundary layer thickness, $\delta$, is defined by the power-law velocity profiles. It is assumed that the boundary layer shape factor for the $1 / 7^{\text {th }}$ power-power law velocity profile is $H$.

where

$$
\delta=\theta H(H+1) /(H-1)
$$

$$
H=1.2857
$$

\subsubsection{Volute Analysis}

The passage area is defined by,

$$
A=2 \pi r b
$$

The first component for analysis is the volute. The thermodynamic conditions are determined from the inlet temperature, pressure, and inlet flow rate. For this stationary component, neglecting frictional losses, the total enthalpy is conserved, thus it is found that $H_{1}=H_{3}$. Station 1 is defined as the inlet and Station 3 as the outlet of the component in consideration throughout this report. Hence, Station 2 is a point between the inlet and the outlet of the component. The total static enthalpy of the volute is defined by,

$$
h_{3}=H_{3}-\frac{1}{2} C_{3}^{2}
$$

\subsubsection{Nozzle Analysis}

The nozzle row in the radial inflow turbine consists of a simple radial passage of constant width. Equation (3-13) is used to define the flow passage area. With the data found from the outlet of the volute, one can once again find the new thermodynamic conditions of the fluid.

$$
A=b\left[2 \pi r \sin \beta-t_{b} N\right]
$$

where,

$$
\begin{aligned}
& b=\text { passage height } \\
& r=\text { radius } \\
& \beta=\text { blade angle with respect to tangent }
\end{aligned}
$$


$t_{b}=$ blade thickness

$N=$ number of blades

The values for the blade pitch are defined by Equation (3-14) which is then used to find the subsonic discharge angle, estimated by Equation (3-15).

$$
\begin{aligned}
& s=2 \pi r / N \\
& \tan \alpha_{3}=\left(r_{3} / r_{t h}\right) \tan \alpha_{o s} \\
& \sin \alpha_{o s}=b_{t h} o /\left(s_{3} b_{3}\right)
\end{aligned}
$$

where,

$o=$ blade to blade width

The optimum incidence angle, $i^{*}$, and the optimum inlet flow angle, $\alpha^{*}$, are defined by,

$$
\begin{aligned}
& i^{*}=\left[3.6 \sqrt{10 t_{b} / L}+\left|\beta_{3}-\beta_{1}\right| / 3.4\right\rfloor \sqrt{L / s_{3}}-\left|\beta_{3}-\beta_{1}\right| / 2 \\
& \alpha^{*}=\beta_{1}-i^{*} \operatorname{sign}\left(\beta_{3}-\beta_{1}\right)
\end{aligned}
$$

The incidence loss coefficient, $Y_{i n c}$, is proportional to the inlet velocity pressure, thus, one uses the fraction of the discharge pressure. Here, $\alpha_{1}$, is the inlet flow angle.

$$
Y_{i n c}=\sin ^{2}\left(\alpha_{1}-\alpha^{*}\right) \frac{P_{t 1}-P_{1}}{P_{t 3}-P_{3}}
$$

The nozzle exit total pressure is defined by $P_{t 3}$, where the total loss coefficient for the nozzle is given by $Y$.

$$
\begin{aligned}
& P_{t 3}=\left(P_{t 1}+Y P_{3}\right) /(1+Y) \\
& Y=Y_{p}+Y_{i n c}=\left(P_{t 1}-P_{t 3}\right) /\left(P_{t 3}-P_{3}\right)
\end{aligned}
$$

\subsubsection{Rotor Analysis}

For a radial inflow turbine, the flow is turned by 90 degrees from the radial inlet to the axial outlet. There are several losses which need to be accounted for and one begins by first defining a few parameters. The first is the average mean surface curvature which is given by, 


$$
\kappa_{m}=\left|\phi_{3}-\phi_{1}\right| / m_{3}
$$

where,

$$
\begin{aligned}
& \varphi=\text { passage curve angle with respect to tangent } \\
& m_{3}=\text { total meridional path length }
\end{aligned}
$$

As was done for the nozzle row, it is important to define the subsonic relative discharge flow angle by using (3-23).

$$
\tan \alpha_{3}^{\prime}=\left(r_{3} / r_{t h}\right) \tan \alpha_{o s}^{\prime}
$$

where,

$$
\sin \alpha_{o s}^{\prime}=b_{t h} o /\left(s_{3} b_{3}\right)
$$

Again Equation (3-14) is used, as was done for the nozzle; however, for the rotor, it is based on the full length blades only.

The optimum (minimal loss) inlet swirl velocity, $C_{1}^{*}$, and absolute flow angle, $\alpha_{1}$, can be calculated using the centrifugal compressor slip factor, $\sigma$.

$$
\begin{aligned}
& C_{\theta 1}^{*}=\sigma\left[U_{1}-C_{m 1} \cot \beta_{1}\right] \\
& \cot \alpha_{1}^{*}=C_{m 1} / C_{\theta 1}^{*}
\end{aligned}
$$

The basic slip factor, $\sigma$, applies for most practical cases, however requires an adjustment if the blade solidity is too low. This situation is evaluated by using the slip factor formula below and the impeller radius ratio, $\varepsilon$.

$$
\begin{aligned}
& \sigma=\left|\sin \phi_{1}\right| \sqrt{\sin \beta_{1}} / N^{0.7} \\
& \varepsilon=r_{3} / r_{1}
\end{aligned}
$$

A simple test is performed to determine if the blade solidity is too low by comparing $\varepsilon$ with $\varepsilon_{\text {lim }}$.

$$
\varepsilon_{\lim }=\left(\sigma-\sigma_{o}\right) /\left(1-\sigma_{o}\right)
$$

where,

$$
\sigma_{o}=\sin \left(19^{\circ}+\beta_{1} / 5\right)
$$

Thus, if $\varepsilon>\varepsilon_{\text {lim }}$, then the slip factor is corrected by using the equation below for $\sigma_{c o r}$

where,

$$
\sigma_{c o r}=\sigma(1-\xi)
$$




$$
\xi=\left[\left(\varepsilon-\varepsilon_{\lim }\right) /\left(1-\varepsilon_{\lim }\right)\right]^{\sqrt{\beta_{1} / 10}}
$$

The rotor, unlike the nozzles, has many more losses which need to be defined. Using the absolute angle calculated earlier, one defines the formula for the incidence loss coefficient, $Y_{\text {inc }}$, below,

$$
Y_{i n c}=\sin ^{2}\left(\alpha_{1}-\alpha_{1}^{*}\right)\left[P_{t 1}^{\prime}-P_{1}\right] /\left[P_{t 3}^{\prime}-P_{3}\right]
$$

The loss coefficient for the blade loading, $Y_{B L}$, and the hub-to-shroud, $Y_{H S}$, loading effects are adapted here from the impeller performance analysis that is used for centrifugal compressors as used by Aungier [13].

$$
Y_{B L}=\frac{1}{24}\left[\frac{\Delta W}{W_{3}}\right]^{2}
$$

where according to Stokes Theorem,

$$
\begin{aligned}
& \Delta W=2 \pi\left|r_{3} C_{\theta 3}-r_{1} C_{\theta 1}\right| /[L(N)] \\
& Y_{H S}=\frac{1}{6}\left[\frac{\kappa_{m} b_{3} W_{2}}{W_{3} \sin \alpha_{3}}\right]^{2}
\end{aligned}
$$

The clearance gap loss coefficient, $Y_{C L}$, is calculated by using the average pressure difference across the blade clearance gap, $\Delta P$, which is estimated from the change in angular momentum in the impeller.

$$
\begin{aligned}
& Y_{C L}=\dot{m}_{C L} \Delta P /\left[\dot{m}\left(P_{t 3}^{\prime}-P_{3}\right)\right] \\
& \Delta P\left[\rho_{\text {ave }}(r b)_{\text {ave }} L N\right]=\dot{m}\left|r_{1} C_{\theta 1}-r_{3} C_{\theta 3}\right|
\end{aligned}
$$

The velocity of the leakage flow, $u_{C L}$, across the clearance gap is calculated by,

$$
u_{C L}=\sqrt{2 \Delta P / \rho_{\text {ave }}}
$$

which then leads to calculating the clearance gap leakage mass flow rate below assuming a gap contraction ration of 0.816 .

$$
\dot{m}_{C L}=0.816 \rho_{a v e} u_{C L} L N \delta_{c}
$$

For this rotating component, the "rothalpy" is conserved through the rotor by,

$$
I=H_{1}+\Delta H_{D F}-\omega r_{1} C_{\theta 1}
$$


Rothalpy in the equation above corrects the inlet total enthalpy for the additional work due to the friction of the rotating disk. Since disk friction parasitic work, $\Delta H_{D F}$, below is calculated for an axial flow turbine, one uses only one-half of the value given, as there is only one side of the disk contributing to this parasitic work.

$$
\Delta H_{D F}=C_{M} \rho \omega^{3} r^{5} / \dot{m}
$$

where the disk torque coefficient $C_{M}$, and the torque, $\tau$, are defined below,

$$
\begin{aligned}
& C_{M}=\tau /\left(\rho \omega^{2} r^{5}\right) \\
& \tau=\dot{m}\left(r_{2} C_{\theta 2}-r_{1} C_{\theta 1}\right)
\end{aligned}
$$

The exit relative enthalpy is defined below, using the variables calculated above.

$$
H_{3}^{\prime}=I+\frac{1}{2} W_{3}^{2}
$$

Now that all of the loss coefficients are defined, one uses the total loss coefficient, $Y$, and calculates the actual exit relative total pressure at the discharge of the rotor in much the same way that one calculates the relative total pressure at the exit of the nozzle.

$$
\begin{aligned}
& Y=Y_{P}+Y_{i n c}+Y_{B L}+Y_{H S}+Y_{C L}+Y_{Q} \\
& P_{t 3}^{\prime}=\left(P_{t 3, i d}^{\prime}+Y P_{3}\right) /(1+Y)
\end{aligned}
$$

Using the conservation of mass, the impeller exit mass balance is calculated by,

$$
\dot{m}=(1-\Delta) 2 \pi r_{3} b_{3} \rho_{3} W_{3} \sin \alpha_{3}^{\prime}
$$

The discharge exit static enthalpy is given by,

$$
h_{3}=H_{3}^{\prime}-\frac{1}{2} W_{3}^{2}
$$

When the impeller discharge relative flow is known from the subsonic solution as presented thus far, the absolute discharge tangential velocity is given by,

$$
C_{\theta 3}=W_{\theta 3}+U_{3}
$$

which leads to the solution for the absolute discharge total enthalpy by,

$$
H_{3}=H_{3}^{\prime}+U_{3} C_{\theta 3}-\frac{1}{2} U_{3}^{2}
$$




\section{Turbomachinery Model Validation}

Partial validation of ANL turbomachinery models has been initiated using the data obtained with the Sandia Brayton Loop [14]. The term "partial validation" is used because the models can only be fully validated through comparison with data obtained using supercritical $\mathrm{CO}_{2}$ under prototypical conditions. The Sandia Brayton Loop cycle has Capstone C-30 turbomachinery with a centrifugal compressor and radial inflow turbine. Since data obtained using gaseous $\mathrm{CO}_{2}$ was not available at the time this report was prepared, nitrogen properties have been programmed into the turbomachinery codes. Even though nitrogen properties could be calculated fairly accurately under the ideal gas approximation, the turbomachinery codes were developed for a real fluid which may have significant property variations with pressure and temperature. Therefore, the nitrogen properties are being calculated and used in the same way as $\mathrm{CO}_{2}$ properties for the analysis; that is, highly detailed nitrogen properties are used without any deal gas simplifications.

\subsection{Compressor Performance Model Validation}

The experiment data for a centrifugal compressor with nitrogen as the working fluid have been used for validation of the compressor performance model. The available measured data includes:

- Rotational speed,

- Inlet total temperature,

- Inlet total pressure,

- Outlet total pressure.

The compressor performance model requires input data sufficient to describe the compressor design. (Usually, this data is obtained from the compressor design code.) Table 4-1 shows the list of required parameters along with the values assumed in the model. It should be mentioned here that the exact compressor design parameters are considered to be proprietary information of Capstone and are not available to SNL. An estimation of the parameters in Table 4-1 has been made by SNL based on photographs taken during loop construction as well as similar photographs taken by NASA. Therefore, the values in Table 4-1 could not be considered to represent the exact compressor design but only an approximation. At the same time, it was noticed that the photographs from which estimates were made are sometimes inconsistent. For example, the ratio of an impeller discharge radius to the impeller inlet hub radius is different for different set of pictures, raising a suspicion that those photographs do not show the same compressor. Moreover, some of the parameters in Table 4-1 could not be measured from the photographs, such as surface roughness and clearance gap at working conditions. It is believed that some values of these parameters were simply assumed by SNL. Besides, some parameters, like blade length, were not provided by SNL and were estimated from one of the photographs. 
Table 4-1. Compressor Design Parameters for the Performance Model

\begin{tabular}{|c|c|c|}
\hline Parameter & Value & Comments \\
\hline \multicolumn{3}{|c|}{ General } \\
\hline Number of stages & 1 & \\
\hline IGV angle, deg & 0 & \\
\hline Blade surface roughness, $\mu \mathrm{m}$ & 4.7 & \\
\hline Number of labyrinth seals on impeller tip & 0 & 0-unshrouded \\
\hline \multicolumn{3}{|c|}{ Impeller } \\
\hline Impeller hub radius at inlet, $\mathrm{mm}$ & 12.83 & \\
\hline Impeller tip radius at inlet, $\mathrm{mm}$ & 27.95 & \\
\hline Impeller inlet blade angle, deg & -60.8 & with respect to axial direction \\
\hline Impeller discharge blade angle, deg & -34 & with respect to radial direction \\
\hline Impeller discharge radius, $\mathrm{mm}$ & 50.81 & \\
\hline Blade height at discharge, $\mathrm{mm}$ & 7.0 & \\
\hline Number of blades, full/splitter & $9 / 9$ & \\
\hline $\begin{array}{l}\text { Splitter blade length, fraction of full blade } \\
\text { length }\end{array}$ & 0.7 & \\
\hline Blade thickness, mm & 1.8 & \\
\hline Blade length, $\mathrm{mm}$ & 4052 & \\
\hline Tip (shroud) clearance-to-radius ratio & 0.005 & \\
\hline \multicolumn{3}{|c|}{ Diffuser (nozzle) } \\
\hline Number of diffuser blades & 19 & 0 -vaneless diffuser \\
\hline Diffuser inner radius, $\mathrm{mm}$ & 54.86 & \\
\hline Diffuser outer radius, $\mathrm{mm}$ & 84.22 & \\
\hline Diffuser inlet blade angle, deg & 77.0 & with respect to radial direction \\
\hline Diffuser discharge blade angle, deg & 39.0 & with respect to radial direction \\
\hline Blade height at inlet, $\mathrm{mm}$ & 3.99 & \\
\hline Blade height at discharge, $\mathrm{mm}$ & 3.99 & \\
\hline Diffuser blade thickness at inlet, $\mathrm{mm}$ & 0.8 & \\
\hline Diffuser blade thickness at discharge, $\mathrm{mm}$ & 15.6 & \\
\hline Blade length, $\mathrm{mm}$ & 52.74 & \\
\hline
\end{tabular}

The comparison of the model results with the experiment data was carried out for the tests for pure nitrogen for $700 \mathrm{~K}$ and $870 \mathrm{~K}$ turbine inlet temperature (Tables 3-1 and $3-5$ of [14]). The summary of the test data relative to the compressor is reproduced in Table 4-2. Unfortunately, neither the compressor outlet temperature nor the compressor efficiency are reported, so the efficiency comparison could not be made. The inlet conditions from Table 4-2 were supplied to the compressor performance model. Figure 4-1 shows the comparison of the results from the model calculations with the experimental data. 
Table 4-2. Compressor Experiment Data for Pure Nitrogen

\begin{tabular}{|c|c|c|c|c|c|}
\hline $\begin{array}{c}\text { Turbine } \\
\text { inlet } \\
\text { temperature }\end{array}$ & $\begin{array}{c}\text { Rotational } \\
\text { speed }\end{array}$ & $\begin{array}{l}\text { Mass flow } \\
\text { rate }\end{array}$ & $\begin{array}{l}\text { Compressor } \\
\text { inlet total } \\
\text { temperature }\end{array}$ & $\begin{array}{c}\text { Compressor } \\
\text { inlet total } \\
\text { pressure }\end{array}$ & $\begin{array}{c}\text { Compressor } \\
\text { outlet total } \\
\text { pressure }\end{array}$ \\
\hline $\mathbf{K}$ & krpm & $\mathrm{kg} / \mathrm{s}$ & $\mathbf{K}$ & $\mathbf{k P a}$ & $\mathbf{k P a}$ \\
\hline \multirow{4}{*}{700} & 55 & 0.17 & \multirow{4}{*}{290} & 127 & 204 \\
\hline & 60 & 0.19 & & 122.5 & 213 \\
\hline & 65 & 0.205 & & 117.5 & 222.5 \\
\hline & 70 & 0.22 & & 112.5 & 229 \\
\hline & & & & & \\
\hline \multirow{10}{*}{870} & 25 & 0.05 & \multirow{10}{*}{290} & 129 & 145.5 \\
\hline & 30 & 0.065 & & 127.5 & 150 \\
\hline & 40 & 0.1 & & 124 & 163 \\
\hline & 50 & 0.13 & & 118.7 & 178 \\
\hline & 60 & 0.158 & & 112 & 196 \\
\hline & 70 & 0.185 & & 104 & 215 \\
\hline & 75 & 0.202 & & 100 & 225.5 \\
\hline & 80 & 0.215 & & 96 & 236 \\
\hline & 85 & 0.225 & & 92 & 246.3 \\
\hline & 90 & 0.238 & & 88 & 257 \\
\hline
\end{tabular}

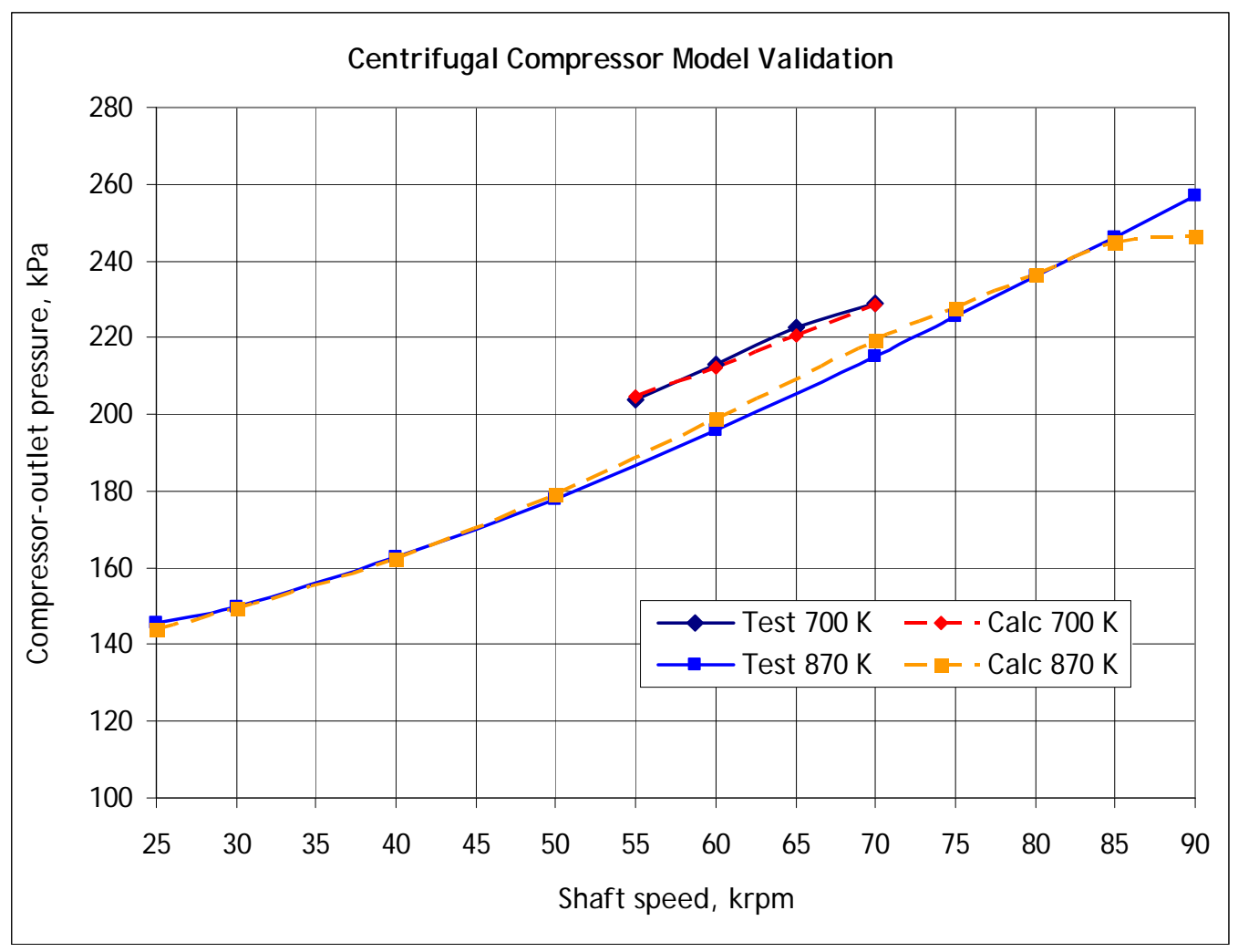

Figure 4-1. Comparison of Compressor Experiment Data with Model Calculations. 
The model predictions in Figure 4-1 are, in general, close to the data. The only significant difference is at the last point for the $870 \mathrm{~K}$ line. This difference could be explained by the uncertainty in the measured data. For example, the uncertainty in flow rate is reported as $0.01 \mathrm{~kg} / \mathrm{s}$ [14]. Therefore, the actual flow rate may be different from that reported in Table 4-2. To estimate the effect of this uncertainty, the model was rerun with the same parameters except that the flow rate in the last line was changed from 0.238 to $0.228 \mathrm{~kg} / \mathrm{s}$ (i.e., within the uncertainty range). Figure $4-2$ shows how this small change in flow rate affects the results (compared to Figure 4-1). The model prediction now agrees very well with the experiment results.

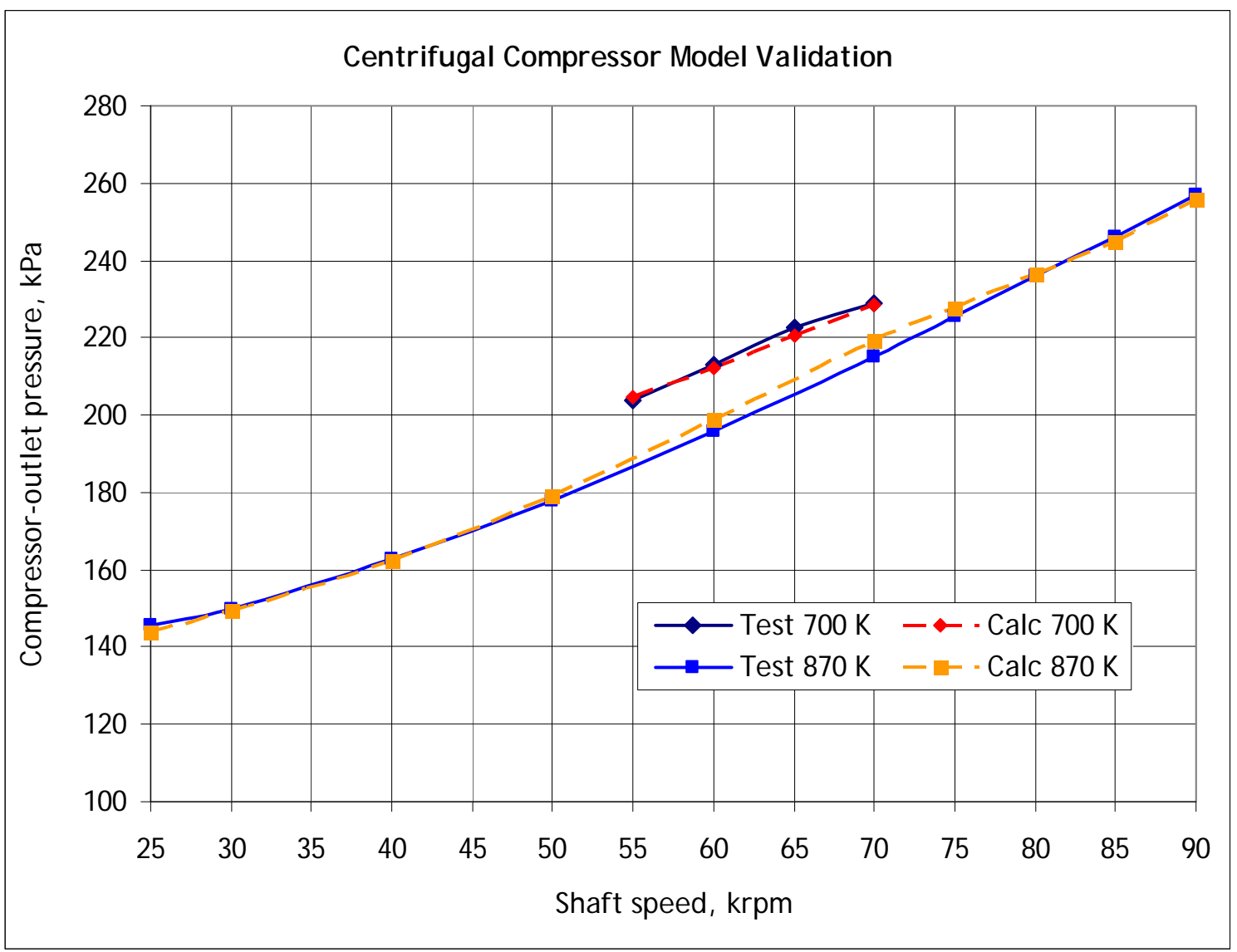

Figure 4-2. Comparison of Compressor Experiment Data with Model Calculations with Flow Rate Modified within Uncertainty Range. 


\subsection{Summary of Compressor Model Validation}

Even though the exact compressor design parameters were not available, and some parameters had to be assumed, the calculated results in terms of outlet pressure show good agreement with measured data. It is also demonstrated that the experiment uncertainty can have a significant effect on the degree of agreement between calculation and experiment. Within the uncertainty range for flow rate only, very close agreement can be achieved between calculations and experiment. Therefore, it is concluded that no model modifications are required at this time. Compressor efficiency or compressor outlet temperature data would provide significant additional information for still more meaningful model validation.

\subsection{Turbine Model Calculations}

The results of radial inflow turbine model calculations are shown in Table 4-3. Many of the inputs for the model were taken from Reference [14]; however, it is necessary to estimate a few input values for which information was not provided. Unfortunately, it is not known how reliable these estimates are for the Capstone C-30 micro turbine. Figure 4-3 through Figure 4-10 show the calculated behavior of the nitrogen gas inside the turbine at different pressures for varying rotor speeds. It is observed that the turbine is calculated to have a maximum efficiency of about $75 \%$ which is close to what is expected [13]. A next step would be to compare the model calculations with test data and to vary the values of the estimated inputs to attempt to better determine appropriate values for the Capstone C-30 turbomachinery. 
Table 4-3. Radial Inflow Turbine Model Calculations for Pure Nitrogen at Turbine Inlet Temperature (TIT) of $700 \mathrm{~K}$

\begin{tabular}{|c|c|c|c|c|}
\hline \multicolumn{1}{|c}{ N2 } & Temperature In & $\mathbf{7 0 0}[\mathbf{K}]$ & & \\
\hline krpm & Mass Flow Rate & Pressure In [kPa] & Pressure Out [kPa] & Pressure Ratio \\
\hline 55 & 0.1563 & 199.0 & 126.4 & 1.57439 \\
60 & 0.1563 & 199.0 & 123.3 & 1.61413 \\
65 & 0.1563 & 199.0 & 120.2 & 1.65524 \\
70 & 0.1563 & 199.0 & 117.0 & 1.70094 \\
\hline 55 & 0.1631 & 207.5 & 126.2 & 1.57646 \\
60 & 0.1631 & 207.5 & 123.1 & 1.61603 \\
65 & 0.1631 & 207.5 & 120.1 & 1.65707 \\
70 & 0.1631 & 207.5 & 116.8 & 1.70310 \\
\hline 55 & 0.1701 & 216.5 & 126.3 & 1.57528 \\
60 & 0.1701 & 216.5 & 123.2 & 1.61496 \\
65 & 0.1701 & 216.5 & 120.2 & 1.65605 \\
70 & 0.1701 & 216.5 & 116.9 & 1.70189 \\
\hline 55 & 0.1773 & 225.5 & 126.2 & 1.57726 \\
60 & 0.1773 & 225.5 & 123.1 & 1.61678 \\
65 & 0.1773 & 225.5 & 120.0 & 1.65781 \\
70 & 0.1773 & 225.5 & 116.8 & 1.70395 \\
\hline
\end{tabular}




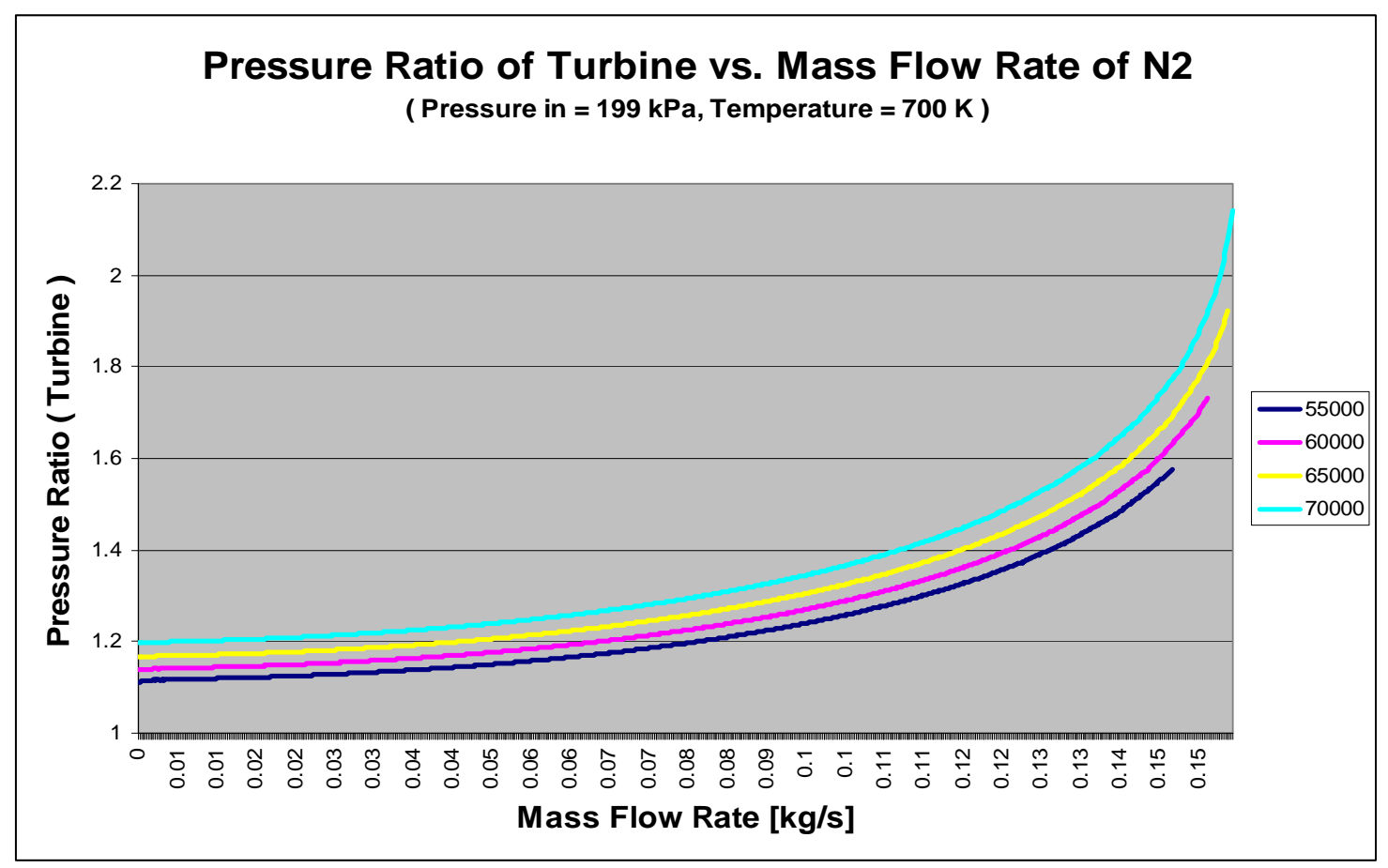

Figure 4-3. Prediction of Radial Inflow Turbine Model for Pressure Ratio versus Mass Flow Rate for Pure Nitrogen at Turbine Inlet Pressure (TIP) of $199 \mathrm{kPa}$ and Turbine Inlet Temperature (TIT) of $700 \mathrm{~K}$.

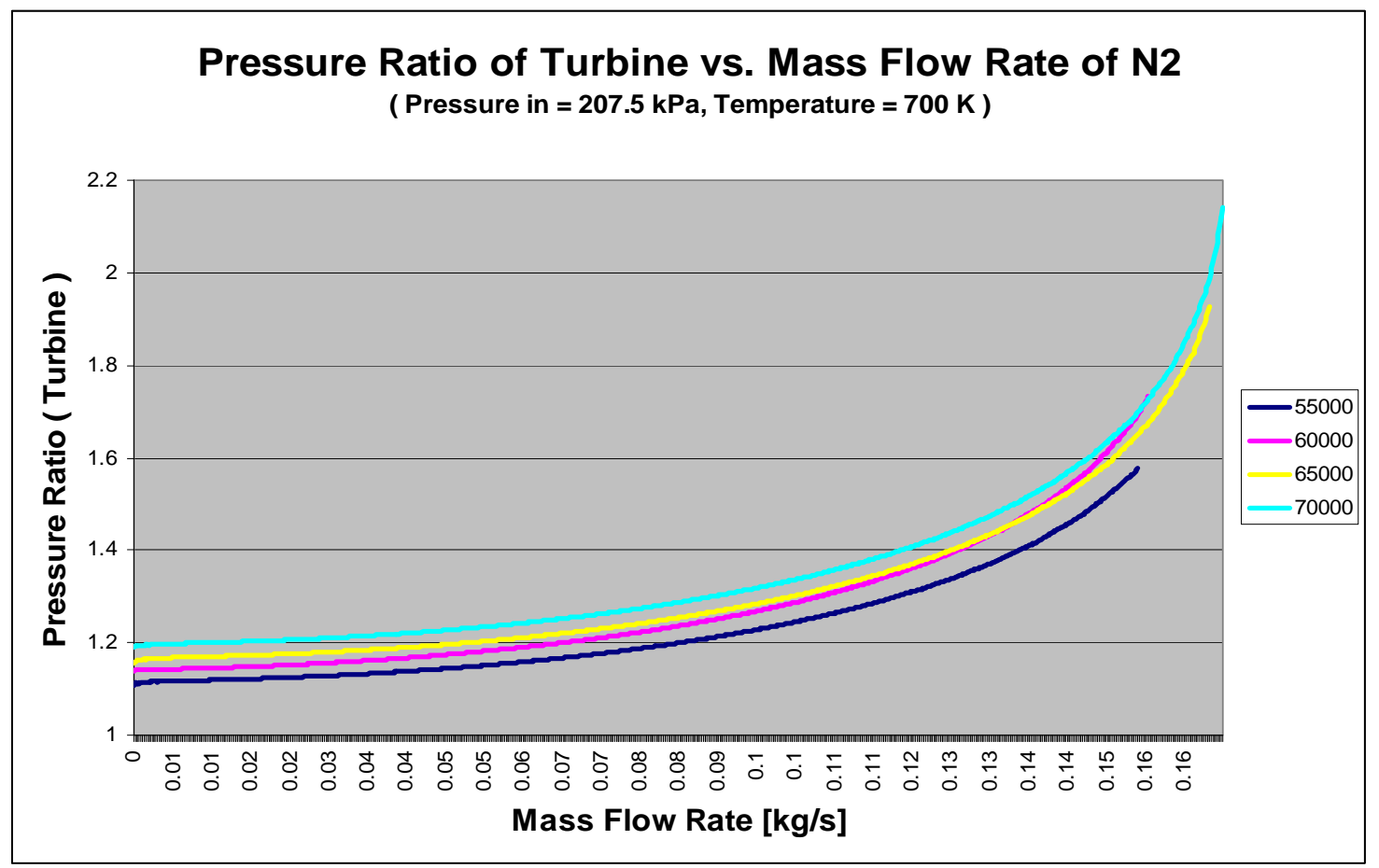

Figure 4-4. Prediction of Radial Inflow Turbine Model for Pressure Ratio versus Mass Flow Rate for Pure Nitrogen at Turbine Inlet Pressure (TIP) of $207.5 \mathrm{kPa}$ and Turbine Inlet Temperature (TIT) of $700 \mathrm{~K}$. 


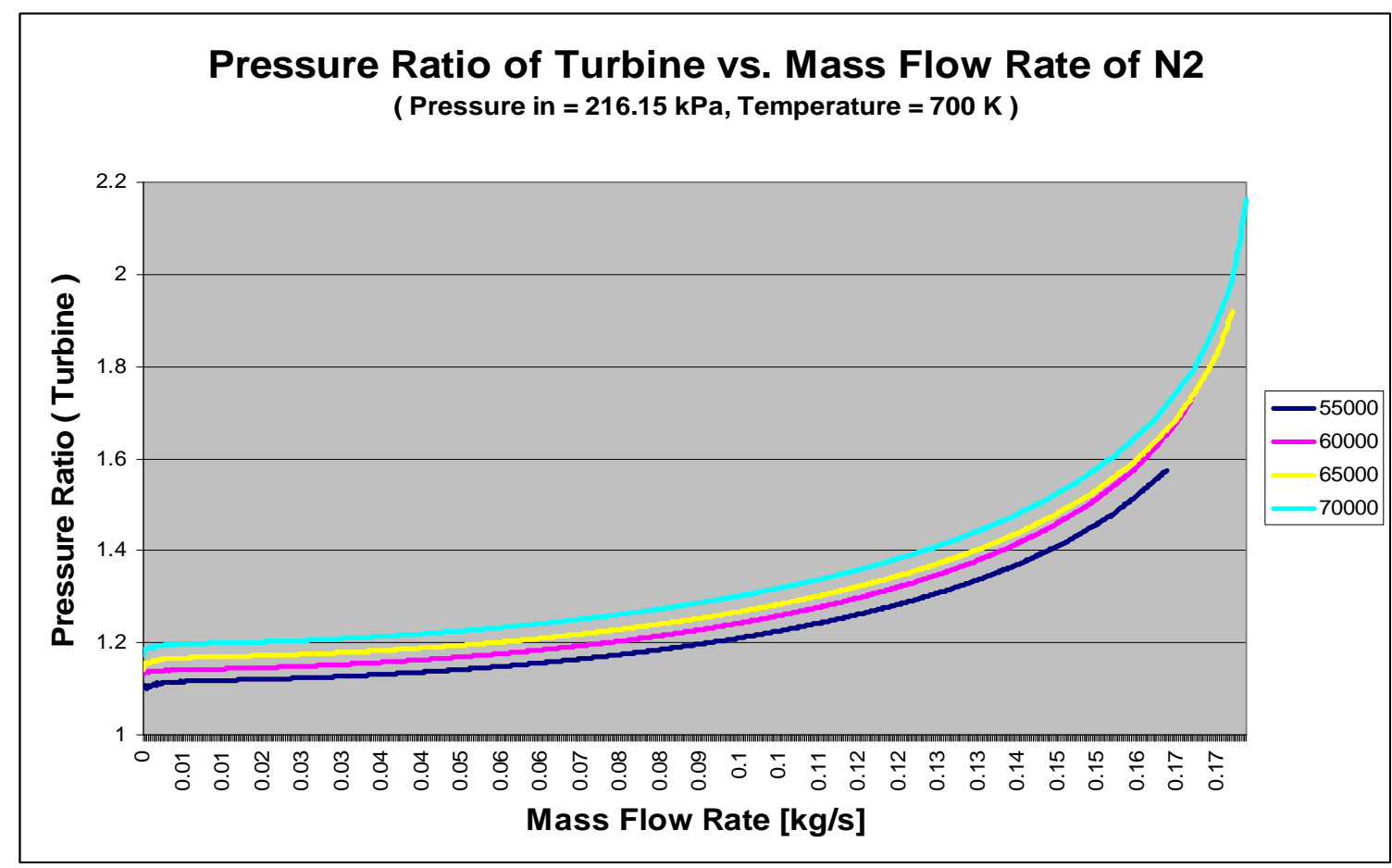

Figure 4-5. Prediction of Radial Inflow Turbine Model for Pressure Ratio versus Mass Flow Rate for Pure Nitrogen at Turbine Inlet Pressure (TIP) of $216.5 \mathrm{kPa}$ and Turbine Inlet Temperature (TIT) of $700 \mathrm{~K}$.

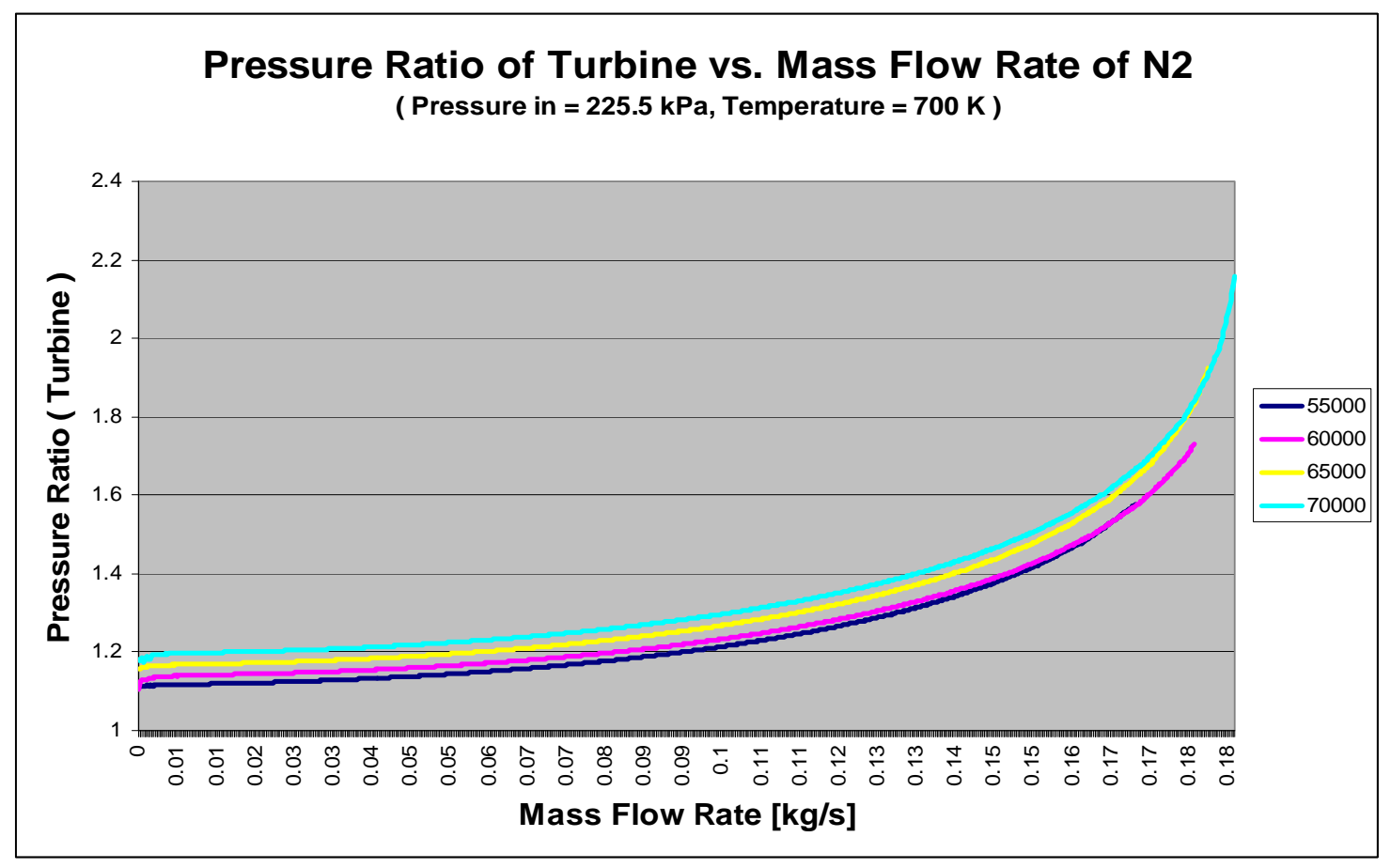

Figure 4-6. Prediction of Radial Inflow Turbine Model for Pressure Ratio versus Mass Flow Rate for Pure Nitrogen at Turbine Inlet Pressure (TIP) of $225.5 \mathrm{kPa}$ and Turbine Inlet Temperature (TIT) of $700 \mathrm{~K}$. 


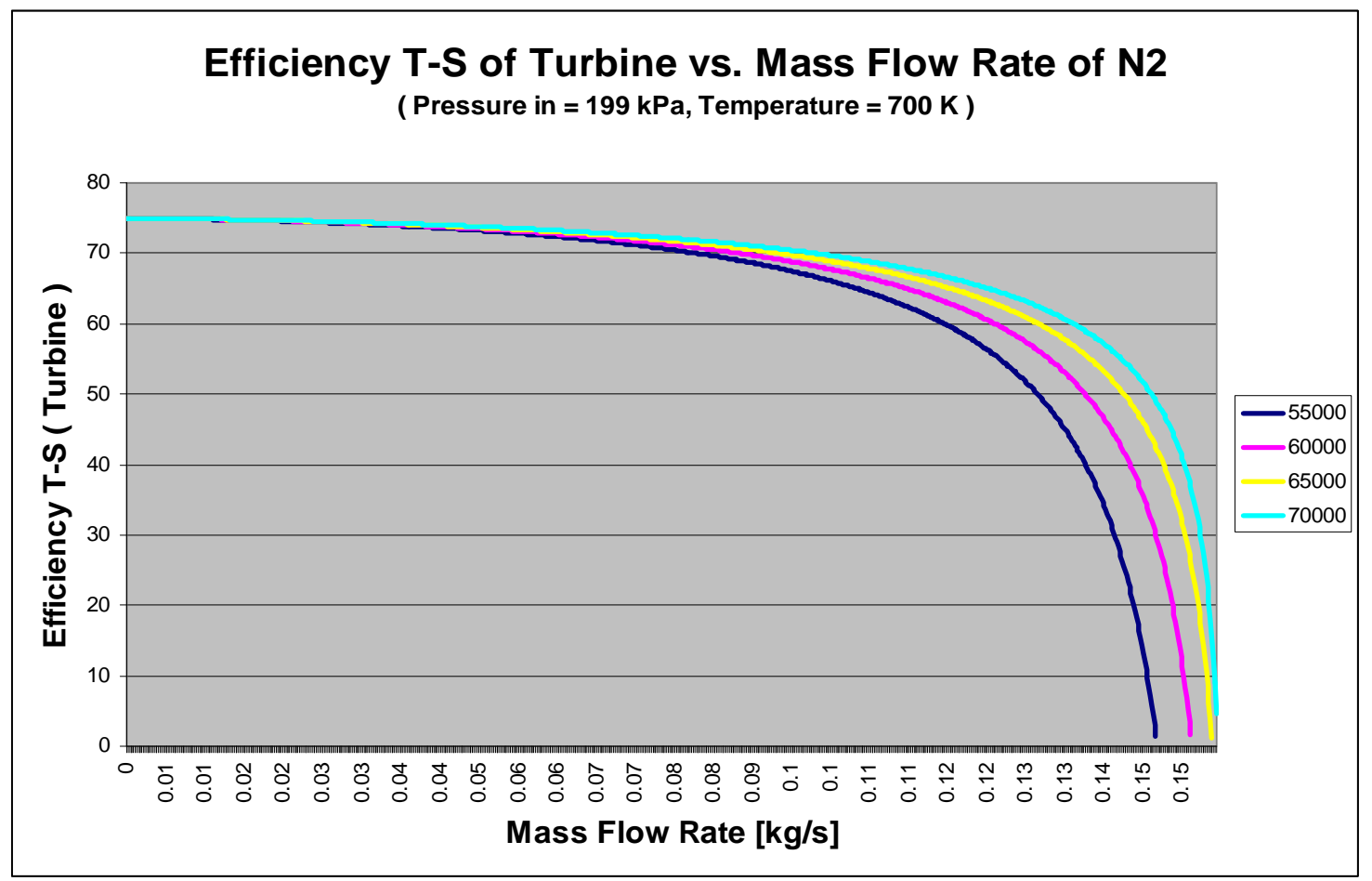

Figure 4-7. Prediction of Radial Inflow Turbine Model for Efficiency (Total-toStatic) versus Mass Flow Rate for Pure Nitrogen at Turbine Inlet Pressure (TIP) of $199 \mathrm{kPa}$ and Turbine Inlet Temperature (TIT) of $700 \mathrm{~K}$.

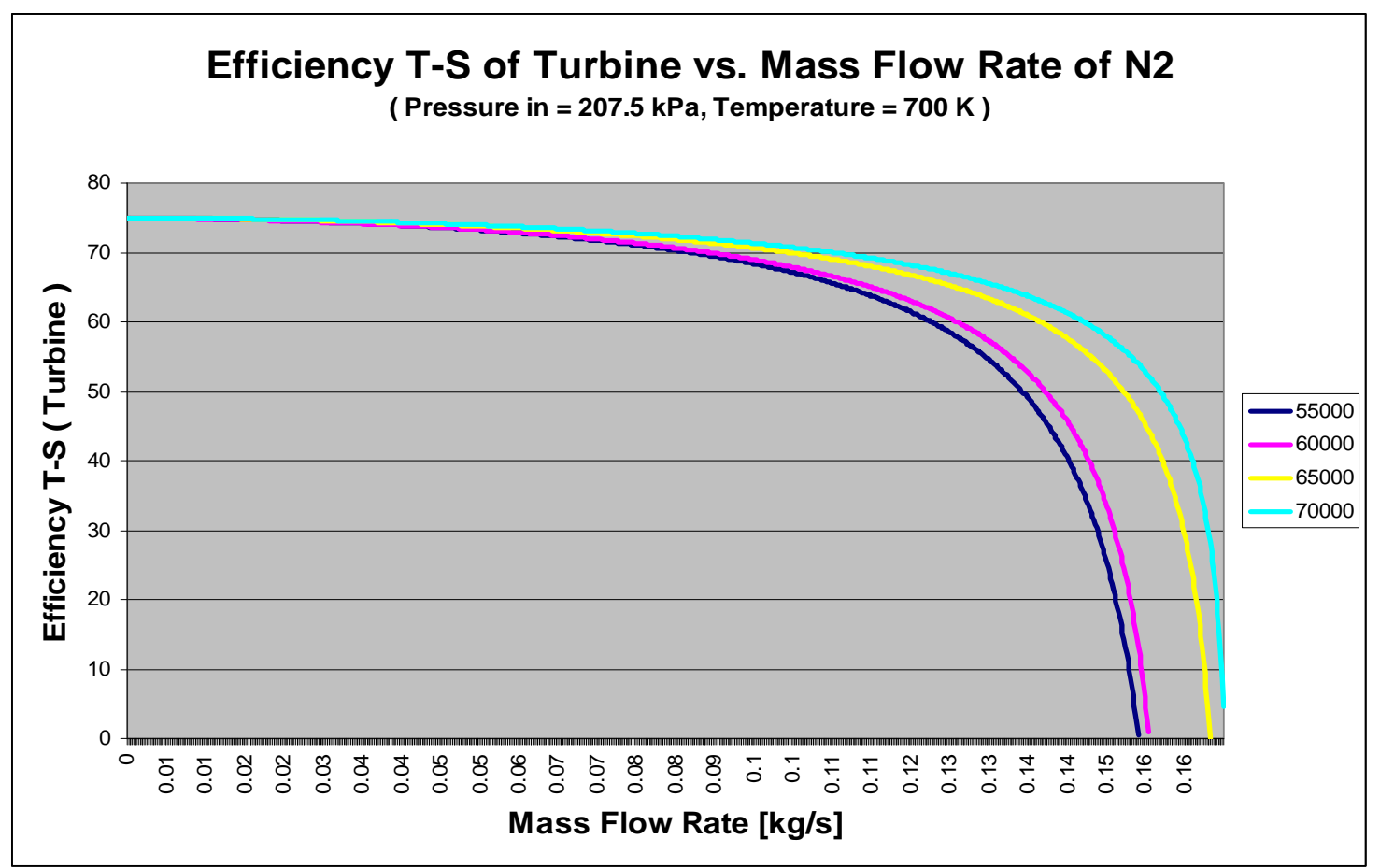

Figure 4-8. Prediction of Radial Inflow Turbine Model for Efficiency (Total-toStatic) versus Mass Flow Rate for Pure Nitrogen at Turbine Inlet Pressure (TIP) of $207.5 \mathrm{kPa}$ and Turbine Inlet Temperature (TIT) of $700 \mathrm{~K}$. 


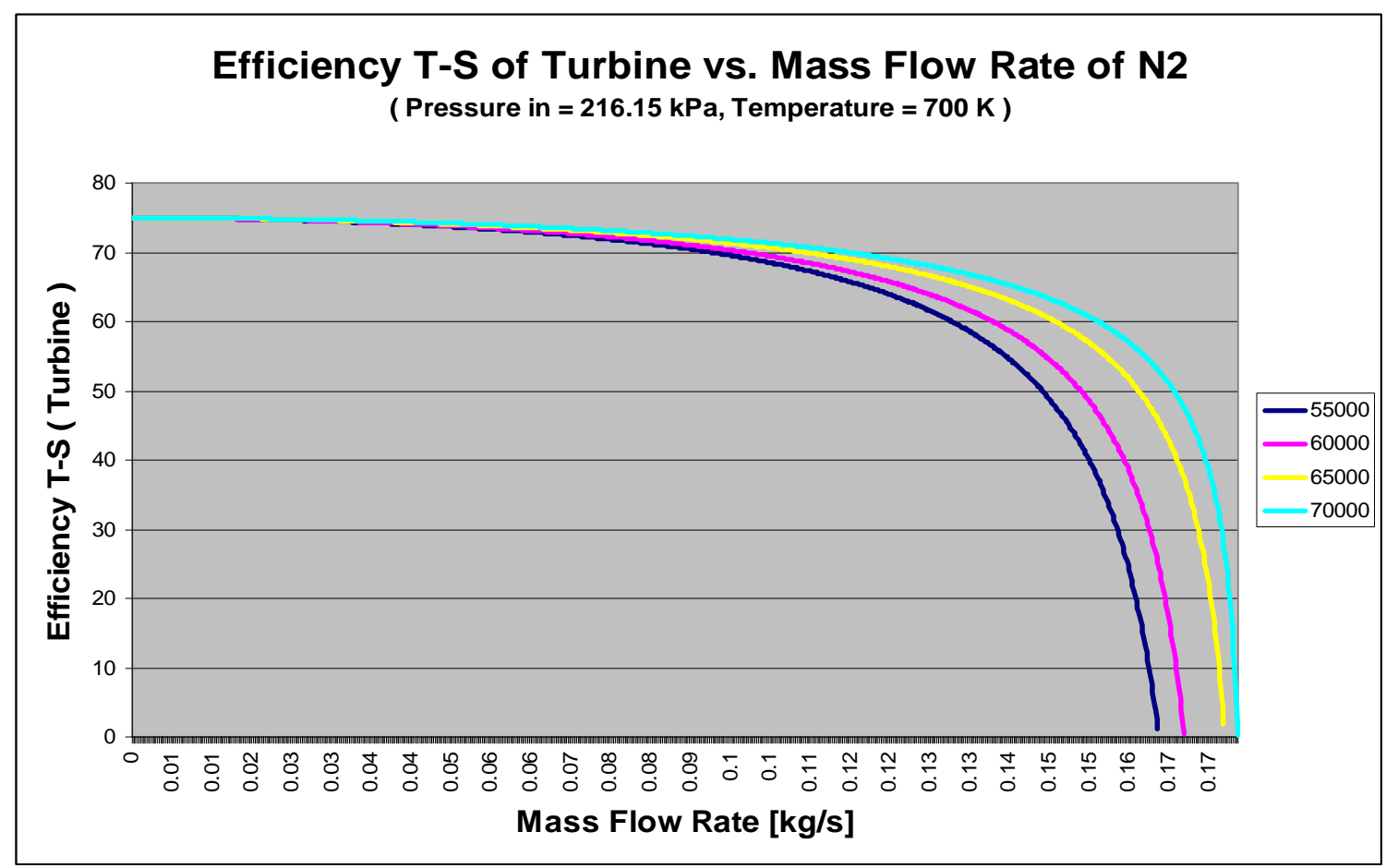

Figure 4-9. Prediction of Radial Inflow Turbine Model for Efficiency (Total-toStatic) versus Mass Flow Rate for Pure Nitrogen at Turbine Inlet Pressure (TIP) of $216.5 \mathrm{kPa}$ and Turbine Inlet Temperature (TIT) of $700 \mathrm{~K}$.

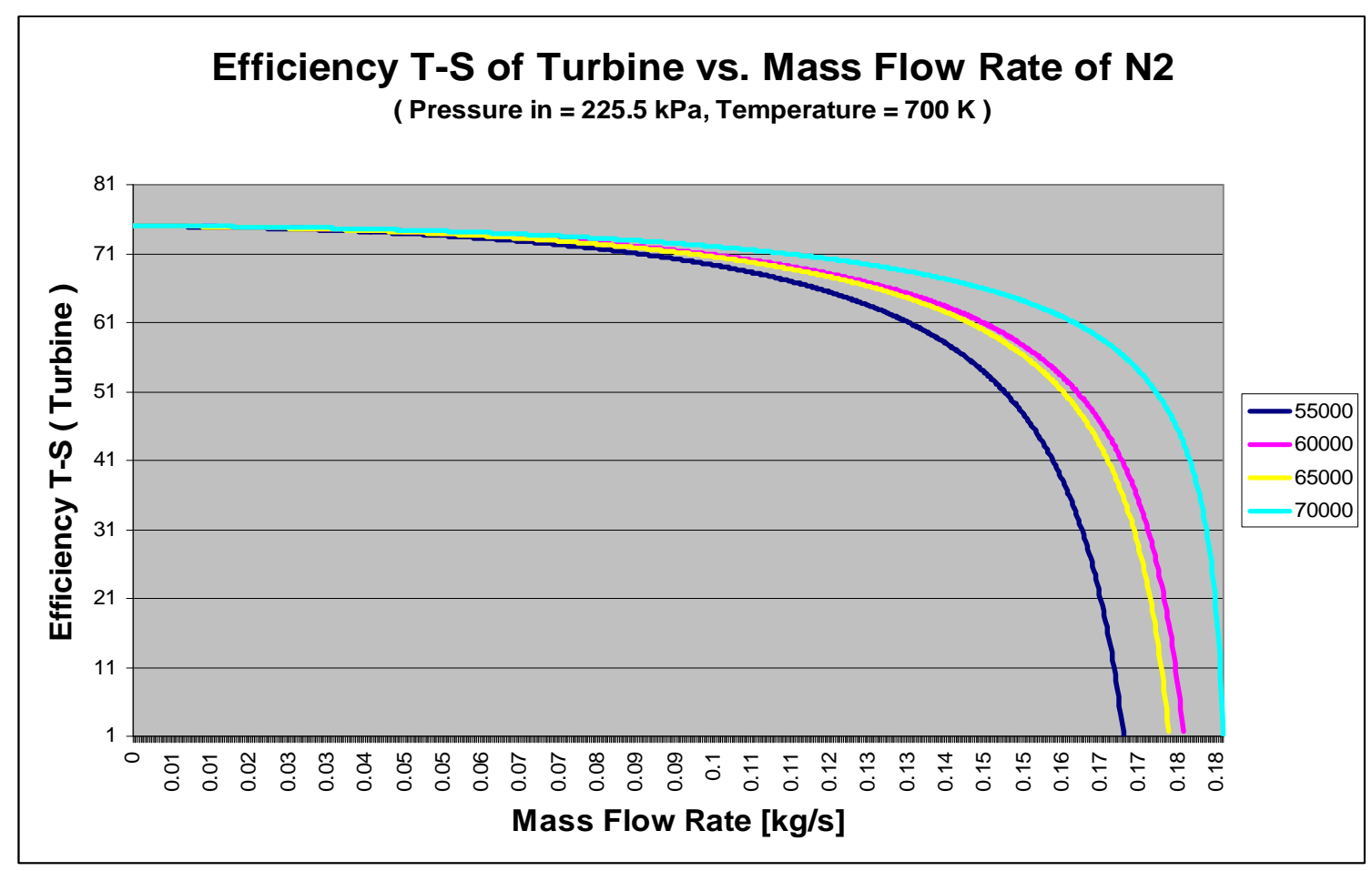

Figure 4-10. Prediction of Radial Inflow Turbine Model for Efficiency (Total-toStatic) versus Mass Flow Rate for Pure Nitrogen at Turbine Inlet Pressure (TIP) of $225.5 \mathrm{kPa}$ and Turbine Inlet Temperature (TIT) of $700 \mathrm{~K}$. 


\section{Publications and Other Activities}

The following summary and peer-reviewed conference papers have been published during this fiscal year:

- A. Moisseytsev and J. J. Sienicki, "Control of Supercritical $\mathrm{CO}_{2}$ Brayton Cycle for LFR Autonomous Load Following," Transactions of the American Nuclear Society, Vol. 93, p. 342, American Nuclear Society 2005 Winter Meeting, Washington, DC, November 13-17, 2005.

- A. Moisseytsev and J. J. Sienicki, "Automatic Control Strategy Development for the Supercritical $\mathrm{CO}_{2}$ Brayton Cycle for LFR Autonomous Load Following," Proceedings of ICAPP 06, 2006 International Congress on Advances in Nuclear Power Plants, Reno, NV USA, June 4-8, 2006, Paper 6074.

- A. Moisseytsev and J. J. Sienicki, "Transient Accident Analysis of a Supercritical Carbon Dioxide Brayton Cycle Energy Converter Coupled to an Autonomous Lead-Cooled Fast Reactor," Proceedings of the 14th International Conference on Nuclear Engineering, ICONE-14, Miami, FL, July 17-20, 2006.

A. Moisseytsev attended the "Centrifugal Compressor Operations for 21st Century Users" short course presented by the Texas A\&M University Turbomachinery Laboratory in San Diego, CA, February 20-24, 2006. The course covered a wide range of the centrifugal compressor issues from design to operating aspects. The course was very helpful to Moisseytsev in developing centrifugal compressor design and performance models.

J. J. Sienicki visited Heatric, a subsidiary of Meggitt (UK), Ltd. in Holton Heath, United Kingdom on July 11, 2006. He held discussions with their specialists and management on their Printed Circuit Heat Exchanger ${ }^{\mathrm{TM}}\left(\mathrm{PCHE}^{\mathrm{TM}}\right)$ and Formed Plate Heat Exchanger (FPHE) products which are envisioned for use with the $\mathrm{S}-\mathrm{CO}_{2}$ Brayton Cycle. He also toured their Fabrication Facilities Factory.

\section{Acknowledgements}

Argonne National Laboratory's work was supported by the U. S. Department of Energy Generation IV Nuclear Energy Systems Initiative. The authors are grateful to Dr. Paul S. Pickard (Sandia National Laboratories), the Generation IV National Technical Director for Energy Conversion, and to Dr. Carl Sink, the U.S. DOE Program Manager for Energy Conversion, for their continued support for development and validation of the $\mathrm{S}-\mathrm{CO}_{2}$ Brayton Cycle Plant Dynamics Computer Code. The three-dimensional drawing of a centrifugal compressor was started by Anna Nikiforova (MIT). The authors are also indebted to many individuals for their helpful advice during the course of this work including Drs. James E. Cahalan, David C. Wade, George Klopp, and Roald A. Wigeland (ANL/NE), P. Hejzlar, M. Driscoll, and Y. Wang (MIT), S. Wright (SNL), as well as Kenneth Nichols and Robert Fuller (Barber-Nichols, Inc.). 


\section{References}

1. Moisseytsev, A. and Sienicki J.J., "Control System Options and Strategies for Supercritical $\mathrm{CO}_{2}$ Cycles," Deliverable Report, WBS No. 1.10.03, August 1, 2005.

2. Hewitt, G.F., Shires, G.L., and Polezhaev, Y.V. International Encyclopedia of Heat and Mass Transfer, CRC Press LLC, 1997.

3. Yan, X., "Dynamic Analysis and Control System Design for an Advanced Nuclear Gas Turbine Power Plant," Ph.D. Dissertation, Massachusetts Institute of Technology, 1990.

4. Tong, L.S. and Weisman, J. Thermal Analysis of Pressurized Water Reactors, American Nuclear Society, 1979.

5. Aungier, R.H., Axial-Flow Compressors: a Strategy for Aerodynamic Design and Analysis, ASME Press, New York, 2003.

6. Wang, Y., Guenette G.R., Hejzlar P., and Driscoll M.J. “Aerodynamic Design of Turbomachinery for 300 MWe Supercritical Carbon Dioxide Brayton Power Conversion System," MIT-GFR-022, Massachusetts Institute of Technology, 2005.

7. Kacker, S.C. and Okapuu, U. "A Mean Line Prediction Method for Axial Flow Turbine Efficiency," Journal of Engineering for Power, Vol. 104, pp. 111-119, 1982.

8. Ainley, D.G. and Mathieson, G.C.R. "A Method of Performance Estimation for Axial-Flow Turbine,” Aeronautical Research Council, R\&M 2974, 1957.

9. Beer, F.P. and Johnston, E.R. Jr., Vector Mechanics for Engineers. Statics and Dynamics, McGraw-Hill, Inc., 1977.

10. Moisseytsev, A. and Sienicki, J. J., "Control of Supercritical $\mathrm{CO}_{2}$ Brayton Cycle for LFR Autonomous Load Following," Transactions of the American Nuclear Society, Vol. 93, p. 342, American Nuclear Society 2005 Winter Meeting, Washington, DC, November 13-17, 2005.

11. Nichols, K. E. and Fuller, R. L., "Review of Turbomachinery for a Supercritical $\mathrm{CO}_{2}$ Power Cycle", Barber-Nichols Inc., September 7, 2005.

12. Aungier, R.H., Centrifugal Compressors: a Strategy for Aerodynamic Design and Analysis, ASME Press, New York, 2000.

13. Aungier, R.H., Turbine Aerodynamics: Axial-Flow and Radial Inflow Turbine Design and Analysis, ASME Press, New York, 2006. 
14. Wright, S. A., Brown, N.R., Pickard, P.S., March, A.J., and Sanchez, T.J., "Analysis of the Dynamic Response of a $30 \mathrm{kWe}$ Closed Brayton Cycle Power Conversion System" Sandia Report (Draft), SAND2006-XXX, June 2006. 


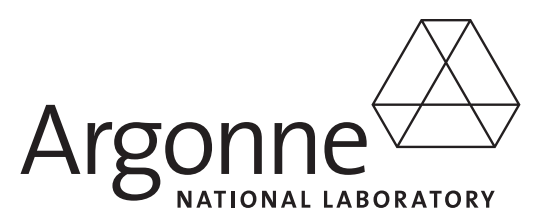

Nuclear Engineering Division

Argonne National Laboratory

9700 South Cass Avenue, Bldg. 208

Argonne, IL 60439-4842

www.anl.gov 